\title{
Scattering Resonances for Polyhedral Obstacles
}

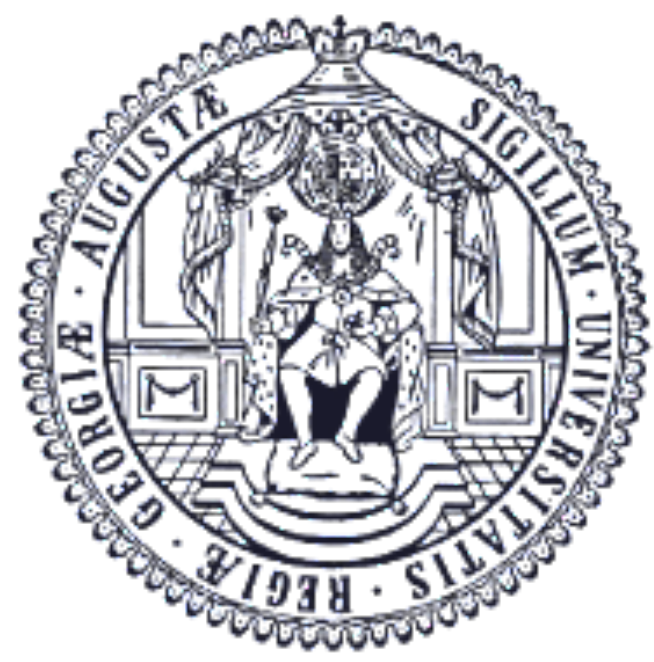

\section{Dissertation}

zur Erlangung des mathematisch-naturwissenschaftlichen Doktorgrades

"Doctor rerum naturalium" der Georg-August-Universität Göttingen

im Promotionsprogramm SMS

der Georg-August-Universität Göttingen (GAUSS)

vorgelegt von

Martin Lippl

geboren in Heide

Göttingen, 2016 

Betreuungsausschuss:

Prof. Dr. Ingo F. Witt, Institute for Mathematics (Referent)

Prof. Dr. Dorothea Bahns, Institute for Mathematics (Korreferentin)

Mitglieder der Prüfungskomission:

Prof. Dr. Ingo F. Witt, Institute for Mathematics (Referent)

Prof. Dr. Dorothea Bahns, Institute for Mathematics (Korreferentin)

Weitere Mitglieder der Prüfungskomission:

Prof. Dr. Preda V. Mihăilescu, Institute for Mathematics

Prof. Dr. Gert Lube, Institute of Numerical and Applied Mathematics

Prof. Dr. Plonka-Hoch, Institute of Numerical and Applied Mathematics

Prof. Dr. David Russel Luke, Institute of Numerical and Applied Mathematics

Tag der mündlichen Prüfung: 30. August 2016 

To IERR 

There was an owl liv'd in an oak

The more he heard, the less he spoke

The less he spoke, the more he heard.

$O$, if men were all like that wise bird!

UNKNOWN AUTHOR

Published in Punch Vol. LXVIII, April 10, 1875 



\begin{abstract}
This thesis deals with the generalization of two dimensional obstacle scattering theory to polygonally bounded obstacles. Our main objective is to derive an upper bound for the counting function of the scattering poles. The counting function counts the number of scattering poles on the analytical continuation of the scattering matrix which, in even dimensions, lives on the Riemann surface of the logarithm.

The starting points for our investigation are P. D. Lax and R. S. Phillips' formulation of scattering theory in an even number of spatial dimensions and $\mathrm{R}$. Melrose's polynomial bound for the counting function in an odd number of spatial dimensions. We restrict ourselves to polygonally bounded obstacles with edges of $C^{\infty}$-type. The key ingredient is the application of Mellin pseudodifferential methods. In the course of this work, we analyse the mapping behaviour of the single and double layer potentials and their traces on the boundary, the Calerón projectors. As a by-product, we derive modified jump conditions for the layer potentials.
\end{abstract}

\title{
Zusammenfassung
}

Diese Dissertation beschäftigt sich mit der Verallgemeinerung der zweidimensionalen Streutheorie auf polygonal berandete Objekte. Ziel dieser Arbeit ist die Herleitung einer oberen Abschätzung für die sogenannte Zählfunktion. Diese Zählfunktion zählt die Anzahl der Streupole, die innerhalb eines Kreises mit dem Radius $r>0$ enthalten sind.

Die Ausgangspunkte unserer Untersuchung sind die geradedimensionale Formulierung der Streutheorie nach P. D. Lax und R. D. Phillips und der Beweis einer oberen Abschätzung für die Zählfunktion von R. Melrose. Neu ist die Einführung des sogenannten Mellin-Kalküls aus der singulären Analysis. Dieses ersetzt den konventionellen Kalkül der pseudodifferentiellen Operatoren. Im Verlaufe der Arbeit untersuchen wir außerdem das Abbildungsverhalten der Einfach- und Doppelschichtpotentiale und Ihrer Spur auf dem Polygonalen Rand des Streuobjekts. Als ein Nebenprodukt leiten wir modifizierte Sprungrelationen her. 


\section{Contents}

1 Introduction 1

2 Fundamentals of Scattering Theory 9

2.1 Abstract Scattering Theory . . . . . . . . . . . . . . . . 9

2.2 Obstacle Scattering . . . . . . . . . . . . . . . . . 11

2.2.1 Formulation of the Problem . . . . . . . . . . . 11

2.2.2 Spectral Theory . . . . . . . . . . . . . . . 12

2.2.3 The Scattering Matrix . . . . . . . . . . . . 13

3 Fundamentals of Mellin Calculus $\quad \mathbf{1 5}$

3.1 Curvilinear Polygon in Two Dimensions . . . . . . . . . . . . . 15

3.2 Weighted Spaces with Asymptotics . . . . . . . . . . . . 18

3.2.1 Sobolev Spaces with Asymptotics in a Cone . . . . . . . . 18

3.2.2 Sobolev Spaces on the Boundary . . . . . . . . . . . . . 22

3.2.3 Sobolev Spaces in the Exterior Domain . . . . . . . . . . 24

3.3 The Mellin Calculus on $\mathbb{R}_{+} \ldots \ldots . \ldots . \ldots . \ldots 25$

3.3.1 Mellin Pseudodifferential Operators ......... . 25

3.3.2 Conormal Symbols and Ellipticity on $\mathbb{R}_{+} \ldots \ldots 27$

4 Boutet de Monvel's Algebra 31

4.1 Interior Operators . . . . . . . . . . . . . . . . . . . 32

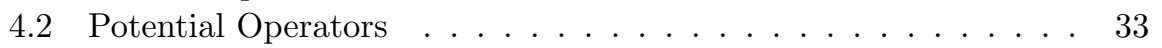

4.3 The Operators on the Boundary . . . . . . . . . . . . 34

4.4 Boutet de Monvel's Algebra . . . . . . . . . . . . . . . . . 36

4.5 Calderón Projectors . . . . . . . . . . . . . . 38

$5 \quad$ Basic Results 39

5.1 Pseudodifferential Boundary Problems . . . . . . . . . . . . . 39

5.2 The Free Resolvent . . . . . . . . . . . . . . . . . . . . . . . . 40

5.3 The Resolvent for the Problem with Boundary . . . . . . . . . . 42

5.4 Solution Operator . . . . . . . . . . . . . . . . . . 44

5.5 Scattered Plane Waves and Wave Operators . . . . . . . . . . . . 45

5.6 The S-Matrix and Scattering Poles . . . . . . . . . . . . . 46

6 Mapping Properties $\quad 49$

6.1 The Layer Potentials . . . . . . . . . . . . . . . . . . . . . . . . . 49

6.2 The Single Layer Potential . . . . . . . . . . . . . . . . . . . . . . . 54

6.3 The Double Layer Potential . . . . . . . . . . . . . . . . 59 
7 The Parametrix $\quad 65$

7.1 Ellipticity and Parametrix Construction . . . . . . . . . 65

7.2 Example: Square as a Scattering Object . . . . . . . . . . . 67

7.3 Proof of the Inequality . . . . . . . . . . . . . . . . . 69

8 Outlook: Three Dimensions $\quad 71$

Appendix $\quad \mathbf{7 2}$

A.1 Singularities in Boundary Value Problems . . . . . . . . . . 75

A.1.1 Green's Identity _. . . . . . . . . . . . . . . . 75

A.1.2 Regularity of Solutions of Cone-Degenerate BVPs . . . 76

A.2 Jensen's Formula . . . . . . . . . . . . . . . . . . . 77

A.3 Analytic Fredholm Theorem . . . . . . . . . . . . . . . . 78

A.4 Trace Class Operators . . . . . . . . . . . . . . . . 78

A.5 Carl's Inequality . . . . . . . . . . . . . . . . . . . 79

A.6 A Technical Lemma . . . . . . . . . . . . . . . . 79

$\begin{array}{ll}\text { Bibliography } & 82\end{array}$ 


\section{Chapter 1}

\section{Introduction}

This thesis deals with the problem of obstacle scattering theory in two spatial dimensions. ${ }^{1}$ The goal is to derive an upper bound for the counting function of the scattering poles in the case of a single, polygonal obstacle. The counting function counts the number of scattering poles on the meromorphic continuation of the scattering matrix which, in even dimensions, lives on the logarithmic cover of $\mathbb{C} \backslash\{0\}$.

Although investigators have always implicitly made this distinction, we would like to mention explicitly that one has to carefully distinguish between to types of poles in the context of scattering theory. Namely:

- resolvent resonances, and

- scattering resolvances,

the former being the poles of the cut-off resolvent, the latter of the meromorphic continuation of the scattering matrix.

The investigation of upper bounds for the counting function has started with R. Melrose's seminal work [Mel83] on the Schrödinger equation. Since then, researchers have extended this area of research by investigating various types of potentials, improving the bounds, or by including the search for lower bounds into their work. Some publications in these directions are [Zwo89], [Bar99], [Chr02], [CH05], and [Chr06]. Bounds for scattering in an even number of spatial dimensions have been investigated in [Int86]. For an overview up to the year 1994, consider the survey [Zwo94]. The educational paper [His12] also gives a comprehensive overview. Other lines of investigations included considering perturbations of the underlying metric, e.g., [Vod91b], [Vod91a], and [Vod92].

The topic of object scattering, however, seems to have been rather neglected: Except for [Mel84], progress has been scarce.

Only very recently, however, there has been a renewed interest in this theme ([CH10], [CH14b], [CH14a], and [Chr15]). T. Christiansen and P. D. Hislop have published various estimates for the counting function, based on the idea of black box scattering. Their method avoids the difficult technicalities of layer potentials by considering a self-adjoint perturbation $P$ which is defined outside

\footnotetext{
${ }^{1}$ We shall note that, when occasionally using " $n$ dimensions", we actually mean $n$ space dimensions.
} 
a connected open set $U \subset \mathbb{R}^{n}$ as

$$
\left.P u\right|_{\mathbb{R}^{n} \backslash U}=-\left.\Delta\right|_{\mathbb{R}^{n} \backslash U}
$$

for all $u \in \mathcal{D}(P)$. Using this abstract framework, they were, e.g., able to determine the maximal order of growth of the counting function in even dimensions. The general problem of scattering for non-smooth obstacles has been investigated by C. H. Wilcox [Wil75] for Neumann-type homogeneous boundary conditions, and by P. D. Lax and R. S. Phillips in [LP78], extending their infamous theory of scattering to domains which satisfy the so-called compactness property.

Having given a short overview of the history of the subject, we can state the two main ingredients which we will need in order obtain an upper bound. Those are:

1. The formulation of scattering theory in two dimensions by P. D. Lax and R. D. Phillips [LP72].

2. The introduction of Mellin pseudodifferential calculus [Sch98].

The theory of scattering by P. D. Lax and R. D. Phillips, also known as LaxPhillips theory, is well-known among mathematical analysts. Introduced in [LP64] and widely disseminated by their monograph [LP67], it is applicable to the wave equation, but also to symmetric hyperbolic systems as well as the Schrödinger equation [LP67]. Lesser known, however, is their extension to systems in even dimensions [LP72] and to non-smooth domains [LP78]. The latter two papers provide the basis for our investigation, as the techniques used in them allow us to analytically continue the S-matrix to the Riemann surface of the logarithm.

The elliptic theory for pseudodifferential operators for singular domains is delicate and quite demanding (cf. [RS89],[Sch91],[EB97], and [Sch98]). Elliptical pseudodifferential theory in singular domains has been developed by different groups of researchers, among the main proponents the school around Schulze and Rempel which has been heavily influenced by Russian scholars, the other being the $b$-calculus, developed by American researchers around Melrose. We have chosen Schulze's calculus (sometimes also called the Mellin calculus) for its usage of the Mellin transformation. Although the investigation of singularities for elliptic problems does not necessarily require methods of Mellin pseudodifferential calculus (cf. [Dau88], [Gri85], and [Gri92]), in our case it is necessary for the construction of a parametrix for the Calderón projector $\mathrm{C}_{01}(\lambda)$, an integral operator which lives on the boundary of the obstacle. Furthermore, we will need pseudodifferential methods for the reformulation of the single and double layer potential operators as potential operators in the sense of Schrohe and Schulze (cf. [SS94] and [SS95]).

This leads us to the second pillar of this thesis which involves using the ideas of Boutet de Monvel's calculus in conical domains. The first advantage, is the conceptual simplicity which emerges by just rewriting the scattering problem in terms of Boutet de Monvel's algebra. The solution operators, i.e. the residual operator of the Laplacian in the exterior domain $\Omega$ and the layer potentials are identified with the interior and potential operators of the algebra, respectively. As for the radiation condition, it can be formulated as an operator and regarded 
as a part of the algebra's trace operator. As it happens quite often in the course of science, this idea turned out quite fruitful since E. Schrohe and B.-W. Schulze have developed a theory of Boutet de Monvel algebras for spaces with conical singularities ([SS94] and [SS95]). Their toolbox turned out as useful by providing both accurate definitions and propositions about the continuity of potential operators.

In addition to the mentioned methods, we also make use of some results pioneered by the mathematical physics community, which involve identifying $\mathbb{R}^{2}$ with $\mathbb{C}$ and parametrizing the vicinity of the vertices with complex radial coordinates. Petri Ola and Lassi Päivärinta considered one dimensional graphs and applied the methods of Mellin calculus to the single layer potential of the standard Laplacian in [OP04]. This resulted in the explicit calculation of the Mellin symbol of the Laplacian's single layer potential, thereby revealing its pole structure.

\section{The Formulation of the Problem}

Consider the scattering of acoustic waves by a single obstacle $\mathcal{O}$ with boundary $\partial \Omega$. Denote the exterior domain by $\Omega$. The boundary shall be polygonal with edges of class $C^{\infty}$. Then, assuming the outgoing Sommerfeld radiation condition, the problem may be posed as

$$
\left\{\begin{aligned}
\left(\Delta+\lambda^{2}\right) u & =0 \\
\left.u\right|_{\partial \Omega} & =g, \\
\lim _{r \rightarrow \infty} r^{\frac{n-1}{2}}\left(\partial_{r} u-i \lambda u\right) & =0 .
\end{aligned}\right.
$$

In two dimensions, the scattering matrix $\hat{\mathrm{S}}(\sigma)$ admits an meromorphic continuation to the Riemann surface of the logarithm, $\Lambda$. The poles of this meromorphic continuation are called scattering resonances. Let $\Lambda_{m} \subset \Lambda$ be the $m^{\text {th }}$ sheet of $\Lambda$. We may identify this sheet with the upper or lower half of the complex plane, i.e. $\Lambda_{m}^{ \pm} \cong \mathbb{C}_{ \pm}$.

Theorem (Main theorem). The counting function $N_{m}^{ \pm}(r)$, which counts the scattering poles within a radius $r>0$ of the origin on the $m^{\text {th }}$ sheet, satisfies the inequality

$$
N_{m}^{ \pm}(r) \leq C r^{2}
$$

We will require the edges at the corners to be straight. This simplifies the proofs considerably, as they are technical even in this simplified situation.

\section{Outline of the Proof}

The basic idea of the proof is to find an analytic function that has zeros at exactly the same locations and of the same multiplicity as the scattering resonances. Using this analytic function, we can apply Jensen's formula and derive an upper estimate.

Let $r>0, h(z)$ be a meromorphic or holomorphic function, and $\left\{z_{k}\right\}_{k \in \mathbb{N}}$ a countable family of roots for $h(z)$. Then Jensen's formula states that 


$$
N_{h}(r) \leq \frac{1}{2 \pi \log (2)} \int_{0}^{2 \pi} \log \frac{\left|h\left(2 r e^{i \theta}\right)\right|}{|h(0)|} d \theta .
$$

In the case of scattering with Dirichlet boundary conditions, a function which suits our needs is the functional determinant

$$
d(\lambda)=\operatorname{det}(\mathbb{1}+\mathscr{R}(\lambda)),
$$

where $\mathscr{R}(\lambda)$ is the remainder of the parametrix $\mathrm{Q}$ applied to $\mathrm{C}_{01}(\lambda)$, the boundary limit of the single layer potential:

$$
\mathrm{Q}_{01}(\lambda)=\mathbb{1}+\mathscr{R}(\lambda)
$$

R. B. Melrose has proved the following identity for the case of an obstacle with smooth boundary using the well-known methods of Fourier pseudodifferential operators:

Proposition ([Mel84]). Consider the above scattering problem (1.1) for $n=3$ and an obstacle with smooth boundary $\partial \Omega$. If $\Delta_{\partial \Omega}$ is the induced Laplacian on $\partial \Omega$, then

$$
\left(1+\Delta_{\partial \Omega}\right)^{\frac{1}{2}} \mathrm{C}_{01}(\lambda)=1+\mathscr{R}(\lambda)
$$

where $\mathscr{R}(\lambda)$ is an entire family of pseudodifferential operators of order -1 such that -1 is an eigenvalue of $\mathscr{R}\left(\lambda_{j}\right)$ with algebraic multiplicity at least that of $\lambda_{j}$ as a pole of $\mathscr{R}(\lambda)$.

Consider the functional determinant

$$
d(\lambda)=\operatorname{det}\left(\mathbb{1}+\mathscr{R}^{n}(\lambda)\right) \text {, with } n=\operatorname{dim}(\partial \Omega)+1 .
$$

It is $d\left(\lambda_{j}\right)=0$ with multiplicity for the scattering poles $\lambda_{j}$. Using Jensen's formula (theorem A.2.1) for the function $d(\lambda)$ yields the upper bound

$$
|d(\lambda)| \leq C e^{C|\lambda|^{n}}
$$

In order to generalize this result to polyhedral obstacles, we have to introduce the machinery of Singular Analysis. This means that the central role of pseudodifferential operators based on the Fourier transformation will be substituted with Mellin pseudodifferential operators. The latter are based on the Mellin transformation, i.e. a transformation of the form

$$
\mathcal{M} u(z)=\int_{0}^{\infty} r^{z-1} u(r) d r, \quad z \in \mathbb{C}, u \in C_{0}^{\infty}\left(\mathbb{R}_{+}\right) .
$$

The drawback of applying Mellin pseudodifferential calculus is its higher complexity, even in the simple case of a one dimensional boundary $\partial \Omega$. Choosing the Mellin calculus as our main tool yields two problems:

(i) Finding appropriate function spaces for both the boundary and the interior and exterior domains.

(ii) Defining an operator algebra in which we can embed the potential operators and their interior and exterior limits on the boundary. 
Fortunately, we are able to make use of B.-W. Schulze and E. Schrohe's research on Boutet de Monvel algebras in [SS94] and [SS95]. Their development of a symbolic calculus for boundary value problems in conical domains provides a fertile environment for the study of layer potentials. Although we do not need the full calculus of Boutet de Monvel-algebras, their formulation of Boutet de Monvel-valued symbols provides a useful line of thought. We attempt to reformulate the single and double layer potentials as potential operators in the sense of Schulze and Schrohe and study their interior and exterior limits on the polygonal boundary $\partial \Omega$. These limits turn out to be Mellin pseudodifferential operators which operate on the boundary and can be considered as the lower right entries of the elements of Boutet de Monvel algebra.

In general, all the operators we use in the course of our work, let them be potential or boundary operators, are of the form

$$
\mathrm{A}=\mathrm{A}_{\mathrm{M}}+\mathrm{A}_{\psi}+\mathrm{A}_{\mathrm{G}} .
$$

Here, the term $\mathrm{A}_{\psi}$ is certainly most familiar to the reader. It is a standard (i.e. Fourier) pseudodifferential operator defined on the interior of the edges, where the curved parts are bounded away from the corners.

In contrast to this, the terms $\mathrm{A}_{\mathrm{M}}$ denotes the Mellin contribution of the operator in question. It is basically an operator of the form

$$
\mathrm{op}_{\mathrm{M}}^{\gamma}[h] u=\mathcal{M}_{\gamma}^{-1} h(r, z) \mathcal{M}_{\gamma} u, \quad u \in C_{0}^{\infty}\left(\mathbb{R}_{+}\right)
$$

where $h(r, z)$, the Mellin symbol, is a function which is holomorphic or meromorphic in the variable $z$ (with additional properties). In general, this so-called Mellin symbol is also dependent on several more variables, such as parametrizations of the edge. But since we require the edges in a neighborhood of the corners to be straight, the Mellin symbols will mostly depend on solely one variable $\vartheta$ which parametrizes an angle.

Finally, the term $\mathrm{A}_{\mathrm{G}}$ is the so-called Green operator. It is smoothing and has been included into the calculus of Mellin pseudodifferential operators for composition purposes and is named by analogy after the Green operator which is part of Boutet de Monvel's algebra for boundary value problems.

One of the drawbacks for Mellin pseudodifferential and potential operators in our situation is that the Mellin symbols are not scalar valued, but rather matrixvalued. To be more precise, a Mellin symbol for a potential operator in a wedgelike domain $X^{\wedge}$ has the form $\bar{h}=\left(h_{1}, h_{2}\right)$, a $1 \times 2$-matrix, which reflects the fact that the boundary of the domain without the origin consists of two semi-lines: $\partial X^{\wedge}=Y_{1} \uplus Y_{2}$.

The Mellin symbols of the operator on the boundary, on the other hand, are given by the $2 \times 2$-matrices

$$
\hat{h}=\left[\begin{array}{ll}
h_{11} & h_{12} \\
h_{21} & h_{22}
\end{array}\right],
$$

where the off-diagonal entries are so-called smoothing Mellin symbols. These peculiarities require some effort in notation and book-keeping. As we have already suggested, matrices will be denoted, depending on their type, by a 'bar' or a 'hat'. This also affects the frequently used cut-off functions which will often appear as diagonal matrices $\hat{\phi}$. All these aspects will be explained in greater detail in chapter 4 . 
The algebra of Boutet de Monvel does in fact play a small role in chapter 4 of this thesis. Although certainly a triviality, we attempt to reformulate the scattering problem (1.1) and its solution operator in terms of Boutet de Monvel, thereby introducing the notions of the potential and interior operators, the former being sometimes also called Poisson operators. It seems to make the concept of the solution for scattering problems as done in the treatise [Tay13] conceptually simpler, at least for the uninitialized reader.

The fundamental solution plays a central role since it and its normal derivative are the operator kernels of the single and double layer potentials. The study of these kernels is contained in chapter 6 . Although a boundary of dimensionality one does simplify the elliptic theory, in even dimensions it is essentially given by the Hankel functions of first and second kind, and thereby add again more complexity due to their more involved expansions. For example, for two dimensions, it is given by (denoting the outgoing solution with the superscript 'o' and the incoming solution by the superscript ' $i$ '):

$$
r_{0}^{*}(\lambda|x-y|)= \begin{cases}-\frac{i}{4} H_{0}^{(2)}(\lambda|x-y|), & \text { for } *=\mathrm{o}, \\ +\frac{i}{4} H_{0}^{(1)}(\lambda|x-y|), & \text { for } *=\mathrm{i} .\end{cases}
$$

for $x \neq y \in \mathbb{R}^{2}$ and $\lambda \in \Lambda$. Then, in chapter 6 , we will demonstrate that the layer potentials are indeed elements of the class of potential operators on $\Omega$, $\mathcal{P}^{\mu}(\Omega ; \Lambda), \mu \in \mathbb{R}$. Furthermore, we will show that there exists an expansion of the form

$$
\mathrm{Sl}^{\mathrm{i} / \mathrm{o}}(\lambda)-\sum_{k=0}^{M} \frac{(-1)^{k}}{(k !)^{2}}\left(\frac{\lambda}{2}\right)^{2 k} \mathrm{~T}_{\log , k} \in \mathcal{P}^{M+1}(\Omega ; \Lambda)
$$

and

$$
\mathrm{Dl}^{\mathrm{i} / \mathrm{o}}-\tilde{\mathrm{T}}_{\text {sing }}-\sum_{k=0}^{M} \frac{(-1)^{k}}{(k !)^{2}}\left(\frac{\lambda}{2}\right)^{2 k} \tilde{\mathrm{T}}_{\log , k} \in \mathcal{P}^{M+1}(\Omega ; \Lambda),
$$

where $\mathrm{T}_{\log , k}, \mathrm{~T}_{\text {sing }}$, and $\tilde{\mathrm{T}}_{\log , \mathrm{k}}, k \in \mathbb{N}_{0}$ are certain integral operators. The details of this will be explained in the four Theorems 6.7 to 6.13 .

In the penultimate step, we have to apply the parametrix to the above families of operators. It turns out, due to a factorization of the Mellin symbols, that these operators map classical fractional Sobolev spaces $H^{s}(\partial \Omega)$ onto Sobolev spaces of higher regularity, e.g.,

$$
\mathrm{QT}_{\log , k}: H^{s}(\partial \Omega) \rightarrow H^{s+2 k}(\partial \Omega) .
$$

The subsequent approximations are based on Weyl's convexity estimate (Theorem A.4.3):

$$
|d(\lambda)| \leq \prod_{j=1}^{\infty}\left(1+\chi_{j}(\mathscr{R}(\lambda))\right)
$$

The singular values $\chi_{j}(\mathscr{R}(\lambda))$ themselves are approximated by exploiting the above expansion of the boundary operators and then approximating the numbers $\chi_{j}\left(\mathrm{QT}_{k}\right)$ again by their operator norm and the entropy numbers of the embeddings $H^{2 k}(\partial \Omega) \rightarrow L^{2}(\partial \Omega)$. This is the content of Carl's Inequality (Theorem A.5.1). The concept of entropy numbers is explained in the appendix in section A.5. 


\section{Outline}

- Chapter 2 will introduce the reader to the general concepts of scattering theory. While the first section mentions the most general algebraic aspects, such as wave operators, the notions in subsection 2.2 are more specific to the theory of obstacle scattering. Although some of them will be reintroduced in chapter 5, we aimed for preparing the reader's mind and chose redundancy over brevity.

- Chapter 3 will introduce the necessary function spaces and some theory of Mellin pseudodifferential operators on the positive real axis. The results form the technical basis for the algebra of boundary operators in the subsequent chapter.

- Chapter 4 will provide the reader with the definitions of the operator classes. We will focus on the potential and boundary operators since these are the classes which the layer potentials belong to. In addition, we outline the algebra of Boutet de Monvel for boundary value problems for conical singularities without going into details. Some understanding of standard pseudodifferential operators will be required.

- In chapter 5 we establish basic results about scattering theory for polygonally bounded obstacles. By modifying an argument by Lax and Phillips [LP72], we provide a proof for the existence of the resolvent operator for the exterior problem for polygonal boundaries. The results on the wave operators and the S-matrix are standard results, which only need a trivial modification by incorporating Green's identities for polyhedral domains.

- Chapter 6 is thematically divided into two parts: The first one deals with the asymptotic expansions of the fundamental solutions. The fundamental solution and its normal derivative are the kernels of the single and double layer potentials. Investigating their expansions for $|x-y| \rightarrow 0$ and $|x| \rightarrow \infty$ provides a basis for the analysis of layer potentials in the subsequent part of the chapter. There, we will show that the layer potentials are potential operators in the sense of chapter 4 . We will understand their mapping properties and their exterior and interior limits on the boundary. An important result is the derivation of modified jump relations for the double layer potential.

- Chapter 7 focuses on the proof of the main theorem. We will construct a parametrix for the boundary limit of the single layer potential. We provide the reader with a simple example: The explicit construction of the parametrix for the boundary of a square in two dimensions. The remainder of this chapter is dedicated to the proof of the upper estimate. This chapter is very technical and requires some knowledge about entropy numbers. Their basic properties are provided in the appendix.

- Finally, chapter 8 will give the reader an outlook onto the problem in three dimensions. It turns out that the elliptic theory is much more involved, whereas the scattering problem itself simplifies considerably. 


\section{Chapter 2}

\section{Fundamentals of Scattering Theory}

In this chapter we will outline the fundamental ideas of scattering theory. In section 2.1 we introduce the most basic principles of scattering theory, i.e. the idea of comparing a free and a perturbed problem with each other. Section 2.2 is a short account on the standard notions of obstacle scattering theory.

\subsection{Abstract Scattering Theory}

Consider the pair $(\mathrm{H}, \mathcal{H})$ which consists of a Hilbert space $\mathcal{H}$ and a self-adjoint operator $\mathrm{H}: \mathcal{D}(\mathrm{H}) \rightarrow \mathcal{H}$, with $\mathcal{D}(\mathrm{H}) \subseteq \mathcal{H}$ being the domain of the operator $\mathrm{H}$. According standard spectral theory, the Hilbert space $\mathcal{H}$ may be decomposed as a direct sum of the three subspaces:

$$
\mathcal{H} \cong \mathcal{H}^{p p} \oplus \mathcal{H}^{s c} \oplus \mathcal{H}^{a c} .
$$

Here, $\mathcal{H}^{p p}$ denotes the pure point spectral eigenspace of $\mathrm{H}, \mathcal{H}^{s c}$ the singular continuous eigenspace, and $\mathcal{H}^{a c}$ the absolutely continuous eigenspace.

The absence of the singular continuous eigenspace is a feature of realistic physical systems. Up to this point, no realistic systems are known where the singular continuous spectral eigenspace is non-trivial. The states which are contained in the singular continuous spectrum and the absolutely continuous eigenspaces can be given physical interpretations by means of the so-called RAGE theorem. The objective of scattering theory is to understand the absolutely continuous spectrum of the full Hamiltonian $\mathrm{H}$. In order to do this, one considers a pair $\left(\mathrm{H}_{0}, \mathcal{H}_{0}\right)$ of a free Hilbert space and free Hamiltonian $\mathcal{H}_{0}$, the latter again being a self-adjoint operator. One seeks to construct a unitary equivalence between the absolutely continuous eigenspace $\mathcal{H}^{a c}$ and the free Hilbert space $\mathcal{H}_{0}$. Here, we are already implying that $\mathcal{H}_{0}=\mathcal{H}_{0}^{a c}$, that is the free Hilbert space consists solely of the absolutely continuous subspace of $\mathrm{H}_{0}$.

Since both $\mathrm{H}$ and $\mathrm{H}_{0}$ are self-adjoint operators, they give rise to one-parameter strongly continuous unitary groups $\left\{\mathrm{U}(t)=e^{-i \mathrm{H} t}\right\}_{t \in \mathbb{R}_{+}}$and $\left\{\mathrm{U}_{0}(t)=e^{-i \mathrm{H}_{0}}\right\}_{t \in \mathbb{R}_{+}}$, respectively. These groups are used to construct the wave operators which function as the unitary mappings between $\mathcal{H}$ and $\mathcal{H}_{0}$. 
In full generality, the wave operators are defined as

$$
\mathrm{W}_{\text {out }}\left(\mathrm{H}, \mathrm{H}_{0} ; \mathrm{J}_{0}, \mathrm{P}_{M}\right):=\underset{t \rightarrow \infty}{\mathrm{s}-\lim } e^{i \mathrm{H} t} \mathrm{~J}_{0} e^{-i \mathrm{H}_{0} t} \mathrm{P}_{M},
$$

and

$$
\mathrm{W}_{\mathrm{in}}\left(\mathrm{H}, \mathrm{H}_{0} ; \mathrm{J}_{0}, \mathrm{P}_{M}\right):=\underset{t \rightarrow-\infty}{\mathrm{s}-\lim } e^{i \mathrm{H} t} \mathrm{~J} e^{-i \mathrm{H}_{0} t} \mathrm{P}_{M}
$$

if the limits exists. Here, $\mathrm{P}_{M}: \mathcal{H}_{0} \rightarrow \mathcal{H}_{0}$ is the projection onto a closed subspace $M \subseteq \mathcal{H}_{0}$, and the operator $\mathrm{J}_{0}: \mathcal{H}_{0} \rightarrow \mathcal{H}$ is an embedding operator. ${ }^{1}$

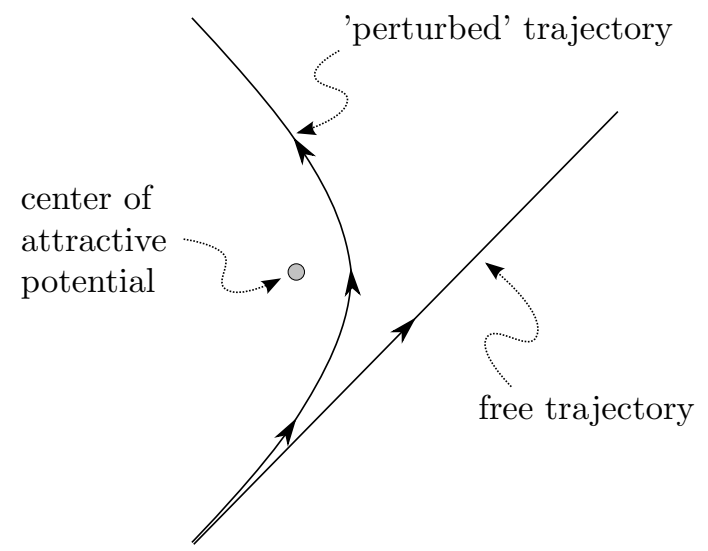

Figure 2.1: Free and perturbed trajectories of a classical particle

The wave operators relate the free solution, i.e. the solution of the 'easier' problem, to the perturbed problem. This is expressed by the intertwining property

$$
e^{i \mathrm{H} t} \mathrm{~W}_{\mathrm{i} / \mathrm{o}}\left(\mathrm{H}, \mathrm{H}_{0} ; \mathrm{J}_{0}, \mathrm{P}_{M}\right)=\mathrm{W}_{\mathrm{i} / \mathrm{o}}\left(\mathrm{H}, \mathrm{H}_{0} ; \mathrm{J}_{0}, \mathrm{P}_{M}\right) e^{i \mathrm{H}_{0} t} .
$$

This easier problem may, depending from case to case, be either a linearization of a non-linear problem, a Schrödinger equation without potential, or a trivial geometry.

Definition 2.1 (Completeness of Wave Operators). The wave operator $W_{\mathrm{i} / \mathrm{o}}\left(\mathrm{H}, \mathrm{H}_{0}\right)$ is called complete, if

$$
\operatorname{ran} \mathrm{W}_{\text {out }}\left(\mathrm{H}, \mathrm{H}_{0}\right)=\mathcal{H}^{\mathrm{ac}},
$$

asymptotically complete, if

$$
\mathcal{H}_{p p} \oplus \operatorname{ran} W_{\mathrm{i} / \mathrm{o}}\left(\mathrm{H}, \mathrm{H}_{0}\right)
$$

and weakly asymptotically complete, if

$$
\operatorname{ran} \mathrm{W}_{\text {out }}\left(\mathrm{H}, \mathrm{H}_{0}\right)=\operatorname{ran} \mathrm{W}_{\text {in }}\left(\mathrm{H}, \mathrm{H}_{0}\right) \text {. }
$$

\footnotetext{
${ }^{1} \mathrm{~A}$ variant of the identification operators is especially important for multichannel scattering theory.
} 
There are numerous techniques developed to deal with the problem of existence and completeness of wave operators. Most notable is Cook's lemma which is the foundation of almost all theorems on the existence of these operators. Furthermore, there are trace class methods by Kato, Rosenblum, and Pearson. And finally, the techniques by Enss with further simplifications by Davies and Perry. The scattering operator which relates the solutions of the incoming free space to solutions in the outgoing free space is defined by

$$
\mathrm{S}:=\mathrm{W}_{\text {out }}\left(\mathrm{H}, \mathrm{H}_{0}\right)^{*} \mathrm{~W}_{\text {in }}\left(\mathrm{H}, \mathrm{H}_{0}\right) .
$$

The scattering operator $\mathrm{S}$ is decomposable in $L^{2}\left(\mathbb{R}_{+}, \sigma^{n-1} d \sigma ; L^{2}\left(\mathbb{S}^{n-1}\right)\right)$, i.e. $\{\mathrm{S}(k)\}_{k \in \mathbb{R}}$ being the direct integral of the operator S. The operator for fixed $\sigma \in \mathbb{R}$ is being called the scattering matrix. It is the S-matrix which may be meromorphically continued to either the complex plane (odd dimensions) or the Riemann surface of the logarithm (even dimensions).

\subsection{Obstacle Scattering}

\subsubsection{Formulation of the Problem}

Let $\mathcal{O} \subset \mathbb{R}^{2}$ be a compact obstacle, its complement $\Omega^{\mathrm{c}}=\mathbb{R}^{2} \backslash \Omega$ connected. In the classical theory of obstacle scattering, one usually assumes that its boundary, $\partial \Omega$, is smooth. Hence, for $n$ spatial dimensions, the scattering problem for steady-states can be formulated by

$$
\left\{\begin{aligned}
\left(\Delta+\lambda^{2}\right) u & =0, \\
\left.u\right|_{\partial \Omega} & =f, \\
\lim _{r \rightarrow \infty} r^{\frac{n-1}{2}}\left(\partial_{r} u \mp i \lambda u\right) & =0 .
\end{aligned}\right.
$$

One has the choice between different boundary conditions, e.g., one has the Dirichlet condition, the Neumann boundary condition, and the so-called Robin condition, which is given by

$$
\partial_{\nu} u(x)=\alpha(x) \cdot u(x),
$$

$\alpha$ being a smooth function on the boundary, i.e. $\alpha \in C^{\infty}(\partial \Omega)$.

The last equation of (2.2) implies that there are two different possibilities: The minus sign designates the incoming Sommerfeld radiation condition, if there is a plus sign, one speaks of the incoming Sommerfeld radiation condition.

\subsubsection{Spectral Theory}

The limiting absorption principle allows one to construct families of generalized eigenfunctions and thereby construct spectral representations for the self-adjoint operators $\mathcal{H}$ and $\mathcal{H}_{0}$. In the case of the free Laplacian in $\mathbb{R}^{n}$, the spectral representation is the well-known Fourier transformation. That is,

$$
\begin{gathered}
\Phi: L^{2}\left(\mathbb{R}^{n}\right) \rightarrow L^{2}\left(\mathbb{R}^{n}\right), \\
\Phi f(k)=\frac{1}{(2 \pi)^{n / 2}} \int_{\mathbb{R}^{2}} e^{-i k x} f(x) d x,
\end{gathered}
$$


where the integral is to be understood as a $L^{2}$-limit. One can then easily see that

$$
\Phi(-\Delta) \Phi^{*}=|k|^{2}, \quad k \in \mathbb{R}^{3} .
$$

We will recast this representation in the language of scattering theory as follows: Denote the set of plane wave functions by

$$
\psi_{0}(x, \omega, \sigma)=\frac{1}{(2 \pi)^{n / 2}} e^{-i x \omega \sigma}, \quad x \in \mathbb{R}^{3},(\sigma, \omega) \in \mathbb{R}_{+} \times \mathbb{S}^{1}
$$

We make the observation that the plane waves satisfy both the reduced wave equation and the outgoing Sommerfeld condition:

$$
\left\{\begin{aligned}
\left(\Delta+\lambda^{2}\right) \psi_{0}(x, k) & =0, \\
\lim _{r \rightarrow \infty} r^{\frac{n-1}{2}}\left(\partial_{r} \psi_{0}-i \lambda \psi_{0}\right) & =0 .
\end{aligned}\right.
$$

The set $\left\{\psi_{0}(x, k) \mid x, k \in \mathbb{R}^{n}\right\}$ forms a family of generalized eigenfunctions for the free Laplacian $-\Delta_{\mathbb{R}^{n}}$ in $L^{2}\left(\mathbb{R}^{n}\right)$. The Fourier transformation can then using radial coordinates in Fourier space - be rewritten as

$$
\Phi f(k)=\int_{\mathbb{R}^{n}} \overline{\psi_{0}(x, \omega, \sigma)} f(x) d x .
$$

In order to construct similar spectral representation for the Laplacian on a domain with obstacle, one has to construct a family of generalized eigenfunctions and define a modified Fourier transformation. Assuming a Laplacian with Dirichlet boundary conditions, we solve the following equations by utilizing the Limiting Absorption principle:

$$
\left\{\begin{array}{rlrl}
\left(\Delta_{x}+\lambda^{2}\right) v^{\mathrm{i} / \mathrm{o}}(x, \omega, \sigma) & =0, & & x \in \Omega, \\
v^{\mathrm{i} / \mathrm{o}}(x, \omega, \sigma) & =-e^{i \lambda x \omega}, & x \in \partial \Omega, \\
\lim _{r \rightarrow \infty} r^{\frac{n-1}{2}}\left(\partial_{r} v \mp i \lambda v^{\mathrm{i} / \mathrm{o}}\right) & =0
\end{array}\right.
$$

These equations however, yield two distinct families of generalized eigenfunctions, each one fulfilling the outgoing and incoming radiation condition, respectively. Denote these families by $\left\{\psi^{\mathrm{i} / \mathrm{o}}(x, \omega, \sigma) \mid(x, \omega, \sigma) \in \Omega \times \mathbb{R}_{+} \times \mathbb{S}^{1}\right\}$, where 'i' stands for incoming and 'o' for outgoing. Generalized eigenfunctions of this kind are called scattered waves.

Adding the a plane wave to the scattered waves yields the distorted plane waves:

$$
\psi^{\mathrm{i} / \mathrm{o}}(x, \omega, \sigma)=e^{-i \sigma x \omega}+v^{\mathrm{i} / \mathrm{o}}(x, \omega, \sigma) .
$$

The families $\left\{\psi^{\mathrm{i} / \mathrm{o}}(x, \omega, \sigma) \mid(x, \omega, \sigma) \in \Omega \times \mathbb{R}_{+} \times \mathbb{S}^{n-1}\right\}$ can be used to to define spectral representations analogously to $(2.4)$, namely $\Phi_{\mathrm{i} / \mathrm{o}}: L^{2}(\Omega) \rightarrow L^{2}\left(\mathbb{R}^{n}\right)$ with

$$
\Phi_{\mathrm{i} / \mathrm{o}} f(\omega, \sigma):=\frac{1}{(2 \pi)^{n / 2}} \int_{\Omega} \overline{\psi^{\mathrm{i} / \mathrm{o}}(x, \omega, \sigma)} f(x) d x .
$$

The integral is again to be understood in the $L^{2}$-sense. In order to define the wave operators for the scattering problem, we may utilize the spectral representations $\Phi$ and $\Phi_{\mathrm{i} / \mathrm{o}}$ by setting

$$
\mathrm{W}_{\text {out }}:=\mathrm{W}_{\text {out }}\left(\mathrm{H}, \mathrm{H}_{0}\right):=\Phi \Phi_{\text {out }}^{*},
$$

and

$$
\mathrm{W}_{\mathrm{in}}:=\mathrm{W}_{\mathrm{in}}\left(\mathrm{H}, \mathrm{H}_{0}\right):=\Phi \Phi_{\mathrm{in}}^{*} \text {. }
$$




\subsubsection{The Scattering Matrix}

The scattering operator is then, following the abstract definition (2.1), given by

$$
\mathrm{S}=\mathrm{W}_{\text {out }}^{*} \mathrm{~W}_{\text {in }},
$$

and is therefore a bounded operator $B\left(L^{2}\left(\mathbb{R}^{n}\right)\right)$. Set $\hat{S}:=\Phi S \Phi^{*}$. Then one can that the scattering operator commutes with the spectral measure $\left\{\Pi_{0}(\sigma)\right\}_{\sigma \in \mathbb{R}_{+}}$ of the free Hamiltonion:

$$
\Pi_{0}(\sigma) \hat{\mathrm{S}}=\hat{\mathrm{S}} \Pi_{0}(\sigma), \quad \sigma>0
$$

This commutation relation has a interesting consequence (cf. [RS78] for a more detailed account). Namely, one can show that $\hat{\mathrm{S}}$ is decomposable:

Proposition 2.2. The Fourier transform of the scattering operator is decomposable, i.e. there is a family of operators $\hat{\mathrm{S}}(\sigma), \sigma \in \mathbb{R}_{+}$, such that

$$
(\varphi, \hat{\mathrm{S}} \psi)=\int_{\mathbb{R}_{+}}\langle\varphi(\sigma), \hat{\mathrm{S}}(\sigma) \psi(\sigma)\rangle_{L^{2}\left(\mathbb{S}^{n-1}\right)} d \sigma, \quad \varphi, \psi \in L^{2}\left(\mathbb{R}^{n}\right) .
$$

The operators $\hat{\mathrm{S}}(\sigma)$ are called the fibers of $\hat{\mathrm{S}}$ at $\sigma$. One can go further and show that the fibers (assuming two spatial dimensions) have the following form:

$$
\hat{\mathrm{S}}(\sigma)=\mathbb{1}+\left(\frac{\sigma}{2 \pi i}\right)^{\frac{1}{2}} \mathrm{~A}(\sigma) .
$$

This will be discussed in chapter 5 . It is a well known fact that for an odd number of spatial dimensions the S-matrix can be analytically continued to the complex plane $\mathbb{C}$. Specifically Lax-Phillips theory in odd dimensions provides powerful theorems which guarantee the existence and under quite general conditions (cf. [LP67] and [LP78] for modifications incorporating domains satisfying the finite tiling property). The counting function for the scattering poles can then simply be defined as the number of scattering poles within a ball $\mathbb{B}_{r}(0)$ of radius $r>0$ and center 0 in the complex plane $\mathbb{C}$.

For an even number of spatial dimensions however, the S-matrix can be analytically continued to a meromorphic function on the Riemann surface of the logarithm, $\Lambda$. In order to define the counting function on $\Lambda$, one needs to select a specific sheet first, $\Lambda_{m} \subset \Lambda$, first. Define

$$
\begin{gathered}
\Lambda_{m}^{+}:=\left\{r e^{i \phi} \mid r>0,2 \pi m<\phi<(2 m+1) \pi\right\} . \\
\Lambda_{m}^{-}:=\left\{r e^{i \phi} \mid r>0,(2 m-1) \pi<\phi<2 \pi\right\} .
\end{gathered}
$$

The definition of the counting function for an even number of spacial dimension is then as follows:

Definition 2.3. The resonance counting function for even spatial dimensions, $N_{m}^{ \pm}(r)$, for the $m^{\text {th }}$ sheet $\Lambda_{m}, m \in \mathbb{Z}$, is defined as the number of poles $\lambda_{j}$ of $\mathrm{R}^{\mathrm{i} / \mathrm{o}}(\lambda)$ with modulus less or equal than $r$, i.e. $\left|\lambda_{j}\right| \leq r$ and $m \pi<\arg \lambda_{j}<(m+1) \pi$. 


\section{Chapter 3}

\section{Fundamentals of Mellin Calculus}

Having introduced the necessary foundations of scattering theory, we now have to take a look at the elliptic theory. As we have already mentioned in the introduction, the elliptic theory for one dimensional graphs is considerably simpler than the higher dimensional ones. Still, even in the one dimensional case we have to invent a readable notation for bookkeeping purposes. The ultimate goal will be to rewrite the boundary values of the single and double layer potentials in terms of this algebra. This will be the goal of chapter 6 .

Summarized, this chapter introduces technical preliminaries such as:

- Definitions and nomenclature of the obstacle's geometry (section 3.1),

- Function spaces (section 3.2),

- The definition of the Mellin pseudodifferential calculi (sections 3.3 and $4.3)$.

\subsection{Curvilinear Polygon in Two Dimensions}

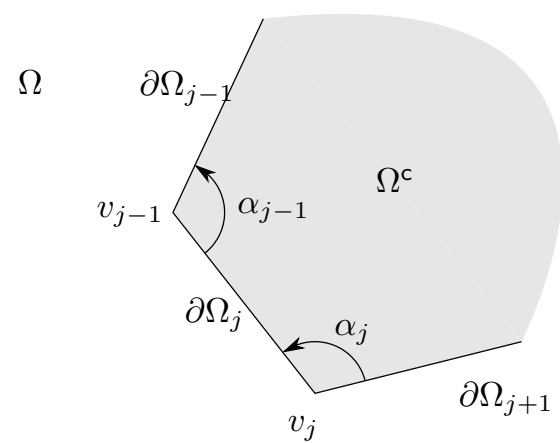

Figure 3.1: Naming convention of the edges and corners of the boundary 
We are dealing with two dimensional Euclidean scattering, i.e. the surrounding space is $\mathbb{R}^{2}$ with the standard Euclidean product $x \cdot y:=x_{1} y_{1}+x_{2} y_{2}, x, y \in \mathbb{R}^{2}$. The corresponding norm shall simply be defined by $|x|:=\sqrt{x \cdot x}, x \in \mathbb{R}^{2}$.

As we have mentioned in the introduction, we will denote the exterior domain by $\Omega$ and the obstacle by the complement $\Omega^{c}$. We consider the boundary of the obstacle to be piecewise smooth, i.e.

$$
\partial \Omega=\bigcup_{j=1}^{N} \overline{\partial \Omega_{j}},
$$

where each $\partial \Omega_{j} \subset M_{j}$ is a smooth hypersurface embedded in a larger hypersurface $M_{j}$. This definition is a special case of the Definition A.1.2 given by [Dau88]. Each edge $\partial \Omega_{j}$ may be parametrized by arc length, i.e. by a curve $x_{j}:\left(0, l_{j}\right) \rightarrow \partial \Omega_{j}, t \mapsto x_{j}(t)$, where $l_{j}>0$. Furthermore, this curve may be extended to $\left(-\epsilon, l_{j}+\epsilon\right) \mapsto M_{j}$. The vertices shall be denoted by $v_{j}, 1, \ldots, N$. The terms 'vertex' and 'corner' will be used synonymously.

Remark 3.1. It is very important to note that we assume the edges to be straight near the vertices. That is, for each vertex $v_{j}$, there is a neighborhood containing $v_{j}$ such that in this neighborhood, the edges $\partial \Omega_{j}$ and $\partial \Omega_{j+1}$ are straight semi-lines. This will make the subsequent calculations of the Mellin symbols easier.

We define the outer and inner limits for $u \in C^{\infty}\left(\mathbb{R}^{2}\right)$ and $t \in \partial \Omega$ by

$$
\begin{aligned}
\gamma_{0}^{+} u(t) & :=\lim _{\substack{x \rightarrow t \\
x \in \Omega}} u(x), \\
\gamma_{0}^{-} u(t) & :=\lim _{\substack{x \rightarrow t \\
x \in \Omega^{c}}} u(x) .
\end{aligned}
$$

We need to define normal vector fields for a piecewise smooth boundaries. The (outward pointing) normal vector field is only defined on the interior of the edges, i.e. piecewise:

$$
\partial_{\nu_{j}(t)}:=\nu_{j}(t) \cdot \nabla_{x}, \quad \text { for } t \in \partial \Omega_{j}, j=1, \ldots, N .
$$

The inward pointing normal vector field is then simply defined by $\nu_{j, x}^{-}:=-\nu_{j, x}^{+}$. Denote by $v \mapsto \partial_{v}$ the canonical isomorphism $C^{\infty}\left(\mathbb{R}^{2} ; \mathbb{R}^{2}\right) \cong \operatorname{Diff}^{1}\left(\mathbb{R}^{2}\right)$. Then $y \mapsto \tilde{\nu}_{y} \in \mathbb{R}^{2}$ is the normal vector field written as a field with values in $\mathbb{R}^{2}$ instead of a differential operator of order 1 via the canonical isomorphism.

$$
\gamma_{1}^{ \pm} u(t):=\gamma_{0}^{ \pm} \partial_{\nu} u(t)
$$

Notice that, in the case of piecewise smooth boundaries, we have to define the operator for each smooth segment separately, i.e.

$$
\gamma_{1}^{ \pm} u(t):=\sum_{j=1}^{N} \gamma_{0, \partial \Omega_{j}}^{ \pm} \partial_{\nu_{j}} u(t) .
$$

Of course, this shouldn't irritate us, and for this reason we will stick to notation (3.4). The boundary operator is then defined as the column vector

$$
\gamma^{ \pm}:=\left[\begin{array}{l}
\gamma_{0}^{ \pm} \\
\gamma_{1}^{ \pm}
\end{array}\right]
$$


We will be constantly using cut-off functions of the following sort: Consider a vertex $v_{j}, j=1, \ldots, N$. $\phi_{j}$ shall denote a function $C^{\infty}\left(\partial \Omega_{j}\right)$ which is identically one near the vertex $j$ and zero outside a small neighborhood. Setting $\theta_{j}:=$ $1_{\partial \Omega_{j}}-\phi_{j}^{j}-\phi_{j}^{j+1}$, we obtain a function $\theta_{j} \in C_{c}^{\infty}\left(\partial \Omega_{j}\right)$. The interior angles of the corners will be denoted by $\alpha_{1}, \ldots, \alpha_{N}$. Hence, we have a partition of unity of the graph $\partial \Omega$ : $\sum_{j}\left(\phi_{j}^{j+1}+\phi_{j}^{j}+\theta_{j}\right)=1_{\partial \Omega}$.

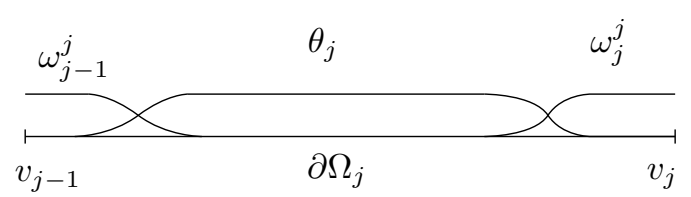

Figure 3.2: The cut-off functions on an interval

The parametrizations $x_{j}(\sigma)$ induce a metric on each edge of the boundary.

$$
D x_{j}(\sigma): \mathbb{R} \cong T_{\sigma} \mathbb{R} \rightarrow T_{p} \partial \Omega_{j} .
$$

Of course, in our simple case, $D x_{j}(\sigma)=x_{j}^{\prime}(\sigma) \in \mathbb{R}^{2}$ pointwise. We have

$$
\left|x_{j}^{\prime}(\sigma)\right|=\sqrt{x_{j}^{\prime}(\sigma) \cdot x_{j}^{\prime}(\sigma)} .
$$

This notation comes in handy when defining the Lebesgue space on the boundary below. We denote this norm as

$$
|\cdot|_{t}: T_{t} \partial \Omega_{j} \rightarrow[0, \infty)
$$

This notation will come in handy when we are dealing with pseudodifferential operators operators defined on the boundary. It is almost unnecessary to say that $T_{t} \partial \Omega_{j} \cong \mathbb{R}$.

This metric immediately defines a Lebesgue measure on the boundary. For $f: \partial \Omega_{j} \rightarrow \mathbb{C}$ measurable and a parametrization $x_{j}:\left[0, l_{j}\right] \rightarrow \partial \Omega_{j}$, we set

$$
\int_{\partial \Omega_{j}} f d s_{j}:=\int_{0}^{l_{j}}\left(f \circ x_{j}\right)\left|x_{j}^{\prime}(\sigma)\right| d \sigma .
$$

$L^{1}\left(\partial \Omega, d s_{j}\right)$ is then defined as the space of integrable functions. On the boundary $\partial \Omega_{j}$, we define the spaces $L^{2}\left(\partial \Omega_{j}, d s_{j}\right)$ with scalar product

$$
\left\langle f_{j}, g_{j}\right\rangle_{\partial \Omega_{j}}:=\int_{\partial \Omega_{j}} \overline{f_{j}} g_{j} d s_{j} .
$$

Finally, we may define the space $L^{2}(\partial \Omega, d s):=\bigoplus_{j=1}^{N} L^{2}\left(\partial \Omega_{j}, d s_{j}\right)$. The scalar product is given by

$$
\langle f, g\rangle:=\sum_{j=1}^{N}\left\langle f_{j}, g_{j}\right\rangle_{\partial \Omega_{j}} .
$$

Notice, that we have avoided the subscript $\partial \Omega$ for the $L^{2}$-product on the whole boundary. 


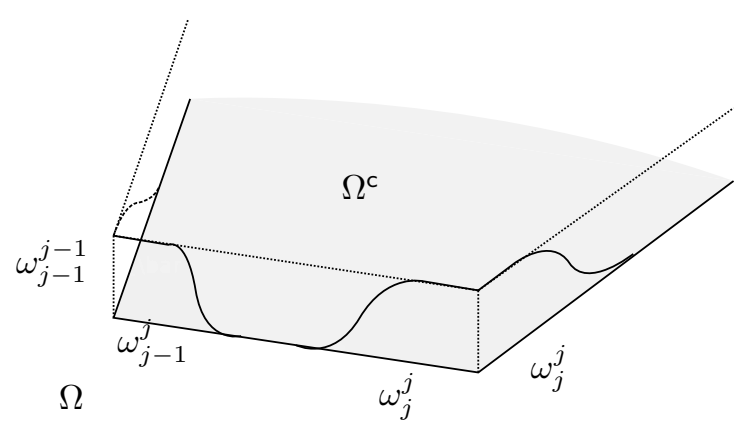

Figure 3.3: The cut-off functions on at a segment of the boundary

\subsection{Weighted Spaces with Asymptotics}

In the following three subsections we will introduce all necessary function spaces which we will need both on the boundary and in the exterior and interior domains. We still require basic knowledge of standard Sobolev spaces, e.g., the spaces $H^{s}\left(\mathbb{R}^{n}\right), H_{0}^{s}(\Omega)$ for bounded domains $\Omega \subset \mathbb{R}^{n}$, Sobolev spaces of compact support, and localized Sobolev distributions.

For the exterior domain, we will define Sobolev spaces which have weighs and asymptotics in a neighborhood of the vertices of the obstacle. Away from the vertices, they will be based on the standard Sobolev spaces mentioned above. Only in chapter 4, we will add the radiation condition to these spaces in order to obtain the proper solution spaces for our scattering problem.

The Sobolev spaces on the boundary will be defined as the weighed Sobolev space on each edge. We choose the weights $\gamma \in \mathbb{R}$ to be the same at the ends of each segment. By introducing asymptotics and requiring compatibility conditions at each vertex, we define the ordinary Sobolev spaces $H^{s}(\partial \Omega)$ on the boundary.

An extremely detailed account for function spaces in the context of Mellin pseudodifferential operators can be found in [RS89], chapter 1. We encourage the reader to delve into this treatise since it offers a complete overview on this subject.

\subsubsection{Sobolev Spaces with Asymptotics in a Cone}

The Mellin transformation has the same pivotal role in singular analysis as the Fourier transformation has in the standard pseudodifferential calculus. It maps functions defined on the positive real axis into the space of meomorphic functions on the complex plane. The basic properties of the Mellin transformation may be found in, e.g., the monographs [Sch91] and [Sch95]. We begin with an important definition: For $\beta \in \mathbb{R}$ we define the parallel lines to the imaginary axis by:

$$
\Gamma_{\beta}:=\{z \in \mathbb{C} \mid \mathfrak{R e}(z)=\beta\} .
$$

Let $u(r) \in C_{c}^{\infty}\left(\mathbb{R}_{+}\right)$. The Mellin transformation is then initially defined by

$$
\mathcal{M}_{0} u(z)=\int_{0}^{\infty} r^{z-1} u(r) d r
$$


It may then be extended to the isomorphism

$$
\mathcal{M}_{0}: L^{2}\left(\mathbb{R}_{+}\right) \stackrel{\cong}{\longrightarrow} L^{2}\left(\Gamma_{\frac{1}{2}}\right) .
$$

For $\gamma \in \mathbb{R}$, set $\mathcal{M}_{\gamma} f(z):=\mathcal{M}\left(r^{\gamma} f\right)(z)$. The Mellin transformation with weight $\gamma$ serves as an isomorphism

$$
\mathcal{M}_{\gamma}: r^{\gamma} L^{2}\left(\mathbb{R}_{+}\right) \stackrel{\cong}{\longrightarrow} L^{2}\left(\Gamma_{\frac{1}{2}-\gamma}\right) .
$$

The inverse of the Mellin transformation is then given by

$$
\left(\mathcal{M}_{\gamma}^{-1} g\right)(r)=\frac{1}{2 \pi i} \int_{\Gamma_{\frac{1}{2}-\gamma}} r^{-z} g(z) d z
$$

where $g \in L^{2}\left(\Gamma_{\frac{1}{2}-\gamma}\right)$. Important identities are

$$
\mathcal{M}\left(r^{\beta} u\right)(z)=(\mathcal{M} u)(z+\beta)
$$

and

$$
\mathcal{M}\left(\left(-r \partial_{r}\right) u\right)=z \mathcal{M} u(z)
$$

Definition 3.2. Let $X$ be a n-dimensional, smooth manifold with boundary. Define the cone $X^{\wedge}$ with base space $X$ by $\mathbb{R}_{+} \times X$. We define the space $\mathcal{H}^{s, \gamma}\left(X^{\wedge}\right)$ as the completion of $C_{c}^{\infty}\left(\mathbb{R}_{+} \times X\right)$ with respect to the norm

$$
\|u\|_{s, \gamma}^{2}=\frac{1}{2 \pi i} \int_{\Gamma_{\frac{n+1}{2}-\gamma}} \int_{\mathbb{R}^{n}}(1+|z|+|\xi|)^{s}\left|\mathcal{M}_{\frac{n}{2}-\gamma} \mathcal{F}(\varphi u)(z, \xi)\right|^{2} d \xi d z .
$$

where the functions $\phi_{j}, j=1, \ldots, N$, are a partition of unity of $X$, and the space

$$
\mathcal{K}^{s, \gamma}\left(X^{\wedge}\right)=\omega \mathcal{H}^{s, \gamma}\left(X^{\wedge}\right)+(1-\omega) H^{s}\left(X^{\wedge}\right)
$$

for a cut-off function $\omega \in C_{c}^{\infty}\left(\overline{\mathbb{R}}_{+}\right)$near the origin and $H^{s}\left(X^{\wedge}\right):=\left.H^{s}(\mathbb{R} \times X)\right|_{\mathbb{R}_{+} \times X}$.

Note that the Mellin transformation yields an isometric isomorphism of the form:

$$
(u, v)_{\mathcal{H}^{0,0}\left(X^{\wedge}\right)}=\frac{1}{2 \pi i} \int_{\Gamma_{\frac{n+1}{2}}}(\mathcal{M} u(z), \mathcal{M} v(z))_{L^{2}(X)} d z .
$$

The pair $(\gamma, \Theta), \gamma \in \mathbb{R}$ and $\Theta=(\vartheta, 0],-\infty \leq \vartheta<0$ is called weight data. In order to define weighted Sobolev spaces with asymptotics, we first have to introduce consider the space

$$
\mathcal{K}_{\Theta}^{s, \gamma}\left(X^{\wedge}\right):=\lim _{\epsilon>0} \mathcal{K}^{s, \gamma-\vartheta-\epsilon}\left(X^{\wedge}\right)
$$

Indeed, $\mathcal{K}_{\Theta}^{s, \gamma}\left(X^{\wedge}\right)$ is the subspace of all $u \in \mathcal{K}^{s, \gamma}\left(X^{\wedge}\right)$ such that for an arbitrary cut-off function $\omega(r)$ the Mellin transform

$$
h(z):=\mathcal{M}_{\gamma-\frac{n}{2}}(\omega u)(z)
$$

has the following two properties: 
1. $h(z) \in \mathcal{A}\left(\left\{z \in \mathbb{C} \mid \frac{n+1}{2}-\gamma-\vartheta<\mathfrak{R e}(z)\right\}\right)$,

2. for $h_{\beta}:=h(\beta+i \rho)$ and any coordinate neighborhood on $X$ with associated cut-off function $\phi$,

$$
\mathcal{F}_{\rho \rightarrow t}^{-1} h_{\beta}(t) \phi(x) \in H^{s}\left(\mathbb{R}_{t, x}^{n+1}\right)
$$

uniformly in $c \leq \beta \leq c^{\prime}$ for every $\frac{n+1}{2}-\gamma+\vartheta<c<c^{\prime}<\infty$.

After this characterization, we introduce the asymptotic types:

Definition 3.3. A discrete asymptotic type $P$ associated with the weight data $(\gamma, \Theta)$ is a finite set of triples

$$
P=\left\{\left(p_{j}, m_{j}, L_{j}\right) \mid p_{j} \in \mathbb{C}, m_{j} \in \mathbb{N}_{0}, j=1, \ldots, N\right\}
$$

such that $L_{j} \subset C^{\infty}(X)$ a finite-dimensional subspace, and

$$
\pi_{\mathbb{C}} P \subset\left\{z \in \mathbb{C} \mid \frac{n+1}{2}-\gamma+\vartheta<\mathfrak{R e}(z)<\frac{n+1}{2}-\gamma\right\} .
$$

To a given asymptotic type $P$ and a fixed cut-off function $\omega$ we attach the set of singular functions given by

$$
\omega_{p_{j}, k_{j}}(t):=t^{-p_{j}} \ln ^{k}(t) \omega(t) l_{j} \quad j \in \mathbb{N}, 0 \leq k \leq m_{j}, l_{j} \in L_{j} .
$$

The Mellin transforms $\mathcal{M}\left(\omega_{p, k}\right)$ of these functions are meromorphic in the complex plane. They are of the form $(z-p)^{-k-1} h(z)$ where $h$ is entire and decreases rapidly along lines parallel to the imaginary axis.

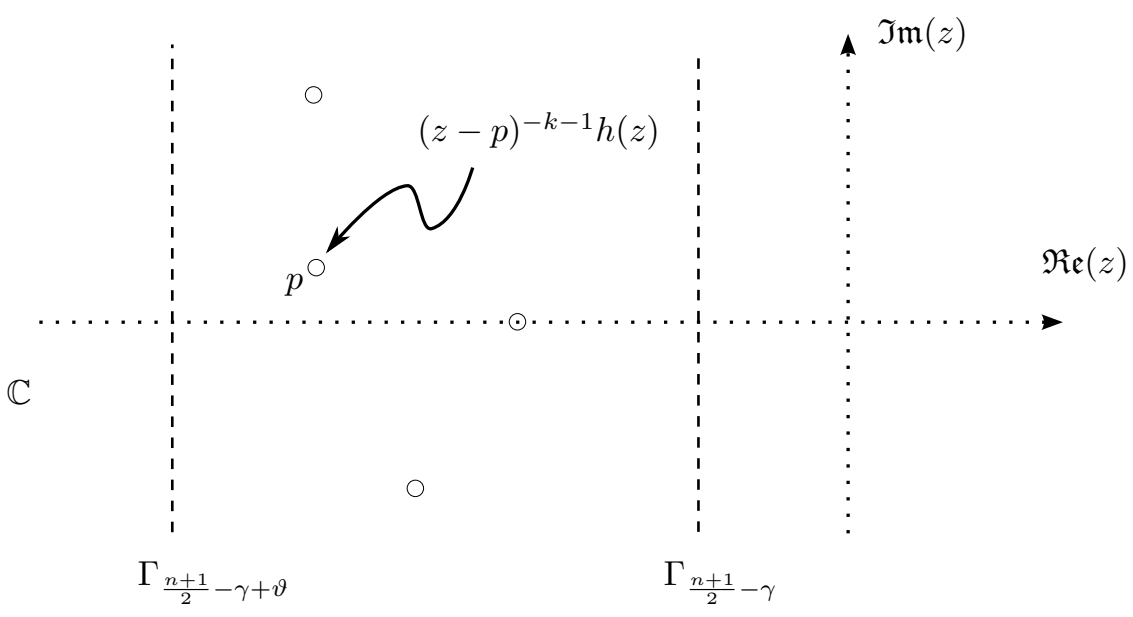

Figure 3.4: Poles $p \in \mathbb{C}$ of an asymptotic type associated with $(\gamma, \Theta)$

Definition 3.4. Let $P$ be an asymptotic type. Then we define the space $\mathcal{E}_{P}\left(\mathbb{R}_{+}\right)$ as the linear span of the functions $\omega_{p, k}$, where $(p, k) \in P$.

Definition 3.5. We define the weighted Sobolev space with asymptotics as

$$
\mathcal{K}_{P}^{s, \gamma}\left(X^{\wedge}\right):=\mathcal{K}_{\Theta}^{s, \gamma}\left(X^{\wedge}\right)+\mathcal{E}_{P}^{s, \gamma}\left(X^{\wedge}\right) .
$$


Note that the space $\mathcal{E}_{P}^{s, \gamma}\left(X^{\wedge}\right)$ has the topology of a finite dimensional vector space. We proceed with giving examples of spaces with asymptotics for the one dimensional case (i.e. $X=\{\mathrm{pt}\}$ ):

Example 3.6. For $s \in \mathbb{R}$, define the number

$$
\kappa(s):=\{k \in \mathbb{N}|k<| s \mid-1 / 2\} .
$$

Set for $s>1 / 2$

$$
\begin{gathered}
\mathcal{T}:=\left\{(-j, 0) \mid j \in \mathbb{N}_{0}\right\}, \\
\mathcal{T}^{s}:=\{(-j, 0) \mid j=0,1, \ldots, \kappa(s)\} .
\end{gathered}
$$

Both these asymptotic types describe Taylor asymptotics. The corresponding spaces are

$$
\mathcal{E}_{\mathcal{T}^{s}}\left(\mathbb{R}_{+}\right):=\operatorname{span}\left\{t^{j} \mid j \in \mathbb{N}_{0}\right\}
$$

and

$$
\mathcal{E}_{\mathcal{T}^{s}}\left(\mathbb{R}_{+}\right):=\operatorname{span}\left\{t^{j} \mid j=0,1, \ldots, \kappa(s)\right\} .
$$

Furthermore, for the following theorem, we define $(s<-1 / 2)$ :

$$
\mathcal{E}_{\mathcal{D}^{s}}\left(\mathbb{R}_{+}\right):=\operatorname{span}\left\{\frac{d^{j}}{d t^{j}} \delta_{0} \mid j=0,1, \ldots, \kappa(s)\right\},
$$

where $\delta_{0}$ is the Dirac delta function with $\operatorname{supp} \delta_{0}=\{0\}$. Notice that we have $\mathcal{E}_{\mathcal{P}}\left(\mathbb{R}_{+}\right) \subset \mathcal{K}^{\infty, \gamma}\left(\mathbb{R}_{+}\right)$.

Theorem 3.7. Let $s \in \mathbb{R}$, then there are canonical isomorphisms

$$
H^{s}\left(\mathbb{R}_{+}\right) \cong \begin{cases}\mathcal{K}^{s, s}\left(\mathbb{R}_{+}\right)+\mathcal{E}_{\mathcal{T} s}\left(\mathbb{R}_{+}\right) & \text {for } s \geq 0, s \neq \frac{1}{2} \bmod \mathbb{Z}, \\ \mathcal{K}^{s, s}\left(\mathbb{R}_{+}\right) & \text {for } s \leq 0,\end{cases}
$$

and

$$
H_{0}^{s}\left(\overline{\mathbb{R}}_{+}\right) \cong \begin{cases}\mathcal{K}^{s, s}\left(\mathbb{R}_{+}\right) & \text {for } s \geq 0, \\ \mathcal{K}^{s, s}\left(\mathbb{R}_{+}\right)+\mathcal{E}_{\mathcal{D}^{s}}\left(\mathbb{R}_{+}\right) & \text {for } s \leq 0, s \neq \frac{1}{2} \bmod \mathbb{Z}\end{cases}
$$

The isomorphism for $H^{s}\left(\mathbb{R}_{+}\right)$follows by identifying distributions on $\mathbb{R}_{+}$and that for $H_{0}^{s}\left(\overline{\mathbb{R}}_{+}\right)$by duality. The identifications are continuous in both directions.

Of course, there is the possibility to define the weighted Sobolev space with different weights at each end of the edge $\partial \Omega_{j}$. However, this is not necessary for the problem of this thesis.

Notice also, that in the interior of the edge the space $\mathcal{H}^{s, \gamma}\left(\partial \Omega_{j}\right)$ is equivalent to an ordinary Sobolev space of order $s \in \mathbb{R}$. In symbols, $\theta_{j} \mathcal{H}^{s, \gamma}\left(\partial \Omega_{j}\right) \cong \theta_{j} H^{s}(\mathbb{R})$ and $\|u\|_{s, \gamma}=\|u\|_{s}$ for $u \in \mathcal{H}^{s, \gamma}\left(\partial \Omega_{j}\right)$ with support bounded away from the endpoints.

Definition 3.8. A function $\chi \in C^{\infty}(\mathbb{C})$ is called an $A$-excision function for some $A \subset \mathbb{C}$, if $0 \leq \chi \leq 1, \chi(z)=0$ for all $z$ in an open neighbourhood of $\bar{A}$, $\chi(z)=1$ outside another open neighborhood of $\bar{A}$. 
So far, we have only dealt with weighted spaces with finite asymptotics. Defining spaces with infinite asymptotics does not pose a great challenge, though.

Let $\left\{P_{l} \mid l \in \mathbb{N}\right\}$, be a sequence of discrete asymptotic types with $P_{l} \subseteq P_{l+1}$ for all $l$. Then, using the continuous embeddings $\mathcal{K}_{P_{l+1}}^{s, \gamma}\left(X^{\wedge}\right) \hookrightarrow \mathcal{K}_{P_{l}}^{s, \gamma}\left(X^{\wedge}\right)$ for all $l \geq 0$, we may endow the resulting space with a Fréchet topology:

Definition 3.9. We define the weighted space with infinite asymptotics $P$ by the projective limit

$$
\mathcal{K}_{P}^{s, \gamma}\left(X^{\wedge}\right)=\lim _{i \in \mathbb{N}} \mathcal{K}_{P_{l}}^{s, \gamma}\left(X^{\wedge}\right)
$$

\subsubsection{Sobolev Spaces on the Boundary}

Since the boundary $\partial \Omega$ is not smooth but polygonal, the definition of the space $H^{s}(\partial \Omega)$ needs some more preparation. The usual way to define Sobolev spaces on a hypersurface is to define them as the traces of Sobolev spaces in the surrounding space. But since we are working with Mellin pseudodifferential operators on the boundary, we need to take the technically more cumbersome route and define them intrinsically via the Mellin transform.

For $m \in \mathbb{N}_{0}$, define the Sobolev space with weight $\gamma \in \mathbb{R}$ on the positive real axis as

$$
\mathcal{H}^{m, \gamma}\left(\mathbb{R}_{+}\right):=\left\{u \in L^{2}\left(\mathbb{R}_{+}\right) \mid\left(-r \frac{d}{d r}\right)^{j} u \in L^{2}\left(\mathbb{R}_{+}\right), j=0,1, \ldots, m\right\} .
$$

Definition 3.10. The weighted Sobolev space $\mathcal{H}^{s, \gamma}\left(\mathbb{R}_{+}\right), s, \gamma \in \mathbb{R}$ is defined as the closure of $C_{0}^{\infty}\left(\mathbb{R}_{+}\right)$with respect to the norm

$$
\|u\|_{s, \gamma}=\sqrt{\frac{1}{2 \pi i} \int_{\Gamma_{\frac{1}{2}-\gamma}}\langle\mathfrak{I m}(z)\rangle^{2 s}\left|\mathcal{M}_{\gamma} u(z)\right|^{2}} d z,
$$

where $\langle\xi\rangle=\sqrt{1+|\xi|^{2}}$ is the usual Japanese bracket.

Some easy consequences of this definition are, for example,

$$
\mathcal{H}^{s, \gamma}\left(\mathbb{R}_{+}\right)=t^{\gamma} \mathcal{H}^{s, 0}\left(\mathbb{R}_{+}\right)=t^{\gamma} \mathcal{H}^{s}\left(\mathbb{R}_{+}\right)
$$

and

$$
\mathcal{K}^{s, 0}\left(\partial \Omega_{j}\right)=H^{s}\left(\partial \Omega_{j}\right)
$$

The analogue of the Rellich's lemma does also exist for weighted Sobolev spaces:

Proposition 3.11. Let $a>0$. Then the embedding

$$
\mathcal{H}^{s^{\prime}, \gamma^{\prime}}([0, a)) \hookrightarrow \mathcal{H}^{s, \gamma}([0, a)),
$$

is compact if and only if $s^{\prime}>s$ and $\gamma^{\prime}>\gamma$.

Hence, one needs improvement in the decay at the origin in order to obtain compactness. As we will see in theorem 6.10 , this is the reason why the double layer potential fails to be compact on the boundary of a polygon. ${ }^{1}$

\footnotetext{
${ }^{1}$ This fact has been mentioned in [OP04] for the context of weighted Sobolev spaces without any proof or calculation.
} 
Consider the parametrization $x_{j}:\left(0, l_{j}\right) \rightarrow \partial \Omega_{j}$ and let $\omega_{1}$ and $\omega_{2}$ are cutoff functions in $C_{c}^{\infty}\left(\overline{\mathbb{R}}_{+}\right)$with $\omega_{1}$ supported in a small neighborhood of $0, \omega_{2}$ supported in a small neighborhood of $l_{j}$. Then set $\mathcal{H}^{s, \gamma}\left(\left(0, l_{j}\right)\right):=\omega_{1} \mathcal{H}^{s, \gamma}\left(\mathbb{R}_{+}\right) \oplus$ $\omega_{2} \mathcal{H}^{s, \gamma}\left(\left(-\infty, l_{j}\right)\right)$, where the latter space is defined in the obvious way.

Definition 3.12. For $s, \gamma \in \mathbb{R}$, define the Sobolev space on the edge $\partial \Omega_{j}$ of regularity $s$ and weight $\gamma$ as

$$
\mathcal{H}^{s, \gamma}\left(\partial \Omega_{j}\right):=x_{j}^{*} \mathcal{H}^{s, \gamma}\left(\left(0, l_{j}\right)\right) .
$$

The norm of this space is given by

$$
\|u\|_{s, \gamma}^{2}:=\left\|x_{j}^{*} \omega_{1} u\right\|_{s, \gamma}^{2}+\left\|x_{j}^{*} \omega_{2} u\right\|_{s, \gamma}^{2} .
$$

Note that $C_{c}^{\infty}\left(\partial \Omega_{j}\right)$ is dense in $\mathcal{H}^{s, \gamma}\left(\partial \Omega_{j}\right)$ with repect to this topology.

Definition 3.13. Let $s, \gamma \in \mathbb{R}$. We define the space of weighed Sobolev functions on the boundary $\partial \Omega$ with weight $\gamma$ and regularity $s$ as the direct sum

$$
\mathcal{H}^{s, \gamma}(\partial \Omega):=\bigoplus_{j=1}^{N} \mathcal{H}^{s, \gamma}\left(\partial \Omega_{j}\right)
$$

Of course, we could have defined the function space with different weights and regularities for each edge of the boundaries. But for our purposes, it suffices to stick to global parameters $s$ and $\gamma$. The norm and topology for $\mathcal{H}^{s, \gamma}(\partial \Omega)$ is defined in the obvious way.

Definition 3.14. Let $m \in \mathbb{N}_{0}$. Then we define

$$
H^{m}(\partial \Omega):=\left\{\left(f_{j}\right)_{j=1}^{N} \in \prod_{j=1}^{N} H^{m}\left(\partial \Omega_{j}\right) \mid\left(f_{j}\right)_{j=1}^{N} \text { satisfy condition } 3.9\right\},
$$

where condition is

$$
f_{j}^{(l)}\left(v_{j}\right)=f_{j+1}^{(l)}\left(v_{j}\right) \quad \forall v_{j} \quad \forall l=0,1, \ldots, m-1 .
$$

We define the corresponding norm simply as

$$
\|u\|_{k}=\sum_{j=1}^{N}\left\|u_{j}\right\|_{k}
$$

For $s>0, H^{-s}(\partial \Omega)$ will be defined as the dual to $H^{s}(\partial \Omega)$.

The space $\mathcal{H}^{s, \gamma}(\partial \Omega)$ is a natural space on which our calculus of Mellin pseudodifferential operators can be defined on in section 4.3.

\subsubsection{Sobolev Spaces in the Exterior Domain}

The standard Sobolev spaces $H^{s}\left(\mathbb{R}^{n}\right)$ and $H^{s}(\Omega), \Omega \subseteq \mathbb{R}^{n}$ shall be defined in the ordinary fashion. It will be our last task to define the spaces in the exterior domain with weights and asymptotics. 


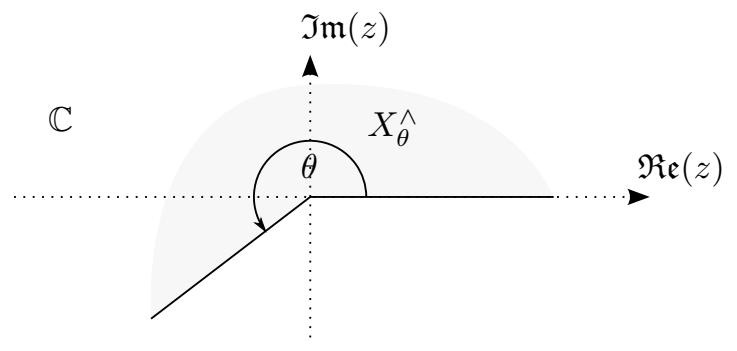

Figure 3.5: The sector $X_{\theta}^{\wedge}$

In other words, a neighborhood of the corner exterior domain is mapped onto the sector $X_{\theta}^{\wedge}, 0<\theta<2 \pi$, defined by

$$
X_{\theta}^{\wedge}:=\left\{r e^{i \varphi} \in \mathbb{C} \mid r>0,0<\varphi<\theta\right\} .
$$

If we set $X:=(0, \theta)$, then actually $X_{\theta}^{\wedge} \cong \mathbb{R}_{+} \times(0, \theta)$. In order to define a weighted Sobolev space for the exterior domain of a polygonal obstacle, let $\Upsilon_{j}$ : $U_{j} \rightarrow V_{j}$ be the diffeomorphism which maps the vicinity of the vertex $v_{j}$ onto a neighborhood of the origin of the sector $X_{2 \pi-\alpha_{j}}^{\wedge}$. Since we are assuming that the edges are straight in a neighbourhood of each vertex, this diffeomorphism will be of the form ${ }^{2}$

$$
\Upsilon_{j}(z):=e^{i \theta_{j}} x-v_{j},
$$

where $0 \leq \theta_{j}<2 \pi, v_{j} \in \mathbb{R}^{2}$, where $v_{j} \in \mathbb{R}^{2}$ is being used as the vertex' coordinate and $\theta_{j} \in[0 ; 2 \pi)$ an appropriate angle. $\Upsilon_{j}$ maps a neighborhood of the corner onto $V_{j}$ such that $v_{j} \in U_{j}, \Psi_{j}\left(v_{j}\right)=0, \Upsilon_{j}\left(\partial \Omega_{j}\right)=V_{j} \cap\left(e^{-i \alpha_{j}} \mathbb{R}_{+}\right)$, and $\Upsilon_{j}\left(U_{j} \cap \partial \Omega_{j+1}\right)=V_{j} \cap \mathbb{R}$.

We then define the weighted Sobolev space for the exterior domain:

Definition 3.15. For $s, \gamma \in \mathbb{R}$ and a general exterior domain of an obstacle fulfilling the straight-edge condition, we define the space

$$
\mathcal{K}^{s, \gamma}(\Omega):=\sum_{j=1}^{N} \Phi_{j} \Upsilon_{j}^{*} \mathcal{H}^{s, \gamma}\left(X_{2 \pi-\alpha_{j}}^{\wedge}\right)+\left(1-\sum_{j=1}^{N} \Phi_{j}\right) H_{\mathrm{loc}}^{s}(\Omega) .
$$

Of course, it will be necessary to equip these spaces with asymptotics. Since this is done in a straightforward way, we will omit the construction here.

Remark 3.16. Throughout this thesis we will make extensive use of the above coordinate transformation. However, in order to keep the notation simple, we will ignore mentioning them explicitly in any way.

\subsection{The Mellin Calculus on $\mathbb{R}_{+}$}

Having defined the necessary function spaces, the next step will be to define the calculus of operators on the boundary $\partial \Omega$. Again, we will first define them on $\mathbb{R}_{+}$, and only afterwards define the whole algebra on the boundary which

\footnotetext{
${ }^{2}$ Abusing the letter $v_{j}$ both as denotation of a vertex and as a coordinate $v_{j} \in \mathbb{R}^{2}$.
} 
is essentially a closed graph with valence number two. While standard pseudodifferential calculus will still play a role in the interior of the edges, Mellin pseudodifferential operators will be the appropriate substitution at the ends of each edge. Like pseudodifferential operators, Mellin pseudodifferential operators come in different flavours through the use of different symbols.

\subsubsection{Mellin Pseudodifferential Operators}

Let $X$ be a closed $C^{\infty}$-manifold. By $L^{m}\left(X ; \Gamma_{\beta}\right)$ we shall denote the space of parameter-dependent pseudodifferential operators on $X$. However, as we will only encounter degenerate base spaces (i.e. $X=\{\mathrm{pt}\}$ ), these spaces reduce to $L^{m}\left(X ; \Gamma_{\beta}\right)=S^{m}\left(\Gamma_{\beta}\right)$.

Before we continue to define the symbol spaces, recall the definition of excision functions (Definition 3.8). We are now in a position to define the spaces of Mellin symbols:

Definition 3.17 (Mellin symbols).

1. Let $P$ be an asymptotic type, $m \in \mathbb{R}$. The space of meromorphic symbols of order $m$ and asymptotic type $P, M_{P}^{m}$, is defined as the set of meromorphic functions $h(z)$ on $\mathbb{C}$ with poles at $z=p_{j}$ of order $m_{j}+1$. The principal part of the Laurent expansion of $h(z)$ at $z=p_{j}$ is $\sum_{k=0}^{m_{j}} d_{j k}\left(z-p_{j}\right)^{-k}$, $d_{j k} \in \mathbb{C}$ for $0 \leq k \leq m_{j}$.

For a $\pi_{\mathbb{C}} P$-excision function $\chi \in C^{\infty}(\mathbb{C})$ and $\beta_{0}<\beta_{1} \in \mathbb{R}$ arbitrary, we have $\left.\chi(z) h(z)\right|_{\Gamma_{\beta}} \in S^{m}\left(\Gamma_{\beta}\right)$ uniformly for $\beta \in\left[\beta_{0} ; \beta_{1}\right]$.

2. The space of holomorphic symbols of order $m, M_{O}^{m}$, is defined as the space of all holomorphic functions $h(z) \in \mathcal{A}(\mathbb{C})$ such that $\left.h\right|_{\Gamma_{\beta}} \in S^{m}\left(\Gamma_{\beta}\right)$ uniformly for $\beta \in\left[\beta_{0} ; \beta_{1}\right], \beta_{0}<\beta_{1} \in \mathbb{R}$.

3. Finally, $M_{P}^{-\infty}$ shall be the space of all functions $h(z) \in \mathcal{A}\left(\mathbb{C} \backslash \pi_{\mathbb{C}} P ; S^{-\infty}\right)$ which are rapidly decreasing along $\Gamma_{\beta}$ with $\Gamma_{\beta} \cap \pi_{\mathbb{C}} P=\emptyset$.

Remark 3.18. Note that a decomposition of the Mellin symbols of the form

$$
M_{P}^{m}=M_{O}^{m}+M_{R}^{-\infty}
$$

as it is usual for non-trivial base spaces is not necessary.

Definition 3.19. Let $\gamma \in \mathbb{R}$ and $f$ be a holomorphic function. We denote the corresponding shift operator by $\tau^{\gamma}$. That is,

$$
\tau^{\gamma} f(z):=f(z+\gamma)
$$

together with an appropriate shift of the domain.

Definition 3.20 (Mellin pseudodifferential operators). An operator

$$
f \mapsto t^{\delta-\gamma} \omega \mathrm{op}_{\mathrm{M}}\left[\tau^{\gamma} h\right]\left(t^{\gamma} \omega f\right)
$$

with $\omega$ a cut-off function at zero, $h$ a Mellin symbol, and $\mu \in \mathbb{R}$, is called a Mellin operator of conormal order $\delta$. 
The natural domain of Mellin pseudodifferential operators are the weighted Sobolev spaces $\mathcal{H}^{s, \gamma}\left(\mathbb{R}_{+}\right)$.

Proposition 3.21. Let $A$ be a Mellin pseudodifferential operator of order $m$ and conormal order $\rho$. Let $h \in M_{P}$ be the Mellin symbol. Then $A$ can be expended to a continuous operator

$$
\mathrm{A}: \mathcal{H}^{s, \gamma}\left(\mathbb{R}_{+}\right) \rightarrow \mathcal{H}^{s-m, \gamma-\rho}\left(\mathbb{R}_{+}\right) .
$$

Furthermore, if $P$ is the asymptotic type, then $\mathrm{A}$ induces

$$
\mathrm{A}: \mathcal{H}^{s, \gamma}\left(\mathbb{R}_{+}\right) \rightarrow \mathcal{H}_{P}^{s-m, \gamma-\rho}\left(\mathbb{R}_{+}\right) .
$$

Later, we will have to deal with the behaviour of functions with asymptotics under the application of a Mellin pseudodifferential operator. According to Lemma 3.24, this basically amounts to the multiplication of two meromorphic functions. Let $h_{P_{i}} \in \mathcal{A}\left(\mathbb{C} \backslash \pi_{\mathbb{C}} P_{i}\right), i=1,2, P_{i}=\left\{\left(p_{j}, m_{j}\right)\right\}_{j \in \mathbb{Z}}$ be meromorphic with poles at $p_{j}$ of multiplicity $m_{j}+1$, and $h_{P_{i}}$ nonzero on the complement of $\pi_{\mathbb{C}} P_{i}$. Then the poles of the product $h_{P_{1}} h_{P_{2}}$ are of type $P_{1}+P_{2}$ which is defined as follows:

Definition 3.22. Let $P_{1}, P_{2}$ be two asymptotic types as defined above. Denote by $P_{1} \dot{+} P_{2}$ the set which is characterized by

$$
\pi_{\mathbb{C}}\left(P_{1} \dot{+} P_{2}\right)=\pi_{\mathbb{C}} P_{1} \cup \pi_{\mathbb{C}} P_{2}
$$

and

$$
(p, m) \dot{+}(q, n)= \begin{cases}(p, m) \cup(q, n) & \text { when } p \neq q, \\ (p, m+n+1) & \text { when } p=q .\end{cases}
$$

Using this definition, we can tell something about the transformation of asymptotic types under the mapping of Mellin pseudodifferential operators. The proofs are straightforward.

Proposition 3.23. Let $P, Q$ be two asymptotic types. Let $h \in M_{Q}^{m}, u \in$ $\mathcal{H}_{P}^{s, \gamma}\left(\mathbb{R}_{+}\right)$. Then

$$
\mathrm{op}_{\mathrm{M}}^{\gamma} h: \mathcal{H}_{P}^{s, \gamma}\left(\mathbb{R}_{+}\right) \rightarrow \mathcal{H}_{P \dot{+} Q}^{s-m, \gamma}\left(\mathbb{R}_{+}\right) .
$$

Lemma 3.24. Let $h_{1}$ and $h_{2}$ be two Mellin symbols with $h_{i} \in M_{P_{i}}, i=1,2$, with two asymptotic types $P_{i}$. Then $h_{1} h_{2} \in M_{P_{1}+P_{2}}^{m_{1}+m_{2}}$ and

$$
\mathrm{op}_{\mathrm{M}}^{\gamma}\left[h_{1} h_{2}\right]=\mathrm{op}_{\mathrm{M}}^{\gamma}\left[h_{1}\right] \mathrm{op}_{\mathrm{M}}^{\gamma}\left[h_{2}\right] .
$$

In addition,

$$
\mathrm{op}_{\mathrm{M}}^{\gamma}\left[h_{1} h_{2}\right]: \mathcal{H}^{s, \gamma}\left(\mathbb{R}_{+}\right) \rightarrow \mathcal{H}_{P_{1}+P_{2}}^{s, \gamma-m_{1}-m_{2}}\left(\mathbb{R}_{+}\right) .
$$

The following example illustrates how Mellin pseudodifferential opertors occur naturally:

Example 3.25. Let $u$ and $f$ be in $C_{c}^{\infty}\left(\mathbb{R}_{+}\right)$. Consider the (multiplicative) convolution operator on $\mathbb{R}_{+}$:

$$
(f * u)(r):=\int_{0}^{\infty} f\left(\frac{t}{s}\right) u(s) \frac{d s}{s},
$$


where $*$ denotes the convolution with respect to the multiplicative group $\left(\mathbb{R}_{+}, \cdot\right)$. We can express this operator in the Mellin image by utilizing Plancherel's theorem, i.e. calculating

$$
\begin{aligned}
\mathcal{M}_{\gamma}[f * u](z) & =\int_{0}^{\infty} t^{z+\gamma-1}\left[\int_{0}^{\infty} f\left(\frac{t}{s}\right) u(s) \frac{d s}{s}\right] d t \\
& =\int_{0}^{\infty} s^{z+\gamma-1} f(s)\left[\int_{0}^{\infty}\left(\frac{t}{s}\right)^{z+\gamma-1} u(s) d t\right] \frac{d s}{s} \\
& =\mathcal{M}_{\gamma} f(z) \cdot \mathcal{M}_{\gamma} u(z) .
\end{aligned}
$$

Hence, setting $h_{\gamma}(z):=\mathcal{M}_{\gamma} f(z)$, the operator may be written as

$$
\mathrm{op}_{\mathrm{M}}^{\gamma}[h] u(r):=\mathcal{M}_{\gamma}^{-1}\left[h_{\gamma}(z) \cdot \mathcal{M}_{\gamma}[u](z)\right](r) .
$$

\subsubsection{Conormal Symbols and Ellipticity on $\mathbb{R}_{+}$}

Having settled the definition of Mellin operators in the previous section, we may start to define the calculus of Mellin operators on $\mathbb{R}_{+}$. Defining the calculus will include intruducing the so-called Green operators $G$ and the smoothing Mellin operators $M$. We will define the space $C_{\mathrm{G}}\left(\mathbb{R}_{+}, \mathbf{g}\right), C_{\mathrm{M}+\mathrm{G}}\left(\mathbb{R}_{+}\right)$, and finally the operator algebra $C^{m}\left(\mathbb{R}_{+}, \mathbf{g}\right)$. The cone algebra on $\mathbb{R}_{+}$with discrete asymptotics is a certain subspace of $\left\{t^{\delta} \mathrm{op}_{\mathrm{M}}(f) t^{-\gamma} \mid f\left(t, t^{\prime}, z\right) \in S_{\mathrm{cl}}^{\mu}\left(\overline{\mathbb{R}}_{+} \times \overline{\mathbb{R}}_{+} \times \Gamma_{\frac{1}{2}}\right)\right\}, \gamma \in \mathbb{R}$, and some weights $\gamma, \delta \in \mathbb{R}$.

Theorem 3.26. If $\mathrm{A}$ is an elliptic operator of order $m$ on a compact graph with respect to a weight factor $\gamma$, then there is a singularity type $P$ such that the operator

$$
\mathrm{A}: \mathcal{H}^{s, \gamma}\left(\mathbb{R}_{+}\right) \rightarrow \mathcal{H}_{P}^{s-m, \gamma-m}\left(\mathbb{R}_{+}\right)
$$

defines a Fredholm map.

Definition 3.27 (Green operator). Denote by

$$
\mathcal{C}_{\mathrm{G}}(X, \mathbf{g})_{P, Q}
$$

the subspace of Green operators with fixed asymptotic types $P, Q$. Then is a Fréchet space in a natural way if we use the canonical Fréchet topologies of An operator $\mathrm{G} \in L^{-\infty}\left(\mathbb{R}_{+}\right)$inducing continuous maps

$$
\begin{gathered}
\mathrm{G}: \mathcal{K}^{s, \gamma}\left(\mathbb{R}_{+}\right) \rightarrow \mathcal{S}_{P}^{\delta}\left(\mathbb{R}_{+}\right), \\
\mathrm{G}^{*}: \mathcal{K}^{s,-\delta}\left(\mathbb{R}_{+}\right) \rightarrow \mathcal{S}_{Q}^{-\gamma}\left(\mathbb{R}_{+}\right)
\end{gathered}
$$

for all $s \in \mathbb{R}$ and discrete asymptotic types $P$ and $Q$ with weight data $(\delta, \Theta)$ and $(-\gamma, \Theta)$, respectively, is called a Green operator with discrete asymptotics. The space of these operators is denoted by $\mathcal{C}_{\mathrm{G}}\left(\mathbb{R}_{+},(\gamma, \delta, \Theta)\right)$. Here,

$$
\mathcal{S}_{P}^{\gamma}\left(\mathbb{R}_{+}\right):=\left\{\omega u+(1-\omega) v \mid u \in \mathcal{K}_{P}^{\infty, \gamma}\left(\mathbb{R}_{+}\right), v \in \mathcal{S}\left(\overline{\mathbb{R}}_{+}\right)\right\},
$$

with $\mathcal{S}\left(\overline{\mathbb{R}}_{+}\right):=\left.\mathcal{S}(\mathbb{R})\right|_{\mathbb{R}_{+}}$. 
Definition 3.28 (Smoothing Mellin operator). Smoothing Mellin operators will be defined as finite linear combinations of operators of the form

$$
\mathrm{A}_{j}=r^{-\mu+j} \omega \mathrm{op}_{\mathrm{M}}^{\alpha_{\mathrm{j}}}\left[h_{j}\right] \omega_{0}, \quad j \in \mathbb{N},
$$

$h_{j} \in M_{R_{j}}^{-\infty}, R_{j}$ an asymptotic type with $\pi_{\mathbb{C}} R_{j} \cap \Gamma_{\frac{1}{2}-\alpha_{j}}$, and $\omega, \omega_{0}$ being arbitrary cut-off functions with $\omega \omega_{0}=\omega_{0}$.

Remark 3.29. Given two operators of the form $\mathrm{A}_{\mathrm{M}}$ with the same $h_{0}, \ldots, h_{k-1}$ but different cut-off functions or shifts, their difference belongs to $\mathcal{C}_{\mathrm{G}}\left(\mathbb{R}_{+},(\gamma, \delta, \Theta)\right)$.

Definition 3.30. Denote by $\mathcal{C}_{\mathrm{M}+\mathrm{G}}(X, g)$ the space of all operators

$$
\mathrm{A}=\mathrm{M}+\mathrm{G} \quad \text { with } \mathrm{M}=\sum_{j=0}^{k-1} \mathrm{~A}_{j}
$$

with arbitrary $\mathrm{A}_{j}$ of the form $\mathrm{A}_{j}=r^{-\mu+j} \omega \mathrm{op}_{\mathrm{M}}^{\alpha_{\mathrm{j}}}\left[h_{j}\right] \omega_{0}$ as above, the $\alpha_{j}$ satisfying $\gamma-j \leq \alpha_{j} \leq \gamma$, and Green operators $\mathrm{G} \in \mathcal{C}_{G}(X, g)$. We set

$$
\sigma_{\mathrm{M}}^{m-j}(\mathrm{M})(z)=h_{j}(z), \quad j=0,1, \ldots, k-1,
$$

called the conormal symbol of A of conormal order $(m-j)$.

Definition 3.31. The space $\mathcal{C}^{\mu}\left(\mathbb{R}_{+}, \mathbf{g}\right)$ of all cone operators of order $\mu \in \mathbb{R}$ on $\mathbb{R}_{+}$with discrete asymptotics and weight data $\mathbf{g}=(\gamma, \delta, \Theta), \gamma, \delta \in \mathbb{R}, \Theta=$ $(-(k+1), 0], k \in \mathbb{N}$, is defined as the set of all operators of the form

$$
\mathrm{A}=\omega t^{\delta-\gamma} \mathrm{op}_{\mathrm{M}}^{\gamma}[h] \omega_{0}+(1-\omega) A\left(1-\omega_{1}\right)+\mathrm{M}+\mathrm{G}
$$

with cut-off functions $\omega, \omega_{0}, \omega_{1}$ satisfying $\omega \omega_{0}=\omega_{0}, \omega \omega_{1}=\omega_{1}$, and

1. $h(t, z) \in C^{\infty}\left(\overline{\mathbb{R}}_{+}, M_{O}^{m}\right)$,

2. $\mathrm{M}+\mathrm{G} \in C_{\mathrm{M}+\mathrm{G}}\left(\mathbb{R}_{+}, \mathbf{g}\right)$,

3. $\mathrm{A} \in L_{\mathrm{cl}}^{\mu}\left(\mathbb{R}_{+}\right) \cap L^{\mu ; 0}\left(\mathbb{R}_{+}\right)$.

An operator $\mathrm{A} \in C^{\mu}\left(\mathbb{R}_{+}, \mathbf{g}\right)$ for $\mathbf{g}=(\gamma, \delta, \Theta)$ induces continuous maps

$$
\mathrm{A}: \mathcal{K}^{s, \gamma}\left(\mathbb{R}_{+}\right) \rightarrow \mathcal{K}^{s-\mu, \delta}\left(\mathbb{R}_{+}\right)
$$

as well as

$$
\mathrm{A}: \mathcal{K}_{P}^{s, \gamma}\left(\mathbb{R}_{+}\right) \rightarrow \mathcal{K}_{Q}^{s-\mu, \delta}\left(\mathbb{R}_{+}\right)
$$

for all $s \in \mathbb{R}$ and any asymptotic type $P \in \operatorname{As}(\gamma, \Theta)$, with some resulting asymptotic type $Q$. Note that we have $C^{\mu}\left(\mathbb{R}_{+}, \mathbf{g}\right) \subset L_{\mathrm{cl}}^{\mu} \cap L^{\mu ; 0}\left(\mathbb{R}_{+}\right)$.

The conormal symbol of $A$ is defined as the operator family

$$
\sigma_{\mathrm{M}}^{m}(\mathrm{~A})(z)=h(0, z)+h_{0}(z),
$$

with $\sigma_{\mathrm{M}}^{m}(\mathrm{~W})(z)$ and $z \in \Gamma_{\frac{1}{2}-\gamma}$. The poles are determined by the asymptotic type $R$ of $h_{0}(z)$, more precisely by $\pi_{\mathbb{C}}(R)$. We also have the conormal symbols of lower orders

$$
\sigma_{\mathrm{M}}^{m-j}(\mathrm{~A})(z)=\frac{1}{j !}\left(\frac{\partial^{j}}{\partial r^{j}} h\right)(0, z)+\sigma_{\mathrm{M}}^{m-j}(\mathrm{M})(z)
$$

for $j=0, \ldots, k-1, \Theta=(-k, 0]$. 
Definition 3.32 (Ellipticity). Let the operator A be of order $m$, i.e. both its interior and conormal orders are equal to $m$. For a real number $\gamma$, we say that a cone-degenerate operator is elliptic with respect to $\gamma$ if

(i) the interior symbol is elliptic in the usual sense,

(ii) the principal conormal symbol of order $m$ has no zeros on the line $\Gamma_{\frac{1}{2}-\gamma}$. 


\section{Chapter 4}

\section{Obstacle Scattering in Terms of Boutet de Monvel's Algebra}

In this chapter, we will recast the scattering problem into the language of Boutet de Monvel's algebra. It is the author's intention to provide a line of thought and some systematization for the uninitiated reader. We will avoid giving a full-blown introduction into the machinery of the algebra.

The last section of this chapter contains a technical definition of the potential operators which are operators occuring in the right upper corner in Boutet de Monvel's algebra. The definitions are not difficult to understand and will come to use in chapter 6 when we are analysing the mapping properties of the layer potentials.

The elements of Boutet de Monvel algebras consist of $2 \times 2$-matrices of operators:

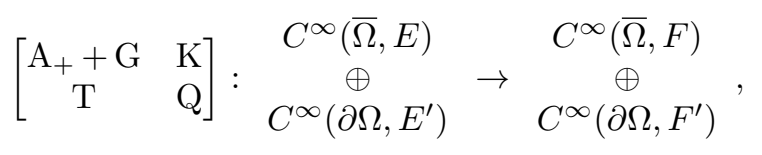

where $E, F$ are finite dimensional vector bundles over $\Omega$, and $E^{\prime}, F^{\prime}$ are finite dimensional vector bundles over $\partial \Omega$, respectively. The matrix consists of four different types of operators which, upon localization, have different local manifestations. We denote the different types of operators as:

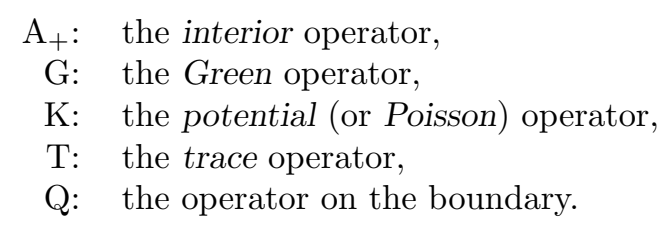

Before we are able to define an algebra for the whole problem, we need to give each of the above classes of operators a local meaning. Of course, it is necessary to distinguish between the situation in the smooth interior of the boundary's edges and in a neighborhood of the vertices. Also, these operators will in general depend on a parameter, in our case, on a complex parameter $\lambda$. 
Again, we will only outline the theory, since a thorough treatment would require too much space. For details, the reader is invited to consult [Gru09] for the theory for smoothly bounded domains, and the two-parted publication Boundary Value Problems and Conical Singularities I + II ([SS94] and [SS95]) for the theory in conical domains. We will mostly focus on the potential and boundary operators and only take a quick glance at the interior operators since the latter will not play large role in subsequent chapters.

Throughout our discussion, we will use a finite open covering of $\Omega$ of the following sort:

Definition 4.1. Let $U_{j} \subset \Omega, j=1, \ldots, N$, be a mutually disjoint set of neighborhoods of the vertices $v_{j} \in U_{j}$. Let $V_{j} \subset \Omega, j=1, \ldots, N^{\prime}$ be a set of mutually neighborhoods such that $\partial \Omega \subset \cup_{j=1}^{N}\left(U_{j} \cup V_{j}\right)$. Set $W:=\bigcup_{j=1}^{N} U_{j} \cup \bigcup_{j=1}^{N^{\prime}} V_{j} \subset \Omega$. $W$ is obviously a neighborhood of $\partial \Omega$ in $\Omega$. Its complement is a unbounded open set which is bounded away from $\partial \Omega$. Let $\theta_{j}, j=1, \ldots, N$ and $\Phi_{j}, j=1, \ldots, N$ be cut-off functions supported in $U_{j}$ and $V_{j}$, respectively. We demand that $\Phi_{j} \equiv 1$ in a neighborhood of $v_{j}$, and $\sum_{j=1}^{N}\left(\Phi_{j}+\Theta_{j}\right) \equiv 1$ in a neighborhood of $\partial \Omega$. We call a the family of pairs $\left\{\left(\Phi_{j}, \Theta_{j}\right) \mid j=1, \ldots, N\right\}$ a partition of unity adapted to the boundary $\partial \Omega$.

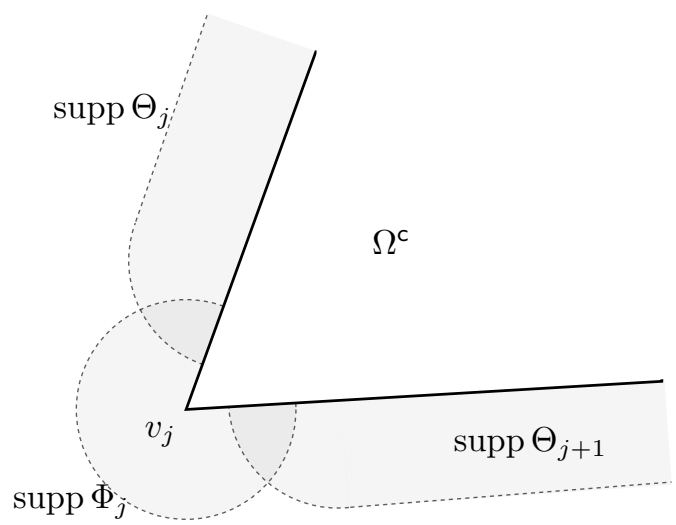

Figure 4.1: The cut-off functions $\Phi_{j}, \Theta_{j}$, and $\Theta_{j+1}$.

Note that this definition, while not necessary for the following definitions, will be helpful for our calculations in Chapter 6. In the following sections, we will make frequent use of pairs of cut-off functions $\Theta_{j}^{\prime} \Theta_{j}=\Theta_{j}$ and $\Phi_{j}^{\prime} \Phi_{j}=\Phi_{j}$ as they are defined above.

\subsection{Interior Operators}

Definition 4.2. Denote by $M_{P}^{\mu}(X)$ the Fréchet space consiting of all operator families $h(z) \in \mathcal{A}\left(\mathbb{C}, L_{\mathrm{cl}}^{\mu}(X)\right)$ such that $h(\beta+i \rho) \in L_{\mathrm{cl}}^{\mu}(X)$ for all $c \leq \beta \leq c^{\prime}$, $c<c^{\prime}$.

Let $h \in C^{\infty}\left(\overline{\mathbb{R}}_{+} \times \overline{\mathbb{R}}_{+}, M_{P}^{\mu}(X)\right)$ for $\mu \in \mathbb{R}$ and a Mellin asymptotic type $P$. 
Then, for $f \in \mathcal{H}^{s, \gamma}\left(X^{\wedge}\right)$, we define the operator op ${ }_{M}^{\gamma}[h] f$ by

$$
\mathrm{op}_{\mathrm{M}}^{\gamma}[h] f:=\frac{1}{2 \pi} \int_{\Gamma_{\frac{n+1}{2}-\gamma}} \int_{0}^{\infty}\left(\frac{r}{r^{\prime}}\right)^{-z} h\left(r, r^{\prime}, z\right) f(r) \frac{d r^{\prime}}{r^{\prime}} d z .
$$

One can show that $\operatorname{op}_{\mathrm{M}}^{\gamma}[h] f \in \mathcal{H}^{s-\mu, \gamma}\left(X^{\wedge}\right)$. As we will observe during the course of our work, the Mellin symbols also depend holomorphically on a complex parameter $\lambda \in U_{\lambda}, U_{\lambda} \subset \mathbb{C}$ a domain. Therefore, we introduce the spaces

$$
\mathcal{A}\left(U_{\lambda}\right) \hat{\otimes}_{\pi} M_{P}^{\mu}(X) \cong \mathcal{A}\left(U_{\lambda}, M_{P}^{\mu}(X)\right)
$$

of Mellin symbol-valued holomorphic functions.

Definition 4.3. A parameter-dependent interior operator A of order $\mu$ is a family of operators $\{\mathrm{A}(\lambda) \mid \lambda \in U\}$ of the form

$$
\mathrm{A}(\lambda)=\mathrm{A}_{\mathrm{M}}(\lambda)+\mathrm{A}_{\psi}(\lambda)+A_{\mathrm{M}}(\lambda)
$$

where

1. $\mathrm{A}_{\mathrm{M}}=\sum_{j=1}^{N} \Phi_{j}^{\prime} \mathrm{op}_{\mathrm{M}}^{\gamma}\left[h_{j}\right] \Phi_{j}, h_{j} \in \mathcal{A}\left(\mathbb{C}, M^{\mu}\left(X_{j}\right)\right)$ for a $(n-1)$ dimensional base manifold $X_{j}$,

2. $\mathrm{A}_{\psi}=\sum_{j=1}^{N} \Theta_{j}^{\prime} \mathrm{op}_{\psi}\left[h_{j}^{\psi}\right] \Theta_{j}, h_{j}^{\psi} \in S^{\mu}(I \times I \times \mathbb{R})$.

3. $A_{M}$ is a smoothing operator.

The algebra of interior operators depending on a complex parameter will be denoted by $\mathcal{I}^{\mu}(\Omega ; U)$.

\subsection{Potential Operators}

One peculiarity of the theory for two dimensional singular domains is that the conical boundary can be decomposed into two connected components. In other words, $\partial X^{\wedge}=Y_{1} \cup Y_{2}$ for a sector $X^{\wedge} \subset \mathbb{R}^{2}$, where $Y_{i} \cong \mathbb{R}_{+}$for $i=1,2$. Let $\mu \in \mathbb{R}$ and $P$ a Mellin asymptotic type. For

$$
\bar{h}:=\left(h_{1}, h_{2}\right) \in M_{P}^{\mu} \oplus M_{P}^{\mu}
$$

we denote the corresponding operator by

$$
\mathrm{op}_{\mathrm{M}}^{\gamma}[\bar{h}]\left[\begin{array}{l}
f_{1} \\
f_{2}
\end{array}\right]:=\mathrm{op}_{\mathrm{M}}^{\gamma}\left[h_{1}\right] f_{1}+\mathrm{op}_{\mathrm{M}}^{\gamma}\left[h_{2}\right] f_{2} \in \mathcal{H}^{s+\frac{1}{2}-\mu, \gamma+\frac{1}{2}}\left(X^{\wedge}\right),
$$

where $f_{i} \in \mathcal{H}^{s, \gamma}\left(Y_{i}\right), i=1,2$. Note that we have

$$
\mathcal{A}\left(U_{\lambda}, M_{P}^{\mu} \oplus M_{P}^{\mu}\right) \cong \mathcal{A}\left(U_{\lambda}, M_{P}^{\mu}\right) \oplus \mathcal{A}\left(U_{\lambda}, M_{P}^{\mu}\right) .
$$

Of course, we could have chosen different orders and asymptotic types for the two components $h_{1}$ and $h_{2}$ of the symbol (4.1). But since we do not need this freedom, we choose both to be equal.

In order to define potential operators on the whole boundary $\partial \Omega$, we need to index the symbols by a subscript. Mind however, that the boundary in a 
neighborhood of the vertex $v_{j}$ splits up into two components, namely $\partial \Omega_{j}$ and $\partial \Omega_{j+1}$. With $\left.\Phi_{j}\right|_{\partial \Omega_{j}}=\phi_{j}^{j}$ and $\left.\Phi_{j}\right|_{\partial \Omega_{j+1}}=\phi_{j}^{j+1}$, we can further define the 'matrix-valued' cut-off functions

$$
\hat{\phi}_{j}:=\left.\Phi_{j}\right|_{\partial \Omega}=\left[\begin{array}{cc}
\phi_{j}^{j+1} & 0 \\
0 & \phi_{j}^{j}
\end{array}\right] .
$$

These 'cut-off matrices' map $\mathcal{H}^{s, \gamma}\left(\partial \Omega_{j}\right) \oplus \mathcal{H}^{s, \gamma}\left(\partial \Omega_{j+1}\right)$ onto itself.

Definition 4.4. Let $U \subseteq \mathbb{C}$ be a domain. A parameter-dependent potential operator K of order $\mu$ on $\Omega$ is a family $\{\mathrm{K}(\lambda) \mid \lambda \in U\}$ of operators $\mathrm{K}(\lambda)$ : $C_{0}^{\infty}(\partial \Omega) \rightarrow \mathcal{D}^{\prime}(\Omega)$ of the form

$$
\mathrm{K}(\lambda)=\mathrm{K}_{\mathrm{M}}(\lambda)+\mathrm{K}_{\psi}(\lambda)+\mathrm{K}_{\mathrm{G}}(\lambda) .
$$

We require these operators, when localized, to have the following form:

1. $\mathrm{K}_{\mathrm{M}}(\lambda)=\sum_{j=1}^{N} \Phi_{j}^{\prime} \mathrm{K}(\lambda) \hat{\phi}_{j}$, where $\Phi_{j} \mathrm{~K}_{\mathrm{M}}(\lambda) \hat{\phi}_{j}=\Phi_{j} \mathrm{op}_{\mathrm{M}}\left[\bar{h}_{j}(\lambda)\right] \hat{\phi}_{j}$ with Mellin symbols $\bar{h}_{j} \in \mathcal{A}\left(U, M^{\mu}\right) \oplus \mathcal{A}\left(U, M^{\mu}\right)$.

2. $\mathrm{K}_{\psi}(\lambda)=\sum_{j=1}^{N} \theta_{j}^{\prime} \mathrm{K}(\lambda) \theta_{j}$, where $\mathrm{K}_{\psi}(\lambda)$ are ordinary (Fourier) pseudodifferential operators with symbols $h^{\psi} \in \mathcal{A}(U) \hat{\otimes}_{\pi} S^{\mu}\left(I \times I, \mathbb{R} ; \mathcal{S}\left(\mathbb{R}_{+}\right)\right), I \subset$ $\partial \Omega_{j}$ an open interval.

3. Finally, $\mathrm{K}_{\mathrm{G}}$ is a regulating operator, that is $\mathrm{K}_{\mathrm{G}}(\lambda): \mathcal{H}^{s, \gamma}(\partial \Omega) \rightarrow C^{\infty}(\bar{\Omega})$. It has locally the form $\mathrm{K}_{\mathrm{G}}(\lambda)=\sum_{j=1}^{N} \Phi_{j}^{\prime} \mathrm{K}(\lambda)\left(1-\hat{\phi}_{j}\right)$.

The set of potential operators with holomorphic dependency in $U$ will be denoted by $\mathcal{P}^{\mu}(\Omega ; U)$.

As we will see in a moment, the potential operators are the operators in the upper right corner of the elements of Boutet de Monvel's calculus.

\subsection{The Operators on the Boundary}

Definition 4.5. We define the Mellin symbol at the vertex $v_{j}$ by the matrix

$$
\hat{h}(\lambda):=\left[\begin{array}{ll}
h^{1,1}(\lambda) & h^{1,2}(\lambda) \\
h^{2,1}(\lambda) & h^{2,2}(\lambda)
\end{array}\right],
$$

with $h^{1,1}(\lambda), h^{2,2}(\lambda) \in M_{P}^{\mu}$, and the off-diagonal elements $h^{1,2}(\lambda)$ and $h^{2,1}(\lambda)$ in $M_{P}^{-\infty}$ for some Mellin asymptotic type $P$. We denote by op $\mathrm{M}_{\mathrm{M}}^{\gamma}[\hat{h}(\lambda)]$ the operator

$$
\left[\begin{array}{ll}
\operatorname{op}_{M}^{\gamma}\left[h^{1,1}(\lambda)\right] & \operatorname{op}_{M}^{\gamma}\left[h^{1,1}(\lambda)\right] \\
\operatorname{op}_{M}^{\gamma}\left[h^{2,1}(\lambda)\right] & \operatorname{op}_{M}^{\gamma}\left[h^{2,2}(\lambda)\right]
\end{array}\right]\left[\begin{array}{l}
f_{1} \\
f_{2}
\end{array}\right] \in \mathcal{H}^{s, \gamma}\left(Y_{1}\right) \oplus \mathcal{H}^{s, \gamma}\left(Y_{2}\right),
$$

for $f_{i} \in \mathcal{H}^{s, \gamma}\left(Y_{i}\right), i=1,2$. We denote this space of Mellin symbols by $M_{P}^{\mu}\left(\mathbb{M}_{2 \times 2}\right)$.

An easy consequence of this definition is (cf. Lemma 3.24):

Lemma 4.6. For $\hat{h}_{1}$ and $\hat{h}_{2} \in M_{P}^{\mu}\left(\mathbb{M}_{2 \times 2}\right)$ we have

$$
\operatorname{op}_{\mathrm{M}}^{\gamma}\left[\hat{h}_{1} \hat{h}_{2}\right]=\mathrm{op}_{\mathrm{M}}^{\gamma}\left[\hat{h}_{1}\right] \operatorname{op}_{\mathrm{M}}^{\gamma}\left[\hat{h}_{2}\right] \text {, }
$$

with $\hat{h}_{1} \hat{h}_{2}$ being the $2 \times 2$-matrix product of $\hat{h}_{1}$ and $\hat{h}_{2}$. 
Having discussed this model case for a corner, we can proceed to define the potential operators for the whole boundary. As in the case of the Mellin operators on the boundary, we will introduce subscripts to our symbols.

Definition 4.7. Let $h_{j}, j=1, \ldots, N$, be a family of Mellin symbols of the form (4.3). We define the algebra $\mathcal{C}^{\mu}(\partial \Omega, \mathbf{g} ; U)$ as the set of operators with meromorphic dependence on the parameter $\lambda \in U$ as the family of operators $A(\lambda)$ with the following decomposition:

$$
\mathrm{A}(\lambda)=\mathrm{A}_{\mathrm{M}}(\lambda)+\mathrm{A}_{\psi}(\lambda)+\mathrm{A}_{\mathrm{G}}(\lambda),
$$

where

1. $\mathrm{A}_{\mathrm{M}}(\lambda)=\sum_{j=1}^{N} \hat{\phi}_{j}^{\prime} \mathrm{op}_{\mathrm{M}}^{\gamma}\left[\hat{h}_{j}(\lambda)\right] \hat{\phi}_{j}$,

2. $\mathrm{A}_{\psi}(\lambda)=\sum_{j=1}^{N} \theta_{j}^{\prime} \mathrm{op}_{\psi}\left[h_{j}^{\psi}(\lambda)\right] \theta_{j}$,

3. $\mathrm{A}_{\mathrm{G}}(\lambda)=\sum_{j=1}^{N} \mathrm{G}_{j}(\lambda)$

with $\hat{h}_{j} \in M_{P}^{\mu}\left(\mathbb{M}_{2 \times 2}\right), h_{j}^{\psi} \in \mathcal{A}(U) \hat{\otimes}_{\pi} S^{\mu}(I \times I \times \mathbb{R})$. and $\mathrm{G}_{j} \in \mathcal{C}_{\mathrm{G}}(\partial \Omega, \mathbf{g} ; U)$ are Green operators.

We are in a position to define the principal symbol of an operator in the calculus on $\partial \Omega$. In contrast to the calculus on the real half line $\mathbb{R}_{+}$, we will have to deal with a whole family of symbols, parametrized by the set of vertices $\left\{v_{1}, \ldots, v_{N}\right\}$.

Definition 4.8. The family

$$
\sigma^{m}(\mathrm{~A}(\lambda))=\left(\sigma_{\mathrm{M}, \mathrm{j}}(\mathrm{A}(\lambda)), \sigma_{\psi, \mathrm{j}}(\mathrm{A}(\lambda))\right)_{j=1, \ldots, N}
$$

of pairs, where

$$
\sigma_{\mathrm{M}, \mathrm{j}}(\mathrm{A}(\lambda)):=\left[\begin{array}{ll}
h_{j}^{1,1}(z) & h_{j}^{1,2}(z) \\
h_{j}^{2,1}(z) & h_{j}^{2,2}(z)
\end{array}\right]
$$

and

$$
\sigma_{\psi, \mathrm{j}}(\mathrm{A}(\lambda)):=\sigma_{\psi}\left(h_{j}^{\psi}(\lambda)\right)
$$

is called the principal symbol of the operator $\mathrm{A} \in \mathcal{C}^{m}(\partial \Omega, \mathbf{g} ; U)$. Here, $\sigma^{\psi}\left(h_{j}^{\psi}\right)$ denotes the principle part of the symbol.

Definition 4.9. We define the operator class $L^{\infty}(\partial \Omega)$ as the smoothing operator in the interior, $\mathcal{C}_{\mathrm{M}+\mathrm{G}}(X, h)$ as the smoothing operator at the corner.

Theorem 4.10 (Multiplication of operators). Let $\mathrm{A} \in \mathcal{C}^{m}(\partial \Omega, \mathbf{g})$, $\tilde{\mathrm{A}} \in \mathcal{C}^{\tilde{m}}(\partial \Omega, \tilde{\mathbf{g}})$ with $(\gamma, \gamma-m, \Theta)$ and $\tilde{\mathbf{g}}=(\gamma-m, \gamma-(m+\tilde{m}), \Theta)$. Then $\mathrm{A} \tilde{\mathrm{A}} \in \mathcal{C}^{m+\tilde{m}}(\partial \Omega, \mathbf{h}), \mathbf{h}=(\gamma, \gamma-(m+\tilde{m}), \Theta)$, with the principal conormal symbol given by the Mellin translation product:

$$
\sigma_{\mathrm{M}, \mathrm{j}}^{\mu+\tilde{\mu}}(\mathrm{A} \tilde{\mathrm{A}})(z)=\left\{\tau^{\tilde{\mu}} \sigma_{\mathrm{M}, \mathrm{j}}^{\mu}(\mathrm{A})(z)\right\} \sigma_{\mathrm{M}, \mathrm{j}}^{\tilde{\mu}}(\tilde{\mathrm{A}})(z) .
$$

The interior principal symbol of the product $\mathrm{A} \tilde{\mathrm{A}}$ is simply given by

$$
\sigma_{\psi}^{\mu+\tilde{\mu}}(\mathrm{A} \tilde{\mathrm{A}})=\sigma_{\psi}^{\tilde{\mu}}(\mathrm{A}) \sigma_{\psi}^{\mu}(\tilde{\mathrm{A}}) .
$$


The composition of Mellin pseudodifferential operators plays a central role in this thesis, since an important part is devoted to the construction of a parametrix for the boundary value of the single layer potential. Instead of proving the theorem here, we give a more detailed account when actually we need the composition in the proof of theorem 7.1.

Lemma 4.11. Let $\mathrm{A} \in \mathcal{C}^{\mu}(\partial \Omega, \mathbf{g})$ and $\mathrm{B} \in \mathcal{C}^{\tilde{\mu}}(\partial \Omega, \tilde{\mathbf{g}})$ smoothing. Then $\mathrm{AB}$ is a smoothing Mellin operator of conormal order $\mu+\tilde{\mu}$.

Lemma 4.12. A product of a smoothing Mellin and a Green operator is a Green operator, and if $\mathrm{A} \in \mathcal{C}^{\mu}(\partial \Omega, \mathbf{g}), \mathrm{B} \in \mathcal{C}^{\tilde{\mu}}(\partial \Omega, \tilde{\mathbf{g}})$ are both smoothing with Mellin symbols $h_{1}$ and $h_{2}$, then the product $\mathrm{AB} \in \mathcal{C}^{\mu+\tilde{\mu}}(\partial \Omega, \mathbf{h}), \mathbf{h}=(\gamma, \gamma-(m+\tilde{m}), \Theta)$ is a sum of an Mellin operator with a Mellin symbol $h$ and a Green operator.

Remark 4.13. The conormal symbols of $\mathrm{AB}$ can be calculated by the Mellin translation product, i.e.

$$
\sigma_{\mathrm{M}}^{\mu+\tilde{\mu}-j}(\mathrm{AB})(z)=\sum_{p+q=j}\left(\tau^{\mu-j} \sigma_{\mathrm{M}}^{\tilde{\mu}-j}(\mathrm{~A})\right)(z) \sigma_{\mathrm{M}}^{m-q}(\mathrm{~B})(z)
$$

for $j=0,1, \ldots, k-1$.

The notion of ellipticity for the calculus $\mathcal{C}^{\mu}(\partial \Omega, \mathbf{g}), \mu \in \mathbb{R}$, is a slight modification of Definition 3.32. In theorem 7.1 we are going to proof that the potential operator in our scattering theory is in fact elliptic.

Definition 4.14 (Ellipticity). An operator in the calculus $\mathcal{C}^{\mu}(\partial \Omega, \mathbf{g})$ will be called elliptic, if the internal symbols are elliptic in the sense of ordinary Pseudodifferential operators, and all the principal conormal symbols associated with the vertices are invertible matrices on the line $\Gamma_{\frac{1}{2}-\gamma}$.

This enables us, according to the general theory of Mellin pseudodifferential operators to construct a parametrix:

Theorem 4.15 (Fredholm property and Parametrix). If A is elliptic of order $\mu$ and conormal order $m$, then is Fredholm for all $s \in \mathbb{R}$. There exists a parametrix $\mathrm{Q}$ of $A$ in the sense of. Furthermore,

$$
\mathrm{A} u=f \in \mathcal{H}^{s-m, \gamma-m}(\partial \Omega)
$$

for any $s \in \mathbb{R}$ and

$$
u \in \mathcal{H}^{-\infty, \gamma}(\partial \Omega)
$$

implies $u \in \mathcal{H}^{s, \gamma}(\partial \Omega)$. Finally,

$$
\mathrm{A} u=f \in \mathcal{H}_{P_{1}}^{s, \gamma}(\partial \Omega)
$$

for some asymptotic type $P_{1}$ to $(\gamma-m, \Theta)$ and $u \in \mathcal{H}^{-\infty, \gamma}(\partial \Omega)$ imply $\mathcal{H}_{P_{2}}^{s, \gamma}$ with an asymptotic type $P_{2}$ to $(\gamma, \Theta)$ for all $s \in \mathbb{R}$.

The proof of this theorem is exactly the same as in the case of calculi on conic spaces $X^{\wedge}$. 


\subsection{Boutet de Monvel's Algebra}

Since we only need the subalgebra of operators on the boundary, we will solely outline the definition without going into any details.

$$
\left[\begin{array}{cc}
\Phi_{j} & 0 \\
0 & \left.\Phi_{j}\right|_{\partial \Omega}
\end{array}\right]
$$

Definition 4.16. Let $\Omega \subset \mathbb{R}^{n}$ be an exterior domain with polygonal boundary $\partial \Omega$. Let $U \subset \mathbb{C}$ be an open subset. The idea of Boutet de Monvel is to formulate a calculus of operators with elements $\mathcal{A} \in \mathcal{B}^{\mu}(\Omega ; U):=\mathcal{A}\left(U, \mathcal{B}^{\mu}(\Omega)\right)$ of the form, $\lambda \in U$,

$$
\mathcal{A}(\lambda)=\left[\begin{array}{ll}
\mathrm{A}(\lambda) & \mathrm{K}(\lambda) \\
\mathrm{T}(\lambda) & \mathrm{Q}(\lambda)
\end{array}\right]: \begin{array}{cccc}
C^{\infty}(\bar{\Omega}) & & C^{\infty}(\bar{\Omega}) \\
& C^{\infty}(\partial \Omega) & & C^{\infty}(\partial \Omega)
\end{array}
$$

which is a non-pseudodifferential term which has to be included to make compositions in the algebra work. Notice that this operator is the one which inspired the naming of the Green operator in singular analysis.

The elements of the algebra are localized in such a way that they are either supported in a neighborhood $U_{j}$ of a corner $v_{j}$ or in a neighborhood $V_{j}$ of the smooth interior of an edge $\partial \Omega_{j}$. Along the smooth parts of the boundary, the algebra is constructed in a straightforward manner (cf. [Gru09] or [SS94] for details), whereas in the vicinity of the vertices, meromorphic Mellin symbols with values in the Boutet de Monvel algebra of the base space of the corresponding cone come into play [SS95]. Defining the algebra in the exterior domain is then done as follows:

Definition 4.17. We write $A \in \mathcal{B}^{\mu}(\Omega ; U)$ for operators

$$
\mathcal{A}(\lambda): \begin{gathered}
C^{\infty}(\bar{\Omega}) \\
C^{\infty}(\partial \Omega)
\end{gathered} \rightarrow \begin{gathered}
C^{\infty}(\bar{\Omega}) \\
C^{\infty}(\partial \Omega)
\end{gathered} .
$$

depending on a complex parameter $\lambda \in \mathcal{U}$ which can be written in the form

$$
A=\sum_{j=1}^{N} \hat{\Phi}_{j} \mathrm{~A} \hat{\Psi}_{j}+\sum_{j=1}^{N} \hat{\Phi}_{j} A\left(1-\hat{\Psi}_{j}\right)
$$

and satisfy the following conditions

1. For every $j$, the operator $\hat{\Phi}_{j} A \hat{\Psi}_{j}$ belongs to $\mathcal{B}^{\mu}\left(V_{j} ; U\right)$.

2. For every $j$, the operator $\hat{\Phi}_{j} A \hat{\Psi}_{j}$ belongs to $\mathcal{B}^{\mu}\left(U_{j} ; U\right)$.

3. The sum $\sum_{j=1}^{N} \hat{\Phi}_{j} A\left(1-\hat{\Psi}_{j}\right)$ is induced by an integral operator

$$
C^{\infty}(\bar{\Omega}) \oplus C^{\infty}(\partial \Omega) \rightarrow C^{\infty}(\bar{\Omega}) \oplus C^{\infty}(\partial \Omega)
$$

depending on the complex parameter $\lambda \in U$. This integral operator has a kernel of $C^{\infty}$-class.

We denote the following subsets of the algebra $\mathcal{B}^{\mu, d}(\Omega ; U)$ as follows: 


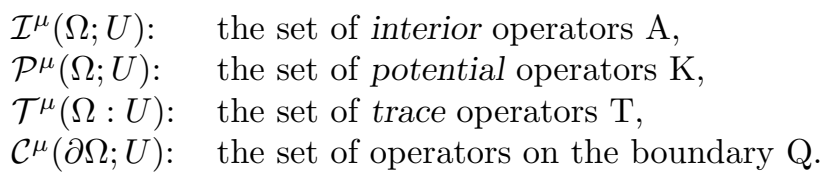

Once again, $\mathcal{C}^{\mu, d}(\partial \Omega)$ is an algebra in its own right. It is this algebra in which the traces of the layer potentials belong to, and in which we will construct the parametrix Q.

\subsection{Calderón Projectors}

Definition 4.18. The Calderón projectors associated with the potential operator $K(\lambda)$ are definied as the traces of the potential operators, i.e.

$$
\mathrm{C}^{ \pm}(\lambda):=\gamma^{ \pm} \mathrm{K}^{ \pm}(\lambda) .
$$

The second row of the Calderón projector, denoted as

$$
\mathrm{N}^{ \pm}(\lambda)=\gamma_{1}^{ \pm} \mathrm{K}^{ \pm}(\lambda),
$$

is called the Dirichlet-to-Neumann-operator.

Depending on whether one takes the trace on the exterior $\left({ }^{\prime}+^{\prime}\right)$ or the interior $\left({ }^{\prime}-^{\prime}\right)$ side of the domain's boundary. The Calderón projector for the exterior domain, $\mathrm{C}^{+}(\lambda)$, written as a $2 \times 2$-Matrix, is given by

$$
\mathrm{C}^{+}(\lambda)=\left[\begin{array}{ll}
\mathrm{C}_{00}^{+}(\lambda) & \mathrm{C}_{01}^{+}(\lambda) \\
\mathrm{C}_{10}^{+}(\lambda) & \mathrm{C}_{11}^{+}(\lambda)
\end{array}\right]
$$

The Calderón projector is indeed a projection, i.e. it satisfies $\left(\mathrm{C}^{+}\right)^{2}=\mathrm{C}^{+}$. The Calderón projector for the exterior and interior domains are related by $\mathrm{C}^{-}(\lambda)=\left(\mathbb{1}-\mathrm{C}^{+}(\lambda)\right)$. They are (Mellin) pseudodifferential operators on the boundary of the domain, which means that with regards to Boutet de Monvel's, they are of the form

$$
\mathcal{A}_{\text {Calderón }}^{ \pm}=\left[\begin{array}{cc}
0 & 0 \\
0 & \mathrm{C}^{ \pm}(\lambda)
\end{array}\right] .
$$

For the scattering problem, one usually only considers the outer side of the boundary. Therefore, we shall omit the plus sign when referencing to the exterior domain, i.e. $\mathrm{C}_{01}(\lambda)=\mathrm{C}_{01}^{+}(\lambda)$. 


\section{Chapter 5}

\section{Basic Results}

\subsection{Pseudodifferential Boundary Problems}

Consider the scattering problem with Dirichlet boundary conditions:

$$
\left\{\begin{aligned}
\left(\Delta+\lambda^{2}\right) u_{\lambda}^{\mathrm{i} / \mathrm{o}} & =f \quad \text { in } \Omega, \\
\gamma_{0}^{+} u_{\lambda}^{\mathrm{i} / \mathrm{o}} & =g \\
\lim _{r \rightarrow \infty} r^{\frac{1}{2}}\left(\partial_{r} \mp i \lambda\right) u_{\lambda}^{\mathrm{i} / \mathrm{o}} & =0 .
\end{aligned}\right.
$$

Here, $\gamma_{0}: H^{s}(\Omega) \rightarrow H^{s-\frac{1}{2}}(\partial \Omega), s>1 / 2$, is the conventional trace operator for Sobolev spaces on smooth hypersurfaces, and $\operatorname{rad}_{\lambda}^{\mathrm{i} / \mathrm{o}}$ the operator implementing the outgoing ('rad+') or incoming ('rad_') radiation conditions, respectively:

$$
\operatorname{rad}_{\lambda}^{\mathrm{i} / \mathrm{o}} u(\omega):=\lim _{r \rightarrow \infty} r^{\frac{1}{2}}\left(\partial_{r} u(r \omega) \mp i \lambda u(r \omega)\right), \quad r>0, \omega \in \mathbb{S}^{1} .
$$

For the scattering problem (5.1), we may set

$$
\mathrm{P}(\lambda):=\Delta+\lambda^{2}: C^{\infty}(\Omega) \longrightarrow C^{\infty}(\Omega),
$$

whereas the trace operator is given by the column matrix

$$
\mathrm{T}^{\mathrm{i} / \mathrm{o}}(\lambda):=\left[\begin{array}{c}
\gamma_{0}^{+} \\
\operatorname{rad}_{\lambda}^{\mathrm{i} / \mathrm{o}}
\end{array}\right]: C^{\infty}(\dot{\Omega}) \rightarrow \begin{gathered}
C^{\infty}(\partial \Omega) \\
C^{\infty}\left(\mathbb{S}^{1}\right)
\end{gathered} .
$$

In the well-known (cf. [Tay13]) that the general solution of this boundary value problem may be written as

$$
u_{\lambda}(x)=\mathrm{R}^{\mathrm{i} / \mathrm{o}}(\lambda) f(x)+\mathrm{K}^{\mathrm{i} / \mathrm{o}}(\lambda) g(x), \quad x \in \Omega \cup \Omega^{\mathrm{c}}, \lambda \in \Lambda,
$$

where $\mathrm{R}^{\mathrm{i} / \mathrm{o}}$ is the resolvent of the problem with homogeneous Dirichlet boundary conditions, and $\mathrm{K}^{\mathrm{i} / \mathrm{o}}$ is a linear combination of the single and double layer surface potentials.

Definition 5.1. Let $s, \gamma \in \mathbb{R}, P$ an asymptotic type. Then we define the space

$$
\mathcal{K}_{\mathrm{i} / \mathrm{o}}^{s, \gamma}(\Omega):=\left\{u \in \mathcal{K}^{s, \gamma}(\Omega) \mid \operatorname{rad}_{\lambda}^{\mathrm{i} / \mathrm{o}} u=0\right\}
$$


We are initially defining them as

$$
\mathrm{R}^{\mathrm{i} / \mathrm{o}}(\lambda): C_{0}^{\infty}(\Omega) \rightarrow C^{\infty}(\Omega),
$$

fulfilling the conditions

$$
\left\{\begin{aligned}
\left(\Delta+\lambda^{2}\right) \mathrm{R}^{\mathrm{i} / \mathrm{o}}(\lambda) & =\mathbb{1} \quad \text { in } \Omega, \\
\gamma_{0}^{+} \mathrm{R}^{\mathrm{i} / \mathrm{o}}(\lambda) & =0 \\
\operatorname{rad}_{\lambda}^{\mathrm{i} / \mathrm{o}} \mathrm{R}^{\mathrm{i} / \mathrm{o}}(\lambda) & =0
\end{aligned}\right.
$$

and as the operator

$$
\mathrm{K}^{\mathrm{i} / \mathrm{o}}(\lambda): C^{\infty}(\partial \Omega) \rightarrow C^{\infty}(\bar{\Omega}),
$$

with the properties

$$
\left\{\begin{aligned}
\left(\Delta+\lambda^{2}\right) \mathrm{K}^{\mathrm{i} / \mathrm{o}}(\lambda) & =0 \quad \text { in } \Omega, \\
\gamma_{0}^{+} \mathrm{K}^{\mathrm{i} / \mathrm{o}}(\lambda) & =\mathbb{1}, \\
\operatorname{rad}_{\lambda}^{\mathrm{i} / \mathrm{o}} \mathrm{K}^{\mathrm{i} / \mathrm{o}}(\lambda) & =0 .
\end{aligned}\right.
$$

We will demonstrate the existence of these solution operators and their continuation to more general function spaces. This will be the content of chapter 6 . Instead of proceeding as in [Tay13], we will introduce the unifying beauty of pseudodifferential boundary operators and Boutet de Monvel's algebra. The solution operator may then be written as

$$
\mathcal{A}^{-1}(\lambda):=\left[\begin{array}{cc}
\mathrm{R}^{\mathrm{i} / \mathrm{o}}(\lambda) & \mathrm{K}^{\mathrm{i} / \mathrm{o}}(\lambda) \\
0 & 0
\end{array}\right] .
$$

The solution is then given by

$$
u(x):=\mathrm{R}^{\mathrm{i} / \mathrm{o}}(\lambda) f+\mathrm{K}^{\mathrm{i} / \mathrm{o}}(\lambda) g=\pi_{1}\left(\mathcal{A}^{-1}(\lambda)\left[\begin{array}{l}
f \\
g
\end{array}\right]\right),
$$

where the square brackets indicate the projection onto the first component of a vector.

Although Boutet de Monvel's framework offers conceptual clarity, the hard work of a mathematical analyst still remains: To show that the problem (5.1) is wellposed, i.e. both operators exist and are continuous. The interior operator will be dealt with in the following section. More involved however is the potential operator, where a thorough treatment can only be done with the full machinery of Mellin pseudodifferential operators. This will be the main topic of chapter 6 .

\subsection{The Free Resolvent}

The fundamental solution for the Helmholtz equation is of major importance in the study of scattering poles. Its asymptotic expansion will be important when discussing its mapping properties and parametrix construction in chapters 6 and 7. The fundamental solutions for the free problem in two spatial dimensions are given, depending on the radiation condition, by either a Hankel function of the first kind, or the second. ${ }^{1}$ Denote the Hankel functions of first and second kinds and order zero by $H_{0}^{(i)}, i=1,2$, respectively.

\footnotetext{
${ }^{1}$ For general information on special functions see for example [Wat66] or [Olv74].
} 
Definition 5.2. Let $x \neq y$ and $\lambda \in \Lambda$, where $\Lambda$ denotes the Riemann surface of the logarithm. The outgoing fundamental solution is then given by

$$
r_{0}^{\text {out }}(\lambda|x-y|):=-\frac{i}{4}\left(\frac{\lambda}{2 \pi|x-y|}\right)^{\frac{n-2}{2}} H_{0}^{(2)}(\lambda|x-y|)
$$

or by

$$
r_{0}^{\text {in }}(\lambda|x-y|):=\frac{i}{4}\left(\frac{\lambda}{2 \pi|x-y|}\right)^{\frac{n-2}{2}} H_{0}^{(1)}(\lambda|x-y|),
$$

for the incoming Sommerfeld radiation condition.

As we will see later (equation 6.8), the fundamental solutions are both related to each other by

$$
r_{0}^{\text {out }}(\lambda \tau)=r_{0}^{\text {in }}(\lambda \tau)-\frac{i}{2}\left(\frac{\lambda}{2 \pi \tau}\right)^{\frac{n-2}{2}} J_{\frac{n-2}{2}}(\lambda \tau), \quad \tau>0,
$$

where $J_{\frac{n-2}{2}}$ is the Bessel function. Note that the Bessel function may be expanded as, $\nu \in \mathbb{R}$,

$$
J_{\nu}(z)=\left(\frac{1}{2} z\right)^{\nu} \sum_{s=0}^{\infty}(-1)^{s} \frac{\left(\frac{1}{4} z^{2}\right)^{s}}{s ! \cdot \Gamma(\nu+s+1)} .
$$

For $\nu=0$, this expansion takes the form

$$
J_{0}(\lambda \tau)=\sum_{k=0}^{\infty} \frac{(-1)^{k}}{(k !)^{2}}\left(\frac{\lambda}{2}\right)^{2 k} \tau^{2 k}
$$

One major aspect of the fundamental solutions are their limits at infinity.

Lemma 5.3. Let $y \in \mathbb{R}^{2}$ and $\lambda \in \Lambda$ be fixed. Then

$$
r_{0}^{\mathrm{i} / \mathrm{o}}(\lambda|x-y|)=\frac{1}{2 \sqrt{\lambda}} \frac{1}{\sqrt{2 \pi}} \frac{e^{\mp i \lambda|x|}}{\sqrt{|x|}} e^{\mp i \lambda \mid \frac{x}{|x|} y}+O\left(\frac{1}{|x|}\right)
$$

and

$$
\frac{\partial r_{0}^{\mathrm{i} / \mathrm{o}}}{\partial \nu(x)}(\lambda|x-y|)=\frac{1}{2 \sqrt{\lambda}} \frac{1}{\sqrt{2 \pi}} \frac{e^{\mp i \lambda|x|}}{\sqrt{|x|}} e^{\mp i \lambda \frac{x}{|x|} y}+O\left(\frac{1}{|x|}\right)
$$

as $|x| \rightarrow \infty$.

Proof. We have for the Hankel funktions for $|z| \rightarrow \infty$ the asymptoticals [Olv74]:

$$
\begin{aligned}
& H_{0}^{(1)}(z) \sim \sqrt{\frac{2}{\pi z}} e^{i\left(z-\frac{\pi}{4}\right)} \sum_{s=0}^{\infty} i^{s} \frac{A_{s}(0)}{z^{s}} \quad \text { for }-\pi<\arg (z)<2 \pi, \\
& H_{0}^{(2)}(z) \sim \sqrt{\frac{2}{\pi z}} e^{-i\left(z-\frac{\pi}{4}\right)} \sum_{s=0}^{\infty}(-i)^{s} \frac{A_{s}(0)}{z^{s}} \quad \text { for }-2 \pi<\arg (z)<\pi,
\end{aligned}
$$

where

$$
A_{s}(0)=(-1)^{s} \frac{\left(1^{2}\right)\left(3^{2}\right) \cdots(2 s-1)^{2}}{s ! \cdot 8^{s}}
$$


In addition we have for $|x| \rightarrow \infty$ :

$$
|x-y|=|x| \sqrt{1-2 \frac{x y}{|x|^{2}}+y^{2}}=|x|-\frac{x}{|x|} y+O\left(\frac{1}{|x|}\right) .
$$

Now set $\omega:=x /|x| \in \mathbb{S}^{1}$. Then:

$$
r_{0}^{\mathrm{i} / \mathrm{o}}(\lambda|x-y|)=\frac{1}{2 \sqrt{\pi}} \frac{1}{\sqrt{2 \pi}} \frac{e^{\mp i \lambda|x|}}{\sqrt{|x|}}+O\left(\frac{1}{|x|^{\frac{3}{2}}}\right) \quad \text { as }|x| \rightarrow \infty .
$$

A fact which we will not use until 6 , but which is interesting nonetheless:

Lemma 5.4. The leading singularity for the limit $x \rightarrow y$ is given by

$$
r_{0}^{\mathrm{i} / \mathrm{o}}(|x-y| ; \lambda)=-\frac{1}{2 \pi} \ln |x-y|+A+o\left(|x-y|^{2-\epsilon}\right)
$$

for $\epsilon>0$, where $A:=\gamma_{E}-\ln (2)+i / 4, \gamma_{E}$ being the Euler-Mascheroni constant. We will postpone the proof of this assertion until theorem 6.3.

Definition 5.5. Then the residual mapping $R_{0}(\lambda)=\left(\Delta+\lambda^{2}\right)^{-1}$ on $\mathbb{R}^{2}$ is simply given by the singular integral

$$
\mathrm{R}_{0}^{\mathrm{i} / \mathrm{o}}(\lambda) f(x):=\int_{\mathbb{R}^{n}} r_{0}^{\mathrm{i} / \mathrm{o}}(\lambda|x-y|) f(y) d y \in H^{2}\left(\mathbb{R}^{2}\right),
$$

for $f \in L_{\text {comp }}^{2}\left(\mathbb{R}^{2}\right)$.

Lemma 5.6. The resolvent operator for the free probelem, $\mathrm{R}_{0}^{\mathrm{i} / \mathrm{o}} \in \mathcal{I}^{-2}(\Omega ; \Lambda)$. Let $f \in L_{\text {comp }}^{2}(\Omega)$. Then the limit in infinity is given by

$$
\mathrm{R}_{0}^{\mathrm{i} / \mathrm{o}}(\lambda) f(x)=\frac{e^{\mp i \lambda|x|}}{\sqrt{|x|}} \int_{\mathbb{R}^{2}} e^{-i \lambda \omega y} f(y ; \lambda) d y+O\left(\frac{1}{|x|}\right)
$$

as $|x| \rightarrow \infty$.

Proof. Insertion of equation (5.5) into (5.8).

Will will continue our discussion of the fundamental solution's kernel in section 6.1 where we will take a closer look at the expansion for $|x-y| \rightarrow 0$.

\subsection{The Resolvent for the Problem with Bound- ary}

In this subsection we will show the existence of the resolvent operators $\mathrm{R}^{\mathrm{i} / \mathrm{o}}(\lambda)$. The proof is essentially the same as in [LP72, Theorem 5.4], but utilizes the elliptic theory of cone-degenerate differential operators when dealing with the intermediate problem (5.10).

Note that Lax and Phillips have already extended this result to non-smooth domains by themselves [LP78]. However, using our techniques from singular analysis, we are in principle able to resolve more detail when dealing with the intermediate problem (5.10) below. 
Theorem 5.7. The resolvent operator is an internal operator $\mathrm{R}^{\mathrm{i} / \mathrm{o}} \in \mathcal{I}^{-2}(\Omega ; \Lambda)$

$$
\mathrm{R}^{\mathrm{i} / \mathrm{o}}(\lambda): L_{\text {comp }}^{2}(\Omega) \rightarrow \mathcal{K}_{-\frac{1}{2}}^{s, \gamma}(\Omega), \quad \lambda \in \Lambda .
$$

(a) $\mathrm{R}^{\mathrm{i} / \mathrm{o}}(\lambda)$ is holomorphic for $\lambda \in \Lambda_{0}^{-}$,

(b) $\mathrm{R}^{\mathrm{i} / \mathrm{o}}(\lambda)$ is meromorphic on $\Lambda$.

Proof. Let $\varphi \in C_{0}^{\infty}\left(\mathbb{R}^{2}\right)$ be a cut-off function with $\varphi \equiv 1$ in a neighborhood of the obstacle and $\varphi \equiv 0$ in a neighborhood of the circle $\mathbb{S}_{\rho}^{1}=\{|x|=\rho\}$. Let $M_{\varphi}$ be the associated multiplication operator. Let $\mathrm{H}_{\zeta} \psi$ be the solution operator of the following system:

$$
\left\{\begin{aligned}
\left(\Delta+\zeta^{2}\right) h_{\zeta}=0 & \text { in } \Omega, \\
\gamma_{0}^{+} h_{\zeta}=\psi & \text { on } \partial \Omega, \\
h_{\zeta}=0 & \text { on } \mathbb{S}_{\rho}^{1} .
\end{aligned}\right.
$$

One can easily verify that $\mathrm{H}_{\zeta}$ is a linear operator. Suppose that $g \in L^{2}\left(\Omega_{\rho}\right) \subset$ $L^{2}\left(\mathbb{R}^{2}\right)$ and set:

$$
v^{\mathrm{i} / \mathrm{o}}:=\mathrm{R}_{0}^{\mathrm{i} / \mathrm{o}}(\lambda) g \in H^{2}\left(\mathbb{R}^{2}\right),
$$

and with $h_{\zeta}^{\mathrm{i} / \mathrm{o}}:=\mathrm{H}_{\zeta} \gamma_{0}^{+} v^{\mathrm{i} / \mathrm{o}}$ :

$$
u^{\mathrm{i} / \mathrm{o}}:=v^{\mathrm{i} / \mathrm{o}}-\mathrm{M}_{\varphi} h_{\zeta}^{\mathrm{i} / \mathrm{o}} .
$$

One can easily see that $u^{\mathrm{i} / \mathrm{o}}$ is a solution of the system (5.1) with homogeneous Dirichlet bondary conitions and $\mathrm{f}$ given by:

$$
\begin{aligned}
\left(\Delta+\lambda^{2}\right) u^{\mathrm{i} / \mathrm{o}} & =\left(\Delta+\lambda^{2}\right) v^{\mathrm{i} / \mathrm{o}}-\left(\Delta+\lambda^{2}\right) \varphi h_{\zeta} \\
& =g-\underbrace{\left(\Delta+\lambda^{2}\right) \varphi h_{\zeta}}_{\mathrm{T}^{\mathrm{i} / \mathrm{o}}(\lambda) g:=} .
\end{aligned}
$$

Note that $\mathrm{T}^{\mathrm{i} / \mathrm{o}}(\lambda) g$ is indeed an operator dependent on $\mathrm{g}$ because ultimately $h_{\zeta}$ is a function of $g$. Since $\left.v\right|_{\partial \Omega}$ obviously satisfies the extended compatibility conditions.

Using the well-known identity $\Delta\left(\varphi h_{\zeta}\right)=h_{\zeta} \Delta \varphi+2 \nabla \varphi \cdot \nabla h_{\zeta}+\varphi \Delta h_{\zeta}$ and $\Delta h_{\zeta}=$ $-\zeta^{2} h_{\zeta}$, we can derive

$$
\mathrm{T}^{\mathrm{i} / \mathrm{o}}(\lambda) g=2 \nabla \varphi \nabla h_{\zeta}+(\Delta \varphi) h_{\zeta}+\left(\zeta^{2}-\lambda^{2}\right) \varphi h_{\zeta} .
$$

That is, only first order differentials act on $h_{\zeta}$. We will demonstrate that $\mathrm{T}^{\mathrm{i} / \mathrm{o}}(\lambda): L^{2}\left(\Omega_{\rho}\right) \rightarrow L^{2}\left(\Omega_{\rho}\right)$ is a compact operator. In order to achieve this, we will have to analyse the solution of the system (5.10) thoroughly. $\left\|\mathrm{H}_{\zeta} \psi\right\| \leq$ $c\|\psi\|$. On the other hand, $\left\|\gamma_{0}^{+} \mathrm{R}_{0}(\lambda) g\right\| \leq c^{\prime}\left\|\mathrm{R}_{0}(\lambda) g\right\| \leq c^{\prime \prime}\|g\|$.

The solution for this problem can be written as $h=h_{\text {reg }}+h_{\text {sing }}, h_{\text {reg }} \in H^{2}\left(\Omega_{\rho}\right)$ and $h_{\text {sing }} \in H^{1+\theta}\left(\Omega_{\rho}\right)$. Hence, we may estimate:

$$
\|h\|_{1+\theta} \leq C C_{\lambda}\|g\|_{0,0} .
$$

Now $\mathrm{T}(\lambda)$ is an operator dependent on at most first derivatives of $h$ :

$$
\left\|\mathrm{T}^{\mathrm{i} / \mathrm{o}}(\zeta) g\right\|_{\theta, \gamma} \leq C\|h\|_{\theta+1, \gamma+1} \leq C_{\zeta}\|g\|_{0,0}
$$


But $\mathcal{H}^{0,0}\left(\Omega_{\rho}\right) \cong L^{2}\left(\Omega_{\rho}\right)$. Thus, $\mathrm{T}^{\mathrm{i} / \mathrm{o}}(\lambda): L^{2}\left(\Omega_{\rho}\right) \rightarrow L^{2}\left(\Omega_{\rho}\right)$ is a compact operator.

Now let $\mathfrak{I m}(\lambda) \leq 0, \lambda \neq 0$. In order to show that $\left(\mathbb{1}-\mathrm{T}^{\mathrm{i} / \mathrm{o}}(\lambda)\right)$ is invertible, suppose that $\left(\mathbb{1}-\mathrm{T}^{\mathrm{i} / \mathrm{o}}(\lambda)\right) g=0$ for a $g \in L^{2}\left(\Omega_{\rho}\right)$. According to (??), $u^{\mathrm{i} / \mathrm{o}}$ is then a solution of the reduced wave equation, i.e. $f=\left(\Delta+\lambda^{2}\right) u^{\mathrm{i} / \mathrm{o}}=0$. If $\mathfrak{I m}(\lambda)=0, \lambda \neq 0, u^{\mathrm{i} / \mathrm{o}}=0$ by Rellich's uniqueness theorem. For $\lambda \in \Lambda_{0}^{-}$, consider

$$
\left\{\begin{aligned}
(\Delta+p) z & =0, \\
z & =0,
\end{aligned}\right.
$$

where $p$ is given by

$$
p= \begin{cases}\zeta^{2} & \text { in } \Omega^{c} \\ \lambda^{2} & \text { in } \Omega_{\rho}\end{cases}
$$

One can see that $z$ is a solution of $(\Delta+p) z=0$ with $\left.p\right|_{\mathbb{S}_{\rho}^{1}}=0$. This leads to

$$
\int\left(|\nabla z|^{2}-p|z|^{2}\right) d x=0 .
$$

But since $\mathfrak{I m}\left(\zeta^{2}\right) \neq 0$ and $\mathfrak{I m}\left(\lambda^{2}\right) \neq 0$, we can infer

$$
\int_{\Omega_{\rho}}|z|^{2} d x=0 .
$$

Hence, $z \equiv 0 \Rightarrow h \equiv 0$, and finally $g \equiv 0$ due to equation (??).

It is clear by its very definition that $\mathrm{R}_{0}^{\mathrm{i} / \mathrm{o}}(\lambda)$ is a holomorphic function on $\Lambda$. That $T^{\mathrm{i} / \mathrm{o}}(\lambda)$ is holomorphic can be seen by $(5.12)$ and by acknowledging the fact that $h_{\lambda}$ is holomorphic, too. The analytic Fredholm theorem (theorem A.3.1) implies that $\left(\mathbb{1}-\mathrm{T}^{\mathrm{i} / \mathrm{o}}(\lambda)\right)^{-1}: L^{2}\left(\Omega_{\rho}\right) \rightarrow L^{2}\left(\Omega_{\rho}\right)$ is an operator-valued function which is meromorphic on $\Lambda_{-1} \backslash D$, where $D \subset \Lambda$ is a discrete subset. Now set

$$
u_{\lambda}^{\mathrm{i} / \mathrm{o}}:=\left(\mathbb{1}-\mathrm{M}_{\varphi} \mathrm{H}_{\zeta} \gamma_{0}^{+}\right) \mathrm{R}_{0}^{\mathrm{i} / \mathrm{o}}(\lambda)\left(\mathbb{1}-\mathrm{T}^{\mathrm{i} / \mathrm{o}}(\lambda)\right)^{-1} f .
$$

This function obviously solves the reduced wave equation, i.e. $\left(\Delta+\lambda^{2}\right) u_{\lambda}^{\mathrm{i} / \mathrm{o}}=0$. For $|x|>\rho$, the second term vanishes and one is left with the expression

$$
u_{\lambda}^{\mathrm{i} / \mathrm{o}}=\mathrm{R}_{0}^{\mathrm{i} / \mathrm{o}}(\lambda)\left(\mathbb{1}-\mathrm{T}^{\mathrm{i} / \mathrm{o}}(\lambda)\right)^{-1} f \quad \text { for }|x|>\rho .
$$

Both the radiation condition and the function space follow immediately. Using lemma (5.6) and setting $F:=\left(\mathbb{1}-\mathrm{T}^{\mathrm{i} / \mathrm{o}}(\lambda)\right)^{-1} f$ yields the result, i.e.

$$
u_{\lambda}^{\mathrm{i} / \mathrm{o}}(x)=\frac{1}{2 \sqrt{\lambda}} \frac{1}{\sqrt{2 \pi}} \frac{e^{ \pm i \lambda|x|}}{\sqrt{|x|}} \int_{\Omega_{\rho}} e^{\mp i \lambda \omega y} F(y ; \lambda) d y
$$

Notice that for smooth boundaries, the target space of $\mathrm{R}^{\mathrm{i} / \mathrm{o}}(\lambda)$ is $H_{\mathrm{loc}}^{2}(\Omega)$. It is only due to the appearance of singular functions that the maximal regularity is lower.

The poles of the resolvent $\mathrm{R}^{\mathrm{i} / \mathrm{o}}(\lambda)$ are directly related with the scattering poles of the scattering matrix. Our next step will be to show that the poles correspond to the zeroes of the operator $\mathrm{C}_{01}(\lambda)$ both in location and multiplicity. 


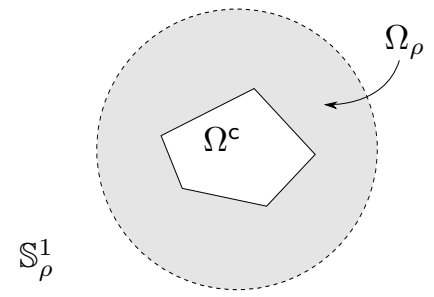

Figure 5.1: The neighborhood $\Omega_{\rho}$ around the obstacle

\subsection{Solution Operator}

This section is essentially a generalization of well-known facts to the setting with polygonal boundaries. The main purpose is to show that, for the Dirichlet problem, the scattering poles of the resolvent $\mathrm{R}^{ \pm}(\lambda)$ are identical to the poles of Calderón operator $\mathrm{C}_{01}(\lambda)$.

Let $\mathrm{E}: H^{s}(\partial \Omega) \rightarrow H^{s+\frac{1}{2}}(\Omega)$ be the extension operator, i.e. the (nonunique) operator which maps $\varphi$ to a function $\mathrm{E} \varphi \in H^{s+\frac{1}{2}}(\Omega)$ such that $\gamma^{+} \mathrm{E} \varphi=\varphi$.

Proposition 5.8. Define the operator

$$
\mathrm{K}^{\mathrm{i} / \mathrm{o}}(\lambda):=\mathrm{E}+\mathrm{R}^{\mathrm{i} / \mathrm{o}}(\lambda) \mathrm{P}(\lambda) \mathrm{E},
$$

$K^{\mathrm{i} / \mathrm{o}}$ is indeed an exact potential operator in the sense of definition?? Then for $\lambda \in \Lambda_{-1}$ the Neumann operator, $\mathrm{N}(\lambda)$, can be represented as

$$
\mathrm{N}(\lambda) \varphi=\gamma_{1}^{+} \mathrm{E} \varphi+\gamma_{1}^{+} \mathrm{R}^{\mathrm{i} / \mathrm{o}}(\lambda) \mathrm{P}(\lambda) \mathrm{E} \varphi .
$$

The Neumann operator is defined on the Riemann surface $\Lambda$, and its poles are identical with those of the Resolvent $R(\lambda)$. Finally, $K^{\mathrm{i} / \mathrm{o}}(\lambda)$ can be represented as

$$
\varphi \mapsto \mathrm{K}^{\mathrm{i} / \mathrm{o}}(\lambda) \varphi:=\mathrm{Dl}^{\mathrm{i} / \mathrm{o}}(\lambda) \varphi(x)+\mathrm{Sl}^{\mathrm{i} / \mathrm{o}}(\lambda) \mathrm{N}(\lambda) \varphi(x) .
$$

Proof. Let $\varphi \in H^{s}(\partial \Omega)$ satisfy the compatibility conditions. Consider the identity

$$
\mathrm{K}^{\mathrm{i} / \mathrm{o}}(\lambda) \gamma^{+}=\mathbb{1}+\mathrm{R}^{\mathrm{i} / \mathrm{o}}(\lambda) \mathrm{P}(\lambda) .
$$

Multiplication from the right with an extension operator $E$ yields

$$
\mathrm{K}^{\mathrm{i} / \mathrm{o}}(\lambda):=\mathrm{E}+\mathrm{R}^{\mathrm{i} / \mathrm{o}}(\lambda) \mathrm{P}(\lambda) \mathrm{E},
$$

where we have $\gamma^{+} \mathrm{E}=\mathbb{1}_{H(\partial)}$. Application of the normal vector field yields the result

$$
\gamma_{1}^{+} \mathrm{K}^{\mathrm{i} / \mathrm{o}}(\lambda)=\gamma_{1}^{+}+\gamma_{1}^{+} \mathrm{R}^{\mathrm{i} / \mathrm{o}}(\lambda) \mathrm{P}(\lambda) \mathrm{E},
$$

which is, by definition, the Dirichlet-to-Neumann operator.

The arguments given for the last part largely resemble the ones in [LP67], although the appear in a slightly different context. In order to show the last part, set $u_{\lambda}(x):=\mathrm{K}(\lambda) \varphi(x)$ for $x \in \Omega_{\rho}$ and assume that $\rho>0$ large enough such that it contains the whole scattering object.

$$
u_{\lambda}(x)=\int_{\Omega_{\rho}} \delta^{(2)}(x-y) u_{\lambda}(y) d y=\int_{\Omega_{\rho}}\left(\Delta_{y}+\lambda^{2}\right) r_{0}^{\mathrm{i} / \mathrm{o}}(\lambda|x-y|) u_{\lambda}(y) d y
$$


Since $u_{\lambda}$ is a solution of the Helmholtz equation, we may subtract the following term:

$$
0=\int_{\Omega_{\rho}} r_{0}^{\mathrm{i} / \mathrm{o}}(\lambda|x-y|)\left(\Delta_{y}+\lambda^{2}\right) u_{\lambda}(y) d y .
$$

Since equation (5.16) can be reformulated by Green's second identity,

$$
\begin{aligned}
u_{\lambda}(x)= & \int_{\Omega_{\rho}}\left[\Delta_{y} r_{0}^{ \pm}(\lambda|x-y|) u_{\lambda}(y)-r_{0}^{ \pm}(\lambda|x-y|) \Delta_{y} u_{\lambda}(y)\right] d y \\
= & \sum_{j=1}^{N} \int_{\partial \Omega_{j}}\left[\frac{\partial r_{0}^{ \pm}}{\partial \nu_{j}(y)}(\lambda|x-y|) \gamma_{0}^{+} u_{\lambda}(y)-r_{0}^{ \pm}(\lambda|x-y|) \gamma_{1}^{+} u_{\lambda}(y)\right] d s_{j}(y) \\
& -\int_{\mathbb{S}_{\rho}^{1}}\left[\frac{\partial r_{0}^{ \pm}}{\partial \nu(y)}(\lambda|x-y|) \gamma_{0}^{+} u_{\lambda}(y)-r_{0}^{ \pm}(\lambda|x-y|) \gamma_{1}^{+} u_{\lambda}(y)\right] d s_{\rho}(y)
\end{aligned}
$$

where $\partial_{\nu(y)}$ in the last line denotes the outward pointing normal vector field of $\mathbb{S}_{\rho}^{1}$. The first term is by definition $\operatorname{Dl}(\lambda) \varphi(x)+\operatorname{Sl}(\lambda) \mathrm{N}(\lambda) \varphi(x)$. The second term vanishes if we take the radiation condition into consideration and take the limit $\rho \rightarrow \infty$.

Corollary 5.9. $\mathrm{C}_{00}(\lambda)+\mathrm{C}_{01}(\lambda) \mathrm{N}(\lambda)=\mathbb{1}$.

\subsection{Scattered Plane Waves and Wave Operators}

The scattered wave obviously satisfies the Dirichlet boundary condition.

$$
\left\{\begin{aligned}
\left(\Delta+\lambda^{2}\right) v^{\mathrm{i} / \mathrm{o}} & =0, \\
\gamma_{0}^{+} v^{\mathrm{i} / \mathrm{o}} & =-e^{-i \lambda x \omega} .
\end{aligned}\right.
$$

Consider the ansatz $f(x, \omega):=-\varphi(x) e^{-i \lambda x \omega} \in H^{2}\left(\mathbb{R}^{2}\right)$, where $\varphi \in C_{c}^{\infty}\left(\mathbb{R}^{2}\right)$ is a cut-off function which is supported in $\mathbb{B}_{\rho}(0)$. Then, according to Proposition 5.8 above, the solution is given by

$$
v^{\mathrm{i} / \mathrm{o}}(x, \omega, \lambda):=f_{\omega}(x)+\mathrm{R}^{\mathrm{i} / \mathrm{o}}(\lambda) \mathrm{P}(\lambda) f_{\omega}(x) .
$$

For $|x|>0, f$ vanishes, and the second term reduces to

$$
v^{\mathrm{i} / \mathrm{o}}(x, \omega, \lambda)=\mathrm{R}_{0}^{\mathrm{i} / \mathrm{o}}(\lambda) F(x, \omega) \text { for }|x|>\rho,
$$

where $F(x, \omega, \lambda)=\left(\mathbb{1}-\mathrm{T}^{\mathrm{i} / \mathrm{o}}(\lambda)\right)^{-1} \mathrm{P}(\lambda) f(\cdot, \omega)$. Notice that $F$ inherits the meromorphic structure of the resolvent $\mathrm{R}^{\mathrm{i} / \mathrm{o}}$.

We define the incoming and outgoing scattered plane waves by

$$
\psi^{\mathrm{i} / \mathrm{o}}(x, \omega, \lambda):=\frac{1}{2 \pi}\left[(1-\varphi(x)) e^{-i \omega x \lambda}+\mathrm{R}^{\mathrm{i} / \mathrm{o}}(\lambda) \mathrm{P}(\lambda) f_{\omega}(x)\right] .
$$

Definition 5.10. Define the operators $\Phi_{\text {in }}, \Phi_{\text {out }}: L^{2}(\Omega) \rightarrow L^{2}\left(\mathbb{R}_{\xi}^{2}\right)$ by

$$
\Phi_{\mathrm{i} / \mathrm{o}} f(\omega, \sigma):=\int_{\Omega} \overline{\psi^{\mathrm{i} / \mathrm{o}}(x, \omega, \sigma)} f(x) d x .
$$


Lemma 5.11. $\Phi_{\text {in }}$ and $\Phi_{\text {out }}$ are unitary operators.

Proof. We will first prove that both operators are isometrical .

Corollary 5.12. The adjoints of the operators $\Phi_{\mathrm{i} / 0}$ are given by

$$
\Phi_{\mathrm{in}}^{*} g(x)=\int_{\mathbb{R}^{2}} \psi^{\text {in }}(x, \omega, \sigma) g(\omega, \sigma) d \omega d \sigma .
$$

Definition 5.13. The incoming and outgoing wave operators are defined by

$$
\mathrm{W}_{\text {in }}:=\Phi^{*} \Phi_{\text {in }} \quad \text { and } \quad \mathrm{W}_{\text {out }}:=\Phi^{*} \Phi_{\text {out }} .
$$

Both are unitary mappings $L^{2}(\Omega) \rightarrow L^{2}\left(\mathbb{R}_{x}^{2}\right)$.

Notice that the footnotes ' $x$ ' and ' $\xi$ ' emphasized the physical interpretation that the wave operators map into the asymptotic space with physical coordinates as opposed to Fourier space.

It is a well established fact that, for the wave equation, the definition of the scattering operator in the dynamical picture and in the static picture both coincide. We state this fact in the following proposition, but leave away the proof, since, for one, its methods go through with only slight modifications, and in addition, one could simply start with Definition 5.13 as an axiom. The interested reader may consult, for example, the monograph by Wilcox [Wil75].

Proposition 5.14. Under general considerations, with the Hamiltonians $\mathrm{H}_{0}$ and $\mathrm{H}$ defined in the obvious way,

$$
\begin{aligned}
\mathrm{W}_{\mathrm{in}} & =\underset{t \rightarrow \infty}{\mathrm{s}-\lim } e^{i t \sqrt{\mathrm{H}_{0}}} \mathrm{~J}_{\Omega} e^{-i t \sqrt{\mathrm{H}_{0}},} \\
\mathrm{~W}_{\text {out }} & =\underset{t \rightarrow-\infty}{\mathrm{s}-\lim } e^{i t \sqrt{\mathrm{H}_{0}}} \mathrm{~J}_{\Omega} e^{-i t \sqrt{\mathrm{H}_{0}},}
\end{aligned}
$$

with $\mathrm{J}_{\Omega}: L^{2}(\Omega) \rightarrow L^{2}\left(\mathbb{R}^{2}\right)$ being the embedding through extension by zero on $\Omega^{\mathrm{c}}$.

\subsection{The S-Matrix and Scattering Poles}

Return to our discussion in section 2.2.3 and recall that the Scattering operator is defined by:

$$
\mathrm{S}:=\mathrm{W}_{\text {out }} \mathrm{W}_{\text {in }}^{*} .
$$

As a simple consequence, the Fourier transform of the scattering operator is given by

$$
\hat{\mathrm{S}}=\Phi \mathrm{S} \Phi^{*}=\Phi_{\text {out }} \Phi_{\text {in }}^{*} .
$$

In order to achieve a representation of the scattering operator as a direct integral, we need to apply Proposition 2.2. Therefore, we need the following lemma:

Lemma 5.15. Let $\varphi \in C_{0}(\mathbb{R})$ be an even function. Then

$$
\varphi(|\xi|) \hat{\mathrm{S}}=\hat{\mathrm{S}} \varphi(|\xi|) .
$$

Consequently, $\hat{\mathrm{S}}$ can be written as a direct integral, i.e.

$$
\hat{\mathrm{S}}=\sum_{\sigma \in \mathbb{R}_{+}} \hat{\mathrm{S}}(\sigma) .
$$


Given these prerequisites, we may prove the main theorem of this section:

Theorem 5.16. The modified scattering matrix is of the form

$$
\hat{\mathrm{S}}(\sigma)=\mathbb{1}+\left(\frac{\sigma}{2 \pi i}\right)^{\frac{1}{2}} \mathrm{~A}(\sigma)
$$

where $A(\sigma)$ is a bounded operator $L^{2}\left(\mathbb{S}^{1}\right) \rightarrow L^{2}\left(\mathbb{S}^{1}\right)$ given by:

$$
\hat{\mathrm{S}}(\sigma) \hat{f}^{\mathrm{in}}(\omega)=\hat{f}^{\mathrm{in}}(\omega)+\left(\frac{\sigma}{2 \pi i}\right)^{\frac{1}{2}} \int_{\mathbb{S}^{1}} \overline{s^{\mathrm{in}}(-\theta, \omega, \sigma)} \hat{f}^{\mathrm{in}}(\theta) d \theta .
$$

The Schwartz kernel depends on the scattered wave $v^{\text {in }}$ by

$$
s^{\text {in }}(\theta, \omega, \sigma):=\lim _{r \rightarrow \infty} r^{\frac{1}{2}} e^{-i \sigma r} v^{\text {in }}(r \theta, \omega, \sigma) .
$$

Proof. Set

$$
w_{\sigma}(y, \omega):=\psi_{\sigma}^{\text {out }}-\psi_{\sigma}^{\text {in }}=v_{\sigma}^{\text {out }}-v_{\sigma}^{\text {in }} .
$$

We will demonstrate that the assertion is equivalent to

$$
w(y, \sigma \omega)=-\left(\frac{\sigma}{2 \pi i}\right)^{\frac{1}{2}} \int_{\mathbb{S}_{\rho}^{1}} \overline{s^{\text {in }}(\omega, \theta, \sigma)} \psi^{\text {out }}(y, \theta, \sigma) d \theta .
$$

Recall that $\mathrm{S}=\Phi_{\text {out }} \Phi_{\text {in }}^{*}$. By definition of $\Phi_{\mathrm{i} / \mathrm{o}}$, one quickly calculates

$$
\mathrm{S} \psi_{\sigma}^{\text {in }}=\psi_{\sigma}^{\text {out }}
$$

Now observe that $\left(\Delta_{y}+\sigma^{2}\right) w_{\sigma}(y, \omega)=0$ and $\gamma_{0}^{+} w_{\sigma}=0$. As in (??), we may express $w_{\sigma}$ with the help of the third Green identity for polyhedral domains (corollary A.1.6) as:

$w_{\sigma}(x, \omega)=-\int_{\mathbb{S}_{\rho}^{1}}\left[\gamma_{0}^{+} w_{\sigma}(y, \omega) \partial_{r} r_{0}^{\mathrm{i} / \mathrm{o}}(\sigma|x-y|)-\gamma_{1}^{+} w_{\sigma}(y, \omega) r_{0}^{\mathrm{i} / \mathrm{o}}(\sigma|x-y|)\right] d s(y)$.

since $\partial_{\nu(y)}=-\partial_{r}$ on $\mathbb{S}_{\rho}^{1}$. Now, as $r \rightarrow \infty$,

$$
w_{\sigma}(x, \omega)=\left(\frac{\sigma}{2 \pi i}\right)^{\frac{1}{2}} \frac{1}{r^{\frac{1}{2}}}\left[e^{i \sigma r}-e^{-i \sigma r}\right]+O\left(\frac{1}{r^{\frac{3}{2}}}\right)
$$

and

$$
\partial_{r} w_{\sigma}(x, \omega)=i \sigma\left(\frac{\sigma}{2 \pi i}\right)^{\frac{1}{2}} \frac{1}{r^{\frac{1}{2}}}\left[e^{i \sigma r}-e^{-i \sigma r}\right]+O\left(\frac{1}{r^{\frac{3}{2}}}\right) .
$$

Together with the asymptotics (5.5) and (5.6) this yields for the integrand

$$
\overline{s^{\text {in }}(-\theta, \omega, \sigma)} \psi^{\text {out }}(y, \theta, \sigma) r^{-1}+O\left(r^{-2}\right) \quad \text { as } r \rightarrow \infty \text {. }
$$

Evaluating the integral in radial coordinates yields the measure $r d \theta$. Inserting these expressions into (??) yields

$$
\int_{\mathbb{S}_{\rho}^{1}}\left[w_{\sigma}(y, \omega) \partial_{r} r_{-} \partial_{r} w_{\sigma}(y, \omega)\right] d s(y)+o(1) \quad \text { as } r \rightarrow \infty .
$$


By Lemma 5.6, where

$$
s^{\text {in }}(\theta, \omega, \lambda)=\frac{1}{4 \pi}\left(\frac{\lambda}{2 \pi i}\right)^{\frac{n-3}{2}} \int_{\Omega_{\rho}} e^{-i \lambda \theta \cdot y} F(y, \omega, \lambda) d y .
$$

Therefore, we obtain as a corollary:

Corollary 5.17. The scattering matrix $\hat{\mathrm{S}}(\sigma)$ has an analytical continuation to the logarithmic Riemann surface $\Lambda$. It is holomorphic on the lower half of the principal sheet, i.e. on $\Lambda_{0}^{-}$, and meromorphic everywhere else, the poles being the discrete subset $D$ from Theorem 5.7. 


\section{Chapter 6}

\section{Mapping Properties of the Layer Potentials}

This chapter deals with the layer potentials in the case of polygonal boundaries. This means that we will recast them as potential operators in the sense of Definition 4.4 and, by understanding their symbolic structure, show that they can be continuously extended to continuous operators,

$$
\mathrm{Sl}^{\mathrm{i} / \mathrm{o}}(\lambda): \mathcal{H}^{s, \gamma}(\partial \Omega) \rightarrow \mathcal{K}_{\mathrm{i} / \mathrm{o}}^{s+\frac{3}{2}, \gamma+\frac{3}{2}}(\Omega) \quad \lambda \in \Lambda_{0}^{ \pm}
$$

and

$$
\mathrm{Dl}^{\mathrm{i} / \mathrm{o}}(\lambda): \mathcal{H}^{s, \gamma}(\partial \Omega) \rightarrow \mathcal{K}_{\mathrm{i} / \mathrm{o}}^{s+\frac{1}{2}, \gamma+\frac{1}{2}}(\Omega) \quad \lambda \in \Lambda_{0}^{ \pm}
$$

Equally important are their traces on the boundary, both from the outside and the inside of the scattering obstacle. However, as it has been demonstrated in [Kre99], the jump relations have to be modified for polygonal obstacles. We are able to derive the same results by an application of Mellin pseudodifferential methods. We will see that these have to be modified in the case of the double layer potential for polygonal domains. As a reminder, we will state these relation for the case of a smooth boundary:

Theorem 6.1 (Jump relations for smooth boundaries). Assume that the boundary $\partial \Omega$ of the exterior domain is smooth. Let $\varphi \in H^{s}(\partial \Omega), s \geq 0$. Then the following jump relations hold:

$$
\begin{aligned}
\gamma_{0}^{+} \operatorname{Sl}(\lambda) \varphi & =\gamma^{-} \operatorname{Sl}(\lambda) \varphi, & \gamma_{1}^{ \pm} \operatorname{Sl}(\lambda) \varphi & = \pm \frac{1}{2} \varphi-\frac{1}{2} \varphi \\
\gamma_{0}^{ \pm} \operatorname{Dl}(\lambda) \psi & = \pm \frac{1}{2} \psi-\frac{1}{2} \mathrm{~N}(\lambda) \psi, & \gamma_{1}^{ \pm} \operatorname{Dl}(\lambda) \psi & = \pm \frac{1}{2} \psi-\frac{1}{2} \mathrm{~N}(\lambda) \psi .
\end{aligned}
$$

The boundary limits of the layer potentials are the Calderón projectors $\mathrm{C}_{00}(\lambda)$ and $\mathrm{C}_{01}(\lambda)$. We will prove that these operators are elements of the calculus $\mathcal{C}^{\mu}(\partial \Omega, \mathbf{g} ; \Lambda)$, with $\mu=-1$ and $\mathbf{g}=(\gamma, \gamma+1, \Theta)$ for the single layer potential and $\mu=0,(\gamma, \gamma, \Theta)$ for the double layer potential. The latter proves that the boundary limits of the double layer potential are not compact with respect to the weighted Sobolev spaces $\mathcal{H}^{s, \gamma}(\partial \Omega)$. 


\subsection{The Layer Potentials}

The realization of the operator $\mathrm{K}^{\mathrm{i} / \mathrm{o}}(\lambda)$ is somewhat more difficult and requires the use of the so-called layer potentials. We are seeking a realization of the form

$$
\mathrm{K}^{\mathrm{i} / \mathrm{o}}(\lambda) g(x)=\mathrm{Dl}^{\mathrm{i} / \mathrm{o}}(\lambda) \varphi(x)+\mathrm{Sl}^{\mathrm{i} / \mathrm{o}}(\lambda) \psi(x), \quad x \in \Omega \cup \Omega^{\mathrm{c}} .
$$

We initially define for $\varphi, \psi \in \prod_{j=1}^{N} C_{c}^{\infty}\left(\partial \Omega_{j}\right)$ the potential operator

$$
\mathrm{K}^{\mathrm{i} / \mathrm{o}}(\lambda):\left[\begin{array}{l}
\varphi \\
\psi
\end{array}\right] \mapsto \mathrm{Dl}^{\mathrm{i} / \mathrm{o}}(\lambda) \varphi(x)+\mathrm{Sl}^{\mathrm{i} / \mathrm{o}}(\lambda) \psi(x) .
$$

Definition 6.2. For $\varphi, \psi \in \prod_{j=1}^{N} C_{c}^{\infty}\left(\partial \Omega_{j}\right), \varphi=\left(\varphi_{j}\right)_{j=1}^{N}$ and $\psi=\left(\psi_{j}\right)_{j=1}^{N}$, we define the single layer and double layer potentials as the integral operators

$$
\mathrm{Si}^{\mathrm{i} / \mathrm{o}}(\lambda) \psi(x):=\sum_{j=1}^{N} \int_{\partial \Omega_{j}} r_{0}^{\mathrm{i} / \mathrm{o}}(\lambda|x-y|) \psi_{j}(y) d s_{j}(y), x \in \Omega \cup \Omega^{\mathrm{c}}
$$

and

$$
\mathrm{Dl}^{\mathrm{i} / \mathrm{o}}(\lambda) \varphi(x):=\sum_{j=1}^{N} \int_{\partial \Omega_{j}} \frac{\partial}{\partial \nu_{j}(y)}\left(r_{0}^{\mathrm{i} / \mathrm{o}}(\lambda|x-y|)\right) \varphi_{j}(y) d s_{j}(y), x \in \Omega \cup \Omega^{\mathrm{c}},
$$

respectively.

Notice that both layer potentials are defined in the interior as well as in the exterior of the obstacle, in other words, in both connected components of $\Omega \cup \Omega^{c}$. It is of importance to define the traces of both $\operatorname{Sl}(\lambda) \varphi$ and $\operatorname{Dl}(\lambda) \psi$ on the interior and exterior side of the boundary $\partial \Omega$. We shall however postpone this until later.

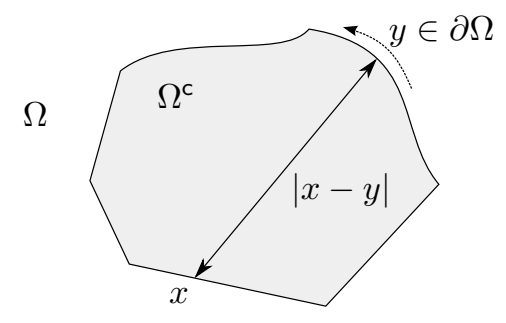

Figure 6.1: Integrating along $\partial \Omega$

Having defined the layer potentials, we may now set Since our main concern is the Dirichlet problem (5.1), we are exclusively interested in the operators in the first line, that is

$$
\mathrm{C}_{00}(\lambda) \psi=\gamma_{0}^{+} \operatorname{Dl}(\lambda) \psi \quad \text { and } \quad \mathrm{C}_{01}(\lambda) \varphi=\gamma_{0}^{+} \operatorname{Sl}(\lambda) \varphi .
$$

The other two Calderón operators are

$$
\mathrm{C}_{10}(\lambda) \psi=\gamma_{1}^{+} \operatorname{Dl}(\lambda) \psi, \quad \text { and } \quad \mathrm{C}_{11}(\lambda) \varphi=\gamma_{1}^{+} \operatorname{Sl}(\lambda) \varphi .
$$


It will be one of our tasks in chapter 6 to prove that $K^{\mathrm{i} / \mathrm{o}}(\lambda)$ can be extended to a mapping

$$
\mathrm{K}^{\mathrm{i} / \mathrm{o}}(\lambda): \mathcal{H}^{s, \gamma}(\partial \Omega) \rightarrow \mathcal{K}_{\mathrm{i} / \mathrm{o}}^{s+\frac{1}{2}, \gamma}(\Omega) .
$$

The function space is defined in (3.15). This will be the subject of chapter 6 . We continue the investigation of the fundamental solution's properties.

In order to simplify notation on the following pages, we define the entire function $^{1}$

$$
F(z):=\sum_{k=0}^{\infty} a_{k} \frac{(-1)^{k}}{(k !)^{2}}\left(\frac{z}{2}\right)^{2 k}
$$

where $a_{k}=\sum_{m=1}^{k} 1 / m$ and $\gamma_{\mathrm{E}}$ is the Euler-Mascheroni constant. This function converges.

Theorem 6.3. The single layer potential may be rewritten as

$$
\mathrm{Sl}^{\mathrm{i} / \mathrm{o}}(\lambda)=-\frac{1}{2 \pi}\left(\mathrm{T}_{\log }(\lambda)+\mathrm{T}_{\mathrm{reg}}(\lambda)\right)
$$

where the kernels of the corresponding integral operators are given by $t_{\log }(\mid x-$ $y \mid, \lambda)$ and $t_{\mathrm{reg}}(|x-y|, \lambda)$ with

$$
t_{\log }(\tau, \lambda):=\ln (\tau) J_{0}(\lambda \tau)
$$

and

$$
t_{\mathrm{reg}}(\tau, \lambda):=\kappa \cdot J_{0}(\lambda \tau)-\frac{1}{2} F(\lambda \tau)+\ln (\lambda) J_{0}(\lambda \tau),
$$

Here, $\kappa:=\gamma_{E}-\ln (2)+i / 4$ is a complex constant, $\left\{a_{k}\right\}_{k \in \mathbb{N}}$ the sequence of partial sums of the harmonic series. ${ }^{2}$

Proof. The Hankel function of order zero may be written as

$$
\frac{i}{4} H_{0}^{(1)}(\lambda \tau)=-\frac{1}{4} Y_{0}(\lambda \tau)+\frac{i}{4} J_{0}(\lambda \tau)
$$

where $Y_{0}$ is the Neumann function of order zero. ${ }^{3}$ Notice, that the Neumann function may be expanded as (treating the product $\lambda \tau$ as one variable):

$$
Y_{0}(\lambda \tau)=\frac{2}{\pi}\left[\ln \left(\frac{\lambda \tau}{2}\right)+\gamma_{\mathrm{E}}\right] J_{0}(\lambda \tau)-\frac{2}{\pi} F(\lambda \tau) .
$$

Adding $(i / 4) J_{0}(\lambda \tau)$ gives the desired result.

Finally, we will discuss the relation of the fundamental solution on different sheets of the logarithmic Riemann surface.

Corollary 6.4. Let $\tau, \lambda \in \mathbb{C}, m \in \mathbb{Z}$. Then

$$
\mathrm{Sl}^{\mathrm{i} / \mathrm{o}}\left(e^{i m \pi} \lambda\right)=\mathrm{Sl}^{\mathrm{i} / \mathrm{o}}(\lambda)+i \frac{m}{2} T(\lambda)
$$

with

$$
T(\lambda) \varphi(x):=\sum_{j=1}^{N} \int_{\partial \Omega_{j}} J_{0}(\lambda|x-y|) \varphi_{j}(y) d s_{j}(y) .
$$

\footnotetext{
${ }^{1}$ Note that this function, although it occurs in the asymptotic expansion of the Hankel function, is to the author's knowledge not a canonical special function. We have only introduced this notation for convenience.

${ }^{2} \gamma_{\mathrm{E}}:=\lim _{n \rightarrow \infty}\left(-\ln (n)+\sum_{k=1}^{n} \frac{1}{k}\right)=0.57721 \ldots$

${ }^{3}$ The Neumann function is also sometimes called Bessel functions of the second kind.
} 
Proof. It is a well-known fact, that

$$
H_{0}^{(i)}\left(e^{i m \pi} \lambda \tau\right)=H_{0}^{(i)}(\lambda \tau)-i 2 m \cdot J_{0}(\lambda \tau) .
$$

Recall that both Hankel functions are related by

$$
H_{0}^{(1)}(\lambda \tau)=H_{0}^{(2)}(\lambda \tau)+2 i J_{0}(\lambda \tau), \quad \tau>0,
$$

where $J_{0}(z)$ is a Bessel function of the first kind and order zero.

One can see this by checking $J_{0}\left(e^{i m \pi} \lambda \tau\right)=J_{0}(\lambda \tau)$ and $F\left(e^{i m \pi} \lambda \tau\right)=F(\lambda \tau)$. For the middle term in (6.6) we simply use the fact that $\ln \left(e^{i m \pi} \lambda\right)=\ln (\lambda)-m$. The result follows immediately.

Analogously to Theorem 6.3 we claim:

Theorem 6.5. The double layer potential may be rewritten as

$$
\mathrm{Dl}^{\mathrm{i} / \mathrm{o}}(\lambda)=\frac{1}{2 \pi}\left(\tilde{T}_{\text {sing }}+\tilde{\mathrm{T}}_{\log }(\lambda)+\tilde{\mathrm{T}}_{\mathrm{reg}}(\lambda)\right) .
$$

where the kernels of the corresponding integral operators are given by $\tilde{t}_{\text {sing }}(x-y)$, $\tilde{t}_{\log }(x-y, \lambda)$, and $\tilde{t}_{\mathrm{reg}}(x-y, \lambda)$ :

$$
\begin{gathered}
\tilde{t}_{\text {sing }}(x-y):=\frac{\nu_{j}(y) \cdot(x-y)}{|x-y|^{2}}, \\
\tilde{t}_{\log }(x-y, \lambda):=\left.\lambda \ln (\tau) \frac{1}{\tau} J_{0}^{\prime}(\lambda \tau)\right|_{\tau=|x-y|} \nu_{j}(y) \cdot(x-y),
\end{gathered}
$$

and

$$
\tilde{t}_{\mathrm{reg}}(x-y, \lambda):=\left[\frac{1}{\tau} t_{\mathrm{reg}}^{\prime}(\tau, \lambda)+\frac{1}{\tau^{2}}\left(J_{0}(\lambda \tau)-1\right)\right]_{\tau=|x-y|} \nu_{j}(y) \cdot(x-y) .
$$

Proof. By the chain rule, we can calculate the kernel of the double layer potential as

$$
\frac{\partial}{\partial \nu_{j}(y)}\left(r_{0}^{\mathrm{i} / \mathrm{o}}(|x-y| ; \lambda)\right)=-\left.r_{0}^{\mathrm{i} / \mathrm{o} \prime}(\tau ; \lambda) \frac{\nu_{j}(y) \cdot(x-y)}{\tau}\right|_{\tau=|x-y|} .
$$

By equations (6.5) and (6.6) we have

$$
\left(r_{0}^{\mathrm{i} / \mathrm{o}}\right)^{\prime}(\tau, \lambda)=-\frac{1}{2 \pi}\left(t_{\log }^{\prime}(\tau ; \lambda)+t_{\text {reg }}^{\prime}(\tau ; \lambda)\right)
$$

with

$$
t_{\log }^{\prime}(\tau, \lambda)=\frac{1}{\tau} J_{0}(\lambda \tau)+\lambda \ln (\tau) J_{0}^{\prime}(\lambda \tau)
$$

and

$$
t_{\text {reg }}^{\prime}(\tau, \lambda)=\lambda \kappa J_{0}^{\prime}(\lambda \tau)-\lambda \frac{1}{2} F^{\prime}(\lambda \tau)+\lambda \ln (\lambda) J_{0}^{\prime}(\lambda \tau) .
$$

We consider the variable $\tau>0$ in the denominator together with $r^{\mathrm{i} / \mathrm{o} \prime}(\tau ; \lambda)$, i.e.

$$
t_{\log }^{\prime}(\tau, \lambda) \frac{1}{\tau}=\frac{1}{\tau^{2}} J_{0}(\lambda \tau)+\lambda \ln (\tau) \frac{1}{\tau} J_{0}^{\prime}(\lambda \tau)
$$


and

$$
t_{\text {reg }}^{\prime}(\tau, \lambda) \frac{1}{\tau}=\lambda \kappa \frac{1}{\tau} J_{0}^{\prime}(\lambda \tau)-\frac{\lambda}{2} \frac{1}{\tau} F^{\prime}(\lambda \tau)+\ln (\lambda) \frac{1}{\tau} J_{0}^{\prime}(\lambda \tau) .
$$

It is easy to see by their series expansions that multiplying $J_{0}^{\prime}(\lambda \tau)$ and $F^{\prime}(\lambda \tau)$ with $\tau^{-1}$ yields smooth functions in $\tau$. Hence $\tau^{-1} t_{\text {reg }}^{\prime}(\tau ; \lambda)$ is smooth in $\tau$. On the other hand, the first term of equation (6.11), $J_{0}(\lambda \tau) \tau^{-2}$, has a singularity of order $\sim \tau^{-2}$. The second term has a logarithmic singularity, more precisely, a factor of the form $\ln (\tau)$.

Taking into account the the sign in the above equation yields the decomposition

$$
\frac{\partial}{\partial \nu_{j}(y)}\left(r_{0}^{\mathrm{i} / \mathrm{o}}(|x-y| ; \lambda)\right)=\frac{1}{2 \pi}\left(\tilde{t}_{\mathrm{sing}}(x-y)+\tilde{t}_{\log }(x-y, \lambda)+\tilde{t}_{\mathrm{reg}}(x-y, \lambda)\right),
$$

where

$$
\begin{gathered}
\tilde{t}_{\text {sing }}(x-y):=\frac{\nu_{j}(y) \cdot(x-y)}{|x-y|^{2}}, \\
\tilde{t}_{\log }(x-y, \lambda):=\left.\lambda \ln (\tau) \frac{1}{\tau} J_{0}^{\prime}(\lambda \tau)\right|_{\tau=|x-y|} \nu_{j}(y) \cdot(x-y),
\end{gathered}
$$

and

$$
\tilde{t}_{\mathrm{reg}}(x-y, \lambda):=\left[\frac{1}{\tau} t_{\mathrm{reg}}^{\prime}(\tau, \lambda)+\frac{1}{\tau^{2}}\left(J_{0}(\lambda \tau)-1\right)\right]_{\tau=|x-y|} \nu_{j}(y) \cdot(x-y) .
$$

Corollary 6.6. Let $\tau, \lambda \in \mathbb{C}, m \in \mathbb{Z}$. Then

$$
\operatorname{Dl}\left(e^{i m \pi} \lambda\right)=\operatorname{Dl}(\lambda)+i \frac{m}{2} T^{\prime}(\lambda)
$$

with

$$
T^{\prime}(\lambda) \varphi(x):=\sum_{j=1}^{N} \int_{\partial \Omega_{j}} J_{0}^{\prime}(\lambda|x-y|) \varphi(y) d s_{j}(y) .
$$

Proof. One can either take the identity (6.9), substitute $\tau$ with $|x-y|$, and apply the derivative $\partial_{\nu(y)}$. Alternatively, one can check directly that $F^{\prime}\left(e^{i \pi m} \lambda\right)=$ $e^{i \pi m} F^{\prime}(\lambda), J_{0}^{\prime}\left(e^{i \pi m} \lambda\right)=e^{i \pi m} J_{0}^{\prime}(\lambda)$. These functions are each multiplied with the factor $e^{i \pi m} \lambda$. 


\subsection{The Single Layer Potential}

Theorem 6.7. The single layer potential $\mathrm{Sl}^{\mathrm{i} / \mathrm{o}}$ is a potential operator of order -1. More precisely, $\mathrm{Sl}^{\mathrm{i} / \mathrm{o}} \in \mathcal{P}^{-1}(\Omega ; \Lambda)$ with

$$
\mathrm{Sl}^{\mathrm{i} / \mathrm{o}}(\lambda): \mathcal{H}^{s, \gamma}(\partial \Omega) \rightarrow \mathcal{K}_{\mathrm{i} / \mathrm{o}}^{s+\frac{3}{2}, \gamma+\frac{3}{2}}(\Omega), \quad \lambda \in \Lambda_{0}^{ \pm}
$$

More specifically, $\mathrm{Sl}(\lambda)$ may be written as the sum

$$
\mathrm{Sl}^{\mathrm{i} / \mathrm{o}}(\lambda)=-\frac{1}{2 \pi}\left\{\mathrm{T}_{\log }(\lambda)+\mathrm{T}_{\mathrm{reg}}(\lambda)\right\},
$$

where $\mathrm{T}_{\log } \in \mathcal{P}^{-1}(\Omega ; \Lambda)$ and $\mathrm{T}_{\text {reg }} \in \mathcal{P}^{-\infty}(\Omega ; \Lambda)$. Here,

$$
\mathrm{T}_{\log }(\lambda)=\sum_{j=1}^{N} \Phi_{j}^{\prime} r \mathrm{op}_{\mathrm{M}}\left[\bar{h}_{\log , j}\right] \phi_{j}+\sum_{j=1}^{N} \Theta_{j}^{\prime} \mathrm{op}_{\psi}\left[h_{j}^{\psi}\right] \theta_{j}
$$

with the Mellin symbols given by the pairs

$$
\bar{h}_{\log , j}(\varphi, \lambda, z)=\left(h^{\alpha_{j}}(\varphi, \lambda, z), h^{0}(\varphi, \lambda, z)\right) \in M_{P}^{-1} \oplus M_{P}^{-1},
$$

where $h^{\vartheta}(\varphi, \lambda, z)=h_{0}(\varphi-\vartheta, z) \tilde{h}(\lambda, z)$ with

$$
h_{0}(\varphi, z):=\pi \frac{\cos ((\pi-\varphi) z)}{\sin (\pi z)}
$$

and

$$
\tilde{h}(\lambda, z):=\sum_{k=0}^{\infty}(-1)^{k} \lambda^{2 k} \frac{(2 k) !}{2^{2 k}(k !)^{2}} \prod_{j=1}^{2 k+1} \frac{1}{z-j} .
$$

The full symbols for the edges are $h_{j}^{\psi} \in S_{\mathrm{cl}}^{-1}\left(I_{j} \times I_{j} \times \mathbb{R}\right), I_{j} \subset \partial \Omega_{j}$.

Proof. We will first prove that each operator $\mathrm{T}_{\log }(\lambda)$ is a continuous operator: Recalling that

$$
\mathrm{Sl}^{\mathrm{i} / \mathrm{o}}(\lambda)=-\frac{1}{2 \pi}\left\{\mathrm{T}_{\log }(\lambda)+\mathrm{T}_{\mathrm{reg}}(\lambda)\right\},
$$

we focus out attention onto the first term with logarithmic kernels. Its expansion is

$$
\mathrm{T}_{\log }(\lambda)=\frac{1}{2 \pi} \sum_{k=0}^{M} \frac{(-1)^{k}}{(k !)^{2}}\left(\frac{\lambda}{2}\right)^{2 k} \mathrm{~T}_{\log , k} .
$$

The kernel of the integral operators $\mathrm{T}_{k, \log }$ are given by $t_{\log , k}(|x-y|)=\mid x-$ $\left.y\right|^{2 k} \ln (|x-y|)$. They can be regarded as a linear combination of generalized potentials in the sense of Duduchava [Dud99]. That is, we consider

$$
|x-y|^{2 k} \ln (|x-y|)=\sum_{j=1}^{k}(x-y)^{2 \beta_{j}} \ln (|x-y|),
$$

where $\beta_{j} \in \mathbb{N}_{0}^{2}$ with $\left|\beta_{j}\right|=k$. Let $E: \mathcal{H}^{s, \gamma}(\partial \Omega) \rightarrow \mathcal{H}^{s+\frac{1}{2}, \gamma+\frac{1}{2}}(\Omega)$ be the extension operator. Consider

$$
E \varphi \mapsto \int_{\Omega} F_{A}(x, y ; \lambda) E \varphi(y) d y
$$


This (pseudodifferential) operator is continuous as $\mathcal{H}^{s, \gamma}(\Omega) \rightarrow \mathcal{H}^{s+2 k, \gamma}(\Omega)$, and $\varphi \mapsto E \varphi$ is continuous. Hence,

$$
\mathrm{T}_{\log , k}: \mathcal{H}^{s, \gamma}(\partial \Omega) \rightarrow \mathcal{H}^{s+2 k+\frac{1}{2}, \gamma+2 k+\frac{1}{2}}(\Omega)
$$

is continuous. Having established the continuity of the family of operators, we proceed with establishing their pseudodifferential nature in the sense of Definition 4.4. Set

$$
\mathcal{R}_{1}:=\left(1-\sum_{j=1}^{N}\left(\Phi_{j}^{\prime}+\Theta_{j}^{\prime}\right)\right) \tilde{\mathrm{T}}_{\log } .
$$

$\mathcal{R}_{1}$ is regularizing and vanishes in a neighborhood of the boundary. Due to our choice of cut-off functions, we can reduce each localized term to one of the model cases, i.e. either a cone $X_{\theta}^{\wedge}$ or a half-space $\mathbb{R}_{+} \times \Omega^{\prime}, \Omega^{\prime} \subset \mathbb{R}$. We will only consider the localization in a corner since the interior of the edges are covered by the standard theory of pseudodifferential operators. Choose a vertex $v_{j}$ and a cut-off function $\hat{\phi}_{j}$, and consider

$$
\Phi_{j}^{\prime} \mathrm{T}_{\log }(\lambda)=\Phi_{j}^{\prime} \mathrm{T}_{\log }(\lambda) \hat{\phi}_{j}+\Phi_{j}^{\prime} \mathrm{T}_{\log }(\lambda)\left(1-\hat{\phi}_{j}\right)
$$

In the vicinity of the corner $v_{j}$ there are only two relevant terms, namely $\Phi_{j}^{j+1} \mathrm{~T}_{\log } \phi_{j}^{j}$ and $\Phi_{j} \mathrm{~T}_{\log } \phi_{j}^{j}$. Consider only the first term, i.e. $\Phi_{j}^{\prime} \mathrm{T}_{k} \phi_{j}^{j+1}$. Consider an affine transformation of the form

$$
z \mapsto e^{i \theta_{j}} z-v_{j}
$$

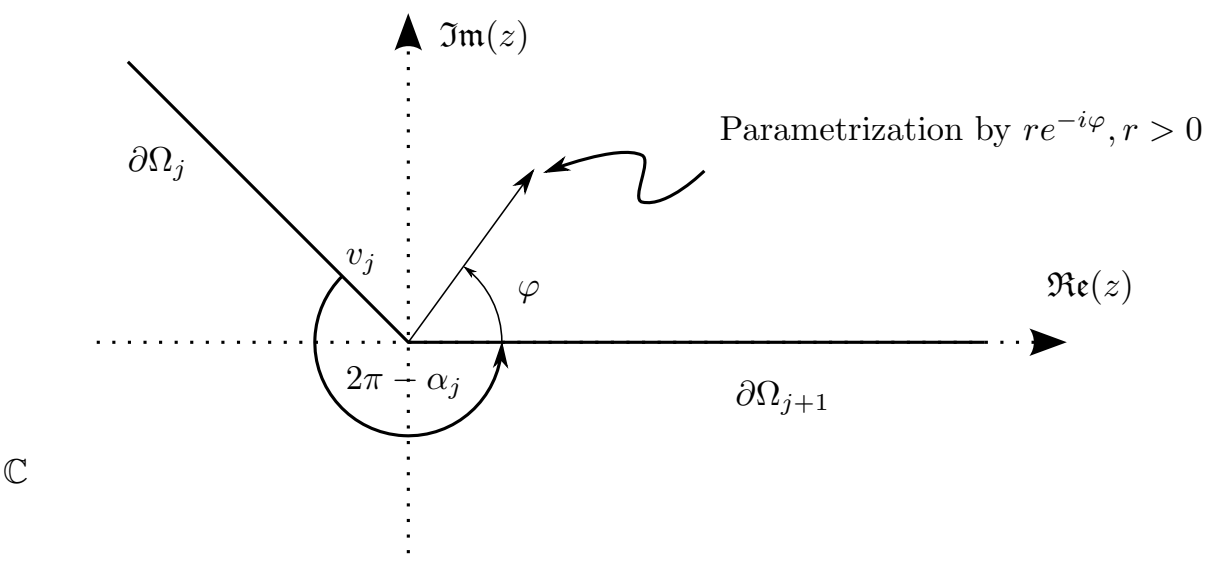

Figure 6.2: Local parametrization in the complex plane

We now make use of the fact that $\mathbb{R}^{2} \cong \mathbb{C}$ and parametrize the edges according to figure 6.2. That is, we set $x=r e^{i \varphi}$ and $y=s$ :

$$
\Phi_{j} \mathrm{~T}_{\log } \phi_{j}^{j+1}=\Phi_{j}\left(r e^{i \varphi}\right) \int_{0}^{\infty} \ln \left|r e^{i \varphi}-s\right| J_{0}\left(\lambda\left|r e^{i \varphi}-s\right|\right) \phi_{j}^{j+1} f(s) d s .
$$

Notice that the interior of the scattering obstacle is parametrized by $0<\vartheta<\alpha_{j}$, the exterior domain by $\alpha_{j}<2 \pi-\alpha_{j}$. Due to the identity

$$
t_{\log }(|x-y| ; \lambda)=\ln \left|\frac{x}{y}-1\right| J_{0}(\lambda|x-y|)+\underbrace{\ln |y| J_{0}(\lambda|x-y|)}_{\text {negligible }},
$$


we may neglect the second term. Recall that $t_{\log }(\tau, \lambda)$ is defined as the series

$$
t_{\log }(\tau ; \lambda)=\sum_{k=0}^{\infty} \frac{(-1)^{k}}{(k !)^{2}}\left(\frac{\lambda}{2}\right)^{2 k} \ln (\tau) \tau^{2 k} .
$$

Using complex radial coordinates, we can calculate:

$$
\begin{aligned}
t_{\log , \mathrm{k}}\left(r e^{i \vartheta}-s, \lambda\right) & =s^{2 k} \ln \left(\left|\frac{r}{s} e^{i \varphi}-1\right|\right)\left|\frac{r}{s} e^{i \varphi}-1\right|^{2 k} \\
& =r^{2 k+1}\left(\frac{r}{s}\right)^{-(2 k+1)} \ln \left(\left|\frac{r}{s}-e^{-i \vartheta}\right|\right)\left|\frac{r}{s}-e^{-i \varphi}\right|^{2 k} \frac{1}{s} .
\end{aligned}
$$

Set

$$
l_{k}(\sigma, \varphi):=\sigma^{-(2 k+1)} t_{\log , k}\left(\sigma-e^{i \varphi}\right) .
$$

Having rewritten the operator as a Mellin convolution operator, we will apply a Mellin transformation onto its convolution kernel (cf. example 3.25). Set, $\varphi \in[-\pi, \pi]$

$$
l_{k}(\sigma, \varphi):=\sigma^{-(2 k+1)}\left|\sigma-e^{i \varphi}\right|^{2 k} \ln \left|\sigma-e^{i \varphi}\right| .
$$

The formula itself can be calculated by using

$$
\left|t-e^{i \varphi}\right|=\sqrt{t^{2}+2 t \cos (\pi-\varphi)+1}
$$

applying integration by parts multiple times, and evaluating the integral [GR07, formula $(4.296,3)], m \in \mathbb{N}_{0}$ :

$$
\frac{1}{2} \int_{0}^{\infty} \sigma^{z}\left|\sigma-e^{i \varphi}\right|^{m} \ln \left|\sigma-e^{i \varphi}\right| \frac{d \sigma}{\sigma}=\pi \frac{\Gamma(m) \Gamma(z)}{\Gamma(z+m+1)} \frac{\cos ((\pi-\varphi) z)}{\sin (\pi z)},
$$

for $-1<\mathfrak{R e}(z)<0$. Using the well-known formula

$$
\frac{\Gamma(z)}{\Gamma(z+m+1)}=\prod_{j=0}^{m} \frac{1}{(z+j)}, \quad m \in \mathbb{N},
$$

and $\Gamma(m)=(m-1)$ ! yields $(6.17)$ for $m=2 k, k \in \mathbb{N}_{0}$ :

$$
g_{k}(\varphi ; z)=(2 k) ! \prod_{j=1}^{2 k+1} \frac{\pi}{z-j} \frac{\cos ((\pi-\varphi)(z-2 k-1))}{\sin (\pi z)} .
$$

This symbol may be analytically continued. If we set

$$
h_{0}(\varphi, z):=\pi \frac{\cos ((\pi-\varphi) z)}{\sin (\pi z)},
$$

then we can write

$$
h_{k}(\varphi, z)=h_{0}(\varphi, z) \cdot(2 k) ! \prod_{j=1}^{2 k+1} \frac{\pi}{z-j} .
$$

Although the asymptotic type can be read off immediately, for the order we have two possibilities:

$$
h_{k}(\varphi ; z) \in \begin{cases}M_{P_{k}}^{-(2 k+1)} & \text { if } \vartheta=0 \text { or } \vartheta= \pm \pi, \\ M_{P_{k}}^{-\infty} & \text { if } \vartheta \in(-\pi, \pi) \backslash\{0\},\end{cases}
$$


where the Mellin asymptotic type in both cases is

$$
P_{k}=\{(j, 0) \mid j \in \mathbb{Z}\} \dot{+}\{(j, 0) \mid 1 \leq j \leq 2 k+1\} .
$$

In order to see the property $h_{k}^{\vartheta} \in M_{P_{k}}^{-\infty}$ for $\varphi \in(-\pi, \pi) \backslash\{0\}$, consider the representations $\cos (z)=\left(e^{i z}+e^{-i z}\right) / 2$ and $\sin (z)=\left(e^{i z}-e^{-i z}\right) /(2 i)$ and the limits $\tau \rightarrow \pm \infty$ for $z=\beta+i \tau, \beta \in \mathbb{R}$ fixed.

So far, we have only considered one single term in the expansions (??). In fact, we can also consider the whole series:

$$
\begin{aligned}
\tilde{h}(\lambda, z) & :=\sum_{k=0}^{\infty} \frac{(-1)^{k}}{(k !)^{2}}\left(\frac{\lambda}{2}\right)^{2 k} g_{k}(\varphi, z) \\
& =\sum_{k=0}^{\infty}(-1)^{k} \lambda^{2 k} \frac{(2 k) !}{2^{2 k}(k !)^{2}} \prod_{j=1}^{2 k+1} \frac{1}{z-j} .
\end{aligned}
$$

Notice that $\tilde{h}(\lambda, z)$ defines a meromorphic function on $\mathbb{C}$ with simple poles at $-\mathbb{N}_{0}$. Setting

$$
h(\varphi, \lambda, z):=h_{0}(\varphi, z) \tilde{h}(\lambda, z)
$$

yields the desired Mellin symbol. For $\alpha \in[0,2 \pi)$, set $h^{\alpha}(\varphi, \lambda, z):=h(\varphi-\alpha, \lambda, z)$. Using this definition, we can start to put our results together and define the potential operator's Mellin symbol at the $j^{\text {th }}$ vertex as

$$
z \mapsto \bar{h}_{\log , j}(\varphi, \lambda, z):=\left(h^{0}(\varphi, \lambda, z), h^{\alpha_{j}}(\varphi, \lambda, z)\right) \in M^{-1} \oplus M^{-1} .
$$

By the general theory of Mellin pseudodifferential operators,

$$
\mathrm{op}_{\mathrm{M}} \bar{h}_{\mathrm{log}, j}:=\left(\mathrm{op}_{\mathrm{M}} h_{j}^{0}, \mathrm{op}_{\mathrm{M}} h_{j}^{\alpha_{j}}\right): \underset{\mathcal{H}^{s, \gamma}\left(\mathbb{R}_{+}\right)}{\mathcal{H}^{s, \gamma}\left(\mathbb{R}_{+}\right)} \rightarrow \mathcal{H}^{s+\frac{1}{2}, \gamma+\frac{1}{2}}\left(X_{\alpha_{j}}^{\wedge}\right)
$$

is a continuous operator. Using this notation, we can write

$$
\Phi_{j} \mathrm{~T}_{\log }=\Phi_{j}^{\prime} \mathrm{op}_{\mathrm{M}}^{\gamma}\left[\bar{h}_{\log , j}\right] \hat{\phi}_{j}+\Phi_{j}^{\prime} \mathrm{T}_{\log }\left(1-\hat{\phi}_{j}\right) .
$$

Iterating over all $N$ vertices $v_{j}$ yields

$$
\mathrm{T}_{\log }=\sum_{j=1}^{N} \Phi_{j}^{\prime} \mathrm{op}_{\mathrm{M}}^{\gamma}\left[h_{\log , j}\right] \hat{\phi}_{j}+\left(1-\sum_{j=1}^{N} \Phi_{j}^{\prime}\right) \mathrm{T}_{\log }\left(1-\sum_{j=1}^{N} \phi_{j}\right) .
$$

Cutting out the areas around the interior of the edges yields

$$
\mathrm{T}_{\log }=\sum_{j=1}^{N} \Phi_{j}^{\prime} \mathrm{op}_{\mathrm{M}}^{\gamma}\left[\bar{h}_{\log , j}\right] \hat{\phi}_{j}+\sum_{j=1}^{N} \Theta_{j}^{\prime} \mathrm{op}_{\psi}\left[h_{\log , j}^{\psi}\right] \hat{\phi}_{j}+\mathcal{R}_{1}(\lambda)+\mathcal{R}_{2}(\lambda) .
$$

The term $\mathcal{R}_{2}$ contains terms resulting from cut-off functions which are overlapping in the interior of the edges. Naturally, these are ordinary pseudodifferential operators. Finally, set

$$
\operatorname{Sl}_{\mathrm{M}}(\lambda):=\frac{1}{2 \pi} \sum_{j=1}^{N} \Phi_{j}^{\prime} \mathrm{op}_{\mathrm{M}}^{\gamma}\left[\bar{h}_{\log , j}\right] \hat{\phi}_{j}
$$




$$
\mathrm{Sl}_{\psi}(\lambda):=\frac{1}{2 \pi} \sum_{j=1}^{N} \Theta_{j}^{\prime} \mathrm{op}_{\psi}\left[h_{j}^{\psi}\right] \theta_{j}+\mathcal{R}_{2}(\lambda) .
$$

Finally, we also have to consider the term $\mathrm{T}_{\mathrm{reg}}(\lambda)$. Setting

$$
\operatorname{Sl}_{\mathrm{G}}(\lambda):=\frac{1}{2 \pi}\left\{\mathrm{T}_{\mathrm{reg}}(\lambda)+\mathcal{R}_{1}(\lambda)\right\}
$$

we can write $\mathrm{Sl}^{\mathrm{i} / \mathrm{o}}(\lambda)=\mathrm{Sl}_{\mathrm{M}}(\lambda)+\mathrm{Sl}_{\psi}(\lambda)+\mathrm{Sl}_{\mathrm{G}}(\lambda)$, and thus have succeeded in showing that $\operatorname{Sl}(\lambda)$ is indeed a potential operator in the sense of Definition 4.4. What remains to be shown is the asymptotic behaviour of $\mathrm{Sl}^{\mathrm{i} / \mathrm{o}}(\lambda) \varphi$ in infinity, i.e. $|x| \rightarrow \infty$. But this is an easy consequence of Lemma 6.3. Hence, $\mathrm{Sl}^{\mathrm{i} / \mathrm{o}}(\lambda) \in$ $\mathcal{K}_{\mathrm{i} / \mathrm{o}}^{s+\frac{3}{2}, \gamma+\frac{3}{2}}(\Omega)$.

Corollary 6.8. There is a family of potential operators $\mathrm{T}_{\log , k} \in \mathcal{P}^{-2 k-1}(\Omega)$, $k \in \mathbb{N}_{0}$, such that

$$
\mathrm{Sl}^{\mathrm{i} / \mathrm{o}}-\frac{(-1)}{2 \pi} \sum_{k=0}^{M-1} \frac{(-1)^{k}}{(k !)^{2}}\left(\frac{\lambda}{2}\right)^{2 k} \mathrm{~T}_{\log , k} \in \mathcal{P}^{-2 M-1}(\Omega ; \Lambda)
$$

for every integer $M \geq 0$.

Theorem 6.9. The boundary limits of the operator $\mathrm{Sl}(\lambda)$ exist and are identical, i.e.

$$
\gamma_{0}^{+} \operatorname{Sl}(\lambda) f=\gamma_{0}^{-} \operatorname{Sl}(\lambda) f .
$$

Furthermore, in the sense of Definition 4.4,

$$
\gamma_{0}^{+} \operatorname{Sl}(\lambda) \in \mathcal{C}^{-1}(\partial \Omega, \mathbf{g} ; \Lambda)
$$

with $\mathbf{g}=(\gamma, \gamma+1, \Theta)$. The Mellin symbols are given by

$$
\hat{h}_{\log , j}(\lambda, z)=\left[\begin{array}{cc}
h(0, \lambda, z) & h\left(-\alpha_{j}, \lambda, z\right) \\
h\left(\alpha_{j}, \lambda, z\right) & h(0, \lambda, z)
\end{array}\right],
$$

where the functions $h(\varphi, \lambda, z)=h_{0}(\varphi, z) \tilde{h}(\lambda, z)$ are those from Theorem 6.7 .

Proof. We will first show the existence and value of the limits (3.1) for each $t \in \partial \Omega$. Only then we will recast the boundary value into a (Mellin) pseudodifferential operator in the sense of Definition 4.4.

It is clear that we will have to focus on the situation in a neighborhood of the corners $v_{j}$ since in the interior of any edge $\partial \Omega_{j}$, the behaviour is exactly the same as in the situation with smooth boundary.

$$
\mathrm{T}_{\log }=\mathrm{T}_{\log } \phi_{j}^{j+1}+\mathrm{T}_{\log }\left(1-\phi_{j}^{j+1}\right)
$$

Like in the proof of the preceding theorem, we start with some preparations. Using polar coordinates, we need to consider the limits for $r \rightarrow 0$ and $\alpha \rightarrow \alpha_{j}^{ \pm}$. In order to understand the limit $r \rightarrow 0$, consider the decomposition

$$
\mathrm{T}_{\log }=\mathrm{T}_{\log } \hat{\phi}_{j}+\mathrm{T}_{\log }\left(1-\hat{\phi}_{j}\right)
$$




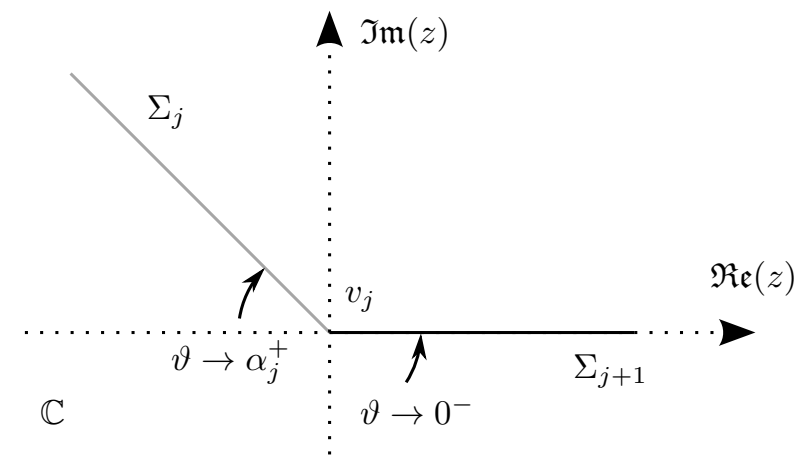

Figure 6.3: Exterior Limit

Having established the boundary limits of the single layer potential, we continue with reformulating the operator as a Mellin pseudodifferential operator. This is of course not difficult to do, since most of the work has already been done in the previous theorem.

$$
\begin{gathered}
\gamma_{0}^{ \pm} \mathrm{Sl}_{\mathrm{M}}(\lambda)=-\frac{1}{2 \pi} \sum_{j=1}^{N} t^{2 k+1} \phi_{j}^{\prime} \mathrm{op}_{\mathrm{M}}^{\gamma}\left[\hat{h}_{\mathrm{log}, j}\right] \phi_{j} \\
\gamma_{0}^{ \pm} \mathrm{Sl}_{\psi}(\lambda)=-\frac{1}{2 \pi} \sum_{j=1}^{N} \theta_{j}^{\prime} \mathrm{op}_{\psi}\left[h_{j}^{\psi}\right] \theta_{j} .
\end{gathered}
$$

The Mellin symbols for the vertices can be calculated as

$$
\hat{h}_{\log , j}(\lambda, z):=\left[\begin{array}{cc}
h(0, \lambda, z) & h\left(-\alpha_{j}, \lambda, z\right) \\
h\left(\alpha_{j}, \lambda, z\right) & h(0, \lambda, z)
\end{array}\right] .
$$

\subsection{The Double Layer Potential}

In this section we are able to reproduce the same modified jump relations as in [Kre99, equation (6.45)] by using the methods of singular analysis. We believe that these methods could lead to generalized jump relations in the three dimensional case (cf. chapter 8 ). This might be an interesting problem to tackle in future investigations.

Additionally, we can demonstrate that the double layer potential, which we have defined in equation (6.2), is not compact. To understand this, we need to consider the leading singularity in Theorem 6.5.

Theorem 6.10. The double layer potential $\mathrm{Dl}^{\mathrm{i} / \mathrm{o}}(\lambda)$ is a potential operator of order 0 . More precisely, $\mathrm{Dl}^{\mathrm{i} / \mathrm{o}} \in \mathcal{P}^{0}(\Omega ; \Lambda)$ with

$$
\mathrm{Dl}^{\mathrm{i} / \mathrm{o}}(\lambda): \mathcal{H}^{s, \gamma}(\partial \Omega) \rightarrow \mathcal{K}_{\mathrm{i} / \mathrm{o}}^{s+\frac{1}{2}, \gamma+\frac{1}{2}}(\Omega), \quad \lambda \in \Lambda_{0}^{ \pm} .
$$


More specifically, $\mathrm{Dl}(\lambda)$ may be written as the sum

$$
\mathrm{Dl}^{\mathrm{i} / \mathrm{o}}(\lambda)=\frac{1}{2 \pi}\left\{\tilde{\mathrm{T}}_{\text {sing }}+\tilde{\mathrm{T}}_{\log }(\lambda)+\tilde{\mathrm{T}}_{\mathrm{reg}}(\lambda)\right\},
$$

where $\tilde{\mathrm{T}}_{\text {sing }} \in \mathcal{P}^{0}(\Omega ; \Lambda), \tilde{\mathrm{T}}_{\log } \mathcal{P}^{-1}(\Omega ; \Lambda)$, and $\tilde{\mathrm{T}}_{\text {reg }} \in \mathcal{P}^{-\infty}(\Omega ; \Lambda)$. In particular, the operators can be written as

$$
\tilde{\mathrm{T}}_{\text {sing }}=\sum_{j=1}^{N} \Phi_{j}^{\prime} \mathrm{op}_{\mathrm{M}}\left[\bar{g}_{\text {sing }, j}\right] \phi_{j}+\sum_{j=1}^{N} \Theta_{j}^{\prime} \mathrm{op}_{\psi}\left[g_{\text {sing }, j}^{\psi}\right] \theta_{j}
$$

and

$$
\tilde{\mathrm{T}}_{\log }(\lambda)=\sum_{j=1}^{N} \Phi_{j}^{\prime} r \mathrm{op}_{\mathrm{M}}\left[\bar{g}_{\log , j}\right] \phi_{j}+\sum_{j=1}^{N} \Theta_{j}^{\prime} \mathrm{op}_{\psi}\left[g_{\log , j}^{\psi}\right] \theta_{j},
$$

where Mellin symbols are given by

$$
\begin{gathered}
\bar{g}_{\text {sing }, j}(\lambda, z)=\left(g^{\alpha_{j}}(\varphi, \lambda, z), g^{0}(\varphi, \lambda, z)\right) \in M_{P}^{0} \oplus M_{P}^{0}, \\
\bar{g}_{\log , j}(\lambda, z)=\left(g^{\alpha_{j}}(\varphi, \lambda, z), g^{0}(\varphi, \lambda, z)\right) \in M_{P}^{-1} \oplus M_{P}^{-1},
\end{gathered}
$$

where $g^{\vartheta}(\varphi, \lambda, z)=g_{0}(\varphi-\vartheta, z) \tilde{g}(\lambda, z)$ with

$$
\begin{gathered}
g_{0}(\varphi, z):=\pi \frac{\cos ((\pi-\varphi) z)}{\sin (\pi z)} \\
\tilde{g}(\lambda, z):=\sum_{k=0}^{\infty}(-1)^{k} \lambda^{2 k} \frac{(2 k) !}{2^{2 k}(k+1) !(k !)} \prod_{j=0}^{2 k} \frac{1}{z+j} .
\end{gathered}
$$

The full symbols along the edges are $h_{\mathrm{sing}, j}^{\psi} \in S^{0}(I \times I \times \mathbb{R})$ and $h_{\mathrm{log}, j} \in S_{\mathrm{cl}}^{-1}(I \times$ $I \times \mathbb{R})$.

Proof. The proof follows essentially the same line of thought as the one for Theorem 6.7: The continuity of $\mathrm{Dl}^{\mathrm{i} / \mathrm{o}}(\lambda)$ is established in the same manner as for the single layer potential.

Next, for the calculation of the Mellin symbols at the vertices, we first consider the term $\tilde{\mathrm{T}}_{\text {sing. }}$. We start with realization that

$$
\tilde{\mathrm{T}}_{\text {sing }} \psi(x):=\sum_{j=1}^{N} \int_{\partial \Omega_{j}} \tilde{t}_{\text {sing }}(x-y) \psi_{j}(y) d s(y),
$$

with the kernel

$$
\tilde{t}_{\text {sing }}(x-y)=\frac{\nu(y)(x-y)}{|x-y|^{2}} .
$$

We perform an affine transformation such that we can locally identify $\Gamma_{j+1} \cong$ $\mathbb{R}_{+}$. The nominator can then be written as

$$
\left.\nu(y)(x-y)\right|_{y_{2}=0}=x_{2} .
$$

Using polar coordinates, in particular setting $x_{2}=r \sin \vartheta$, we gain for the operator kernel:

$$
\tilde{t}_{\text {sing }}(r, \varphi, s)=-\frac{r \sin \varphi}{\left|r e^{i \varphi}-s\right|^{2}}=-\frac{1}{s^{2}} \frac{r \sin \varphi}{\left|e^{-i \varphi}-\frac{r}{s}\right|^{2}} .
$$


The operator may therefore be written as

$$
\tilde{\mathrm{T}}_{\text {sing }} \phi_{j}^{j+1} \psi_{j+1}(r, \varphi)=\int_{0}^{\infty}-\frac{r}{s} \frac{\sin \varphi}{\left|e^{-i \varphi}-\frac{r}{s}\right|^{2}} \phi_{j}^{j+1} \psi_{j+1}(s) \frac{d s}{s} .
$$

Rewriting the kernel's denominator as

$$
\begin{aligned}
\left|e^{-i \varphi}-\sigma\right|^{2} & =1-2 \sigma \cos \varphi+\sigma^{2} \\
& =1+2 \sigma \cos (\pi-\varphi)+\sigma^{2},
\end{aligned}
$$

and using [GR07, formula $(\mathbf{3 . 2 5 2}, 12)]$, we may calculate:

$$
\frac{1}{2} \int_{0}^{\infty} \sigma^{z}\left(\frac{-\sigma \sin \varphi}{\left|e^{-i \varphi}-\sigma\right|^{2}}\right) \frac{d \sigma}{\sigma}=\pi \frac{\sin ((\pi-\varphi) z)}{\sin (\pi z)} .
$$

This identity is true for $-1<\mathfrak{R e}(z)<1$ and $0<\varphi<2 \pi$, but can be analytically continued. With $0<\varphi<\alpha_{j}$ this means that

$$
\tilde{\mathrm{T}}_{\text {sing }} \phi_{j}^{j} \psi_{j}(r)=\int_{0}^{\infty} \int_{\Gamma_{\frac{1}{2}}}\left(\frac{r}{s}\right)^{-z} \tilde{g}_{\text {sing }}(z ; \varphi) \psi_{j}(s) d z \frac{d s}{s}
$$

with

$$
\tilde{g}_{\text {sing }}(z ; \varphi)=\pi \frac{\sin ((\pi-\varphi) z)}{\sin (\pi z)}, \quad-1<\mathfrak{R e}(z)<1 .
$$

As in the proof of Theorem 6.7, this can be interpreted as a Mellin symbol $M_{Q}^{0}\left(X_{\alpha_{j}}\right)$. Furthermore, the corresponding operator does not induce any shift of the conormal order. The contribution from the adjoint edge, $\tilde{T}_{\text {sing }} \phi_{j}^{j}$, is

$$
\tilde{\mathrm{T}}_{\text {sing }} \phi_{j}^{j} \psi(r, \varphi)=\int_{0}^{\infty}-\frac{r}{s} \frac{\sin \left(\varphi-\alpha_{j}\right)}{\left|e^{-i\left(\varphi-\alpha_{j}\right)}-\frac{r}{s}\right|^{2}} \phi_{j}^{j} \psi_{j}(s) \frac{d s}{s} .
$$

The Mellin symbol at the $j^{\text {th }}$ vertex is therefore given by

$$
\tilde{g}_{\text {sing }}=\left(\tilde{g}_{j}^{\alpha}(\lambda, \varphi, z), \tilde{g}^{0}(\lambda, \varphi, z)\right),
$$

and therefore

$$
\tilde{\mathrm{T}}_{\text {sing }}=\mathrm{op}_{\mathrm{M}}^{\gamma}\left[\bar{g}_{\text {sing }, j}\right] \hat{\phi}_{j}+\tilde{\mathrm{T}}_{\log }\left(1-\hat{\phi}_{j}\right) .
$$

Calculating the symbols of the second term, $\tilde{T}_{\text {log }}$ is done in the same manner as in the case of the single layer potential. Again, we are able to sum everything up, with the result

$$
\tilde{g}(\lambda, \varphi, z):=\sum_{k=0}^{\infty} \frac{(-1)^{k}}{(k+1) ! k !}\left(\frac{\lambda}{2}\right)^{2 k} \prod_{j=0}^{2 k} \frac{1}{z+j} .
$$

Iterating over all $N$ vertices $v_{j}$ yields:

$$
\tilde{\mathrm{T}}_{\log }(\lambda)=\sum_{j=1}^{N} \operatorname{op}_{\mathrm{M}}^{\gamma}\left[\bar{g}_{\log , j}\right] \hat{\phi}_{j}+\tilde{\mathrm{T}}_{\log }(\lambda)\left(1-\sum_{j=1}^{N} \hat{\phi}_{j}\right) .
$$


Taking the interior of the curvilinear edges into account:

$$
\tilde{\mathrm{T}}_{\log }=\sum_{j=1}^{N} \Phi_{j} \mathrm{op}_{\mathrm{M}}^{\gamma}\left[g_{\log , j}\right] \hat{\phi}_{j}+\sum_{j=1}^{N} \Theta_{j} \mathrm{op}_{\psi}\left[g_{j}^{\psi}\right] \theta_{j}+\mathcal{R}
$$

where

$$
\mathcal{R}=\left(1-\sum_{j=1}^{N}\left(\Phi_{j}+\Theta_{j}\right)\right) \tilde{\mathrm{T}}_{\log }
$$

We can reaggange the terms and finally set

$$
\begin{aligned}
\mathrm{Dl}_{\mathrm{M}}^{\mathrm{i} / \mathrm{o}}(\lambda) & :=\sum_{j=1}^{N} \Phi_{j} \mathrm{op}_{\mathrm{M}}^{\gamma}\left[\bar{g}_{\text {sing }, j}\right] \phi_{j}+\sum_{j=1}^{N} \Phi_{j} \mathrm{op}_{\mathrm{M}}^{\gamma}\left[\bar{g}_{\log , j}\right] \phi_{j}, \\
\mathrm{Dl}_{\psi}^{\mathrm{i} / \mathrm{o}}(\lambda) & :=\sum_{j=1}^{N} \Theta_{j} \mathrm{op}_{\psi}\left[g_{\mathrm{sing}, j}^{\psi}\right] \theta_{j}+\sum_{j=1}^{N} \Theta_{j} \mathrm{op}_{\psi}\left[g_{\mathrm{log}, j}^{\psi}\right] \theta_{j} .
\end{aligned}
$$

Absorbing the mollifying terms into $\mathrm{Dl}_{\mathbb{G}}^{\mathrm{i} / \mathrm{o}}(\lambda)$, we have achieved the decomposition in the sense of Definition 4.4.

Again, it remains to demonstrate the asymptotic behaviour for $|x| \rightarrow \infty$. But this is again only an application of lemma . Therefore, $\mathrm{Dl}^{\mathrm{i} / \mathrm{o}}(\lambda) f \in \mathcal{K}_{\mathrm{i} / \mathrm{o}}^{s+\frac{1}{2}, \gamma+\frac{1}{2}}(\Omega)$, as claimed.

Corollary 6.11. There exists a family of potential operators $\tilde{\mathrm{T}}_{\log , k} \in \mathcal{P}^{-2 k}(\Omega)$, $k \in \mathbb{N}_{0}$, such that

$$
\mathrm{Dl}^{\mathrm{i} / \mathrm{o}}-\frac{1}{2 \pi} \tilde{\mathrm{T}}_{\text {sing }}-\frac{(-1)}{2 \pi} \sum_{k=0}^{M-1} \frac{(-1)^{k}}{(k !)^{2}}\left(\frac{\lambda}{2}\right)^{2 k} \tilde{\mathrm{T}}_{\log , k} \in \mathcal{P}^{-2 M}(\Omega, \Lambda)
$$

for every integer $M \geq 0$.

Definition 6.12. For $\psi \in \prod_{j=1}^{N} C_{c}^{\infty}\left(\partial \Omega_{j}\right), \lambda \in \Lambda$, and $x \in \partial \Omega$ define the operator

$$
\mathrm{N}(\lambda) \psi(x):=\sum_{j=1}^{N} \int_{\partial \Omega_{j}} \frac{\partial}{\partial \nu_{j}(y)}\left(r_{0}^{\mathrm{i} / \mathrm{o}}(\lambda|x-y|)\right) \psi_{j}(y) d s_{j}(y) .
$$

Note that this operator is already defined on the boundary, in contrast to the definition of the double layer potential.

Theorem 6.13. The exterior and interior limits of $\mathrm{Dl}^{\mathrm{i} / \mathrm{o}}(\lambda)$ satisfy certain jump conditions. For $\psi \in H^{s}(\partial \Omega), s>1 / 2$, i.e. for conventional Sobolev spaces, they are given by

$$
\gamma_{0}^{ \pm} \operatorname{Dl}(\lambda) \psi(x)= \begin{cases} \pm \frac{1}{2} \psi(x)-\mathrm{N}(\lambda) \psi(x) & \text { for } x \in \cup_{j=1}^{N} \partial \Omega_{j} \\ \pm \frac{1}{2} \delta_{j}^{ \pm} \psi(x)-\mathrm{N}(\lambda) \psi(x) & \text { for } x \in\left\{v_{j} \mid j=1, \ldots, N\right\}\end{cases}
$$

where

$$
\delta_{j}^{+}=\frac{1}{\pi}\left(2 \pi-\alpha_{j}\right), \quad \text { and } \quad \delta_{j}^{-}=\frac{\alpha_{j}}{\pi} .
$$


Both limits, denoted by $C_{00}^{ \pm}(\lambda) \psi=\gamma_{0}^{ \pm} \mathrm{Dl}(\lambda) \psi$ are elements of the algebra $\mathcal{C}^{0}(\partial \Omega, \mathbf{g} ; \Lambda)$, where $\mathbf{g}=(\gamma, \gamma, \Theta)$.

The operator $\mathrm{N}(\lambda)$ can be represented, modulo a smoothing term, as

$$
\mathrm{C}_{00}^{ \pm}(\lambda)=\sum_{j=1}^{N} \hat{\phi}_{j}^{\prime} \mathrm{op}_{\mathrm{M}}\left[\hat{g}_{\text {sing }, j}\right] \hat{\phi}_{j}+\sum_{j=1}^{N} \hat{\phi}_{j}^{\prime} \mathrm{op}_{\mathrm{M}}\left[\hat{g}_{\text {sing }, j}\right] \hat{\phi}_{j}+\sum_{j=1}^{N} \hat{\theta}_{j}^{\prime} \mathrm{op}_{\mathrm{M}}\left[g_{j}^{\psi}\right] \hat{\theta}_{j}
$$

The Mellin symbols of the resulting operators at the vertices $v_{j}$ are given by

$$
\hat{g}_{\text {sing }, j}(\lambda, z):=\left[\begin{array}{cc}
\tilde{g}_{\text {sing }}(0, z) & \tilde{g}_{\text {sing }}\left(-\alpha_{j}, z\right) \\
\tilde{g}_{\text {sing }}\left(\alpha_{j}, z\right) & \tilde{g}_{\text {sing }}(0, z)
\end{array}\right]
$$

and

$$
\hat{g}_{\log , j}(\lambda, z):=\left[\begin{array}{cc}
g_{\log }(0, \lambda, z) & g_{\log }\left(-\alpha_{j}, \lambda, z\right) \\
g_{\log }\left(\alpha_{j}, \lambda, z\right) & g_{\log }(0, \lambda, z)
\end{array}\right],
$$

where the functions $g(\varphi, \lambda, z)$ are given by

$$
\tilde{g}_{\text {sing }}(\varphi, z)=\pi \frac{\sin ((\pi-\varphi) z)}{\sin (\pi z)}
$$

and

$$
g_{\log }(\varphi, \lambda, z)=h_{0}(\varphi, z) \cdot \sum_{k=0}^{\infty}(-1)^{k} \frac{(2 k) !}{(k+1) ! k !}\left(\frac{\lambda}{2}\right)^{2 k} \prod_{j=1}^{2 k+1} \frac{1}{z-j},
$$

respectively.

Proof. The method of proof is exactly the same as in Theorem 6.9. However, the operator $\tilde{\mathrm{T}}_{\text {sing }}$ will yield more interesting boundary limits. As in Theorem 6.9 we consider the decomposition

$$
\tilde{\mathrm{T}}_{\text {ing }}=\tilde{\mathrm{T}}_{\text {sing }} \phi_{j}^{j+1}+\tilde{\mathrm{T}}_{\text {sing }}\left(1-\phi_{j}^{j+1}\right) .
$$

Again, we primarily need to consider the first two terms. The first term, using Cartesian coordinates such that $\partial \Omega_{j+1}$ is identified with the positive half-line of real numbers. Taking advantage of complex polar coordinates, we can write this operator's kernel as

$$
\tilde{\mathrm{T}}_{\text {sing }} \phi_{j}^{j+1} \psi(r, \varphi)=\int_{0}^{\infty}-\frac{r}{s} \frac{\sin \varphi}{\left|e^{-i \varphi}-\frac{r}{s}\right|^{2}} \phi_{j}^{j+1} \psi_{j+1}(s) \frac{d s}{s}
$$

We are interested in the boundary limits, where $t \in \partial \Omega_{j}$ and $\phi_{j}^{j+1}(t)=1$. We can show the limit either directly in 'x-space' or by using the Mellin operator formulation. We start with the former: Consider the decomposition $\left|e^{i \varphi}-\sigma\right|^{2}=$ $\left(e^{i \varphi}+\sigma\right)\left(e^{-i \varphi}-\sigma\right)$ of the denominator in (6.40):

$$
\frac{-\sigma \sin \varphi}{\left(e^{i \varphi}+\sigma\right)\left(e^{-i \varphi}-\sigma\right)}=\frac{1}{2 i}\left(\frac{e^{i \varphi}}{e^{i \varphi}-\sigma}-\frac{e^{-i \varphi}}{e^{-i \varphi}-\sigma}\right) .
$$

One can easily see that, using the well-known distributional identity

$$
\lim _{\epsilon \rightarrow 0^{+}} \frac{1}{x \pm i \epsilon}=\mp i \pi \delta(x)+\mathrm{PV} \frac{1}{x},
$$


PV being the principal values, that

$$
\lim _{\varphi \rightarrow 0^{+}} \frac{e^{ \pm i \varphi}}{e^{ \pm i \varphi}-\sigma}= \pm i \pi \delta(\sigma-1)-\mathrm{PV} \frac{1}{\sigma-1}
$$

Notice the different sign on the right-hand side above. For the limit from the exterior, i.e. $\vartheta \rightarrow 0^{-}$, we obtain

$$
\lim _{\varphi \rightarrow 0^{-}} \frac{e^{ \pm i \varphi}}{e^{ \pm i \varphi}-\sigma}=\mp i \pi \delta(\sigma-1)+\mathrm{PV} \frac{1}{\sigma-1}
$$

As a result,

$$
\lim _{\varphi \rightarrow 0^{ \pm}} \frac{-\sigma \sin \varphi}{\left|e^{i \varphi}-\sigma\right|^{2}}= \pm \pi \delta(\sigma-1)
$$

in the sense of distributions, or

$$
\lim _{\varphi \rightarrow 0^{ \pm}} \int_{0}^{\infty} \frac{-\sigma \sin \varphi}{\left|e^{i \varphi}-\sigma\right|^{2}} \phi_{j}^{j+1} \psi_{j+1}(s) d s= \pm \frac{1}{2} \psi_{j+1}(t) .
$$

The limit in terms of the Mellin operator formulation is simply by the observation

$$
\lim _{\varphi \rightarrow 0} \int_{\Gamma_{\frac{1}{2}}} r^{-z} \frac{\sin ((\pi-\varphi) z)}{\sin (\pi z)} \mathcal{M}\left(\psi_{j+1}\right)(z) d z
$$

But in this case, the limit can simply be pulled under the integral which yields

$$
\frac{1}{2} \int_{\Gamma_{\frac{1}{2}}} r^{-z} \mathcal{M} f(z) d z= \pm \frac{1}{2} \psi_{j+1}(r)
$$

The second term is given by

$$
\tilde{\mathrm{T}}_{\text {sing }}\left(1-\phi_{j}^{j+1}\right) \psi(x)=\sum_{j=1}^{N} \int_{\partial \Omega_{j}} t_{\log }(|x-y| ; \lambda)\left(1-\phi_{j}^{j+1}\right) \psi_{j}(y) d s(y) .
$$

But $x \neq y$, which means that $\tilde{\mathrm{T}}_{\log }\left(1-\phi_{j}^{j+1}\right) \psi \in C^{\infty}$. Therefore, the limit exists anyway and is identical to

$$
\tilde{\mathrm{T}}_{\text {sing }}\left(1-\phi_{j}^{j+1}\right) \psi(t)=\sum_{j=1}^{N} \int_{\partial \Omega_{j}} t_{\log }(|t-y| ; \lambda)\left(1-\phi_{j}^{j+1}\right) \psi(y) d s(y) .
$$

Also notice that

$$
\int_{\partial \Omega_{j}} t_{\operatorname{sing}}(|t-y| ; \lambda) \phi_{j}^{j+1} \psi_{j}(y) d s(y)=0 .
$$

As a consequence,

$$
\gamma^{ \pm} \operatorname{Sl}(\lambda) \psi(t)= \pm \psi(t)-\mathrm{N}(\lambda) \psi(t) \quad \text { for } t \in[0, \epsilon) .
$$

The argument is the same for all $t \in \partial \Omega_{j}$ such that $\phi_{j}^{j}(t)=1$. In order to obtain the limit for $\Omega \ni x \rightarrow v_{j}$ or $\Omega^{\mathrm{c}} \ni x \rightarrow v_{j}$, i.e. the limit for a corner point 
either from the inside or outside of the obstacle, we have to apply a standard technique of singular analysis. Consider the decomposition

$$
\tilde{\mathrm{T}}_{\log }=\tilde{\mathrm{T}}_{\log } \hat{\phi}_{j}+\tilde{\mathrm{T}}_{\log }\left(1-\hat{\phi}_{j}\right) .
$$

So far, we have assumed that $\psi_{j} \in \mathcal{H}^{s, \gamma}\left(\partial \Omega_{j}\right), j=1, \ldots, N$. Assume instead $\psi \in H^{s}(\partial \Omega)$, where the restrictions $\psi_{j}=\left.\psi\right|_{\partial \Omega_{j}}$ fulfil the compatibility conditions (3.9) at both ends of each segment of the boundary.

The first two terms in (6.41) will be interpreted as Mellin pseudodifferential operators, just as we have derived in Theorem 6.10 . This means that we write

$$
\tilde{\mathrm{T}}_{\log } \hat{\phi}_{j}=\Phi_{j}^{\prime} \mathrm{op}_{\mathrm{M}}^{\gamma} \hat{\phi}_{j}+\mathcal{R}(\lambda),
$$

where $\mathcal{R}$ is a regularizing term which vanishes in the vicinity of the vertex:

$$
\mathcal{R}=\left(1-\Phi^{\prime}\right) \tilde{\mathrm{T}}_{\log } \hat{\phi}_{j}
$$

with $\Phi^{\prime}:=\sum_{j=1}^{N} \Phi_{j}^{\prime}$. Since $\phi_{j}^{j+1} \psi_{j+1} \in H^{s}\left(\partial \Omega_{j+1}\right)$, its Mellin transform has simple poles at $0,-1, \ldots,-\kappa(s)$. In particular,

$$
\mathcal{M}\left(\phi_{j}^{j+1} \psi_{j+1}\right)(z)-\frac{\psi_{j+1}\left(v_{j}\right)}{z} \in \mathcal{A}(\{-1<\mathfrak{R e}(z)<1\}),
$$

i.e. the difference is a holomorphic function on the strip $\{z \in \mathbb{C} \mid-1<\mathfrak{R e}(z)<$ 1 . Observe that the Mellin symbol (6.30) has at most simple poles at $\mathbb{Z} \backslash\{0\}$. From this we can conclude that

$$
\operatorname{res}_{z=0}\left(\pi \frac{\sin ((\pi-\varphi) z)}{\sin (\pi z)} \mathcal{M}\left(\phi_{j}^{j+1} \psi_{j+1}\right)(z)\right)=(\pi-\varphi) \psi_{j+1}\left(v_{j}\right) .
$$

As a result, for $1 / 2<\beta<1$, we have

$$
\int_{0}^{\infty} \int_{\Gamma_{\frac{1}{2}-\beta}}\left(\frac{r}{s}\right)^{-z} \tilde{g}_{\mathrm{sing}, j}^{0}(z ; \varphi) \psi_{j}(s) d z \frac{d s}{s}-(\pi-\varphi) \psi_{j}\left(v_{j}\right) \in r^{\beta} \mathcal{H}^{\infty}\left(X_{\alpha_{j}}^{\wedge}\right) .
$$

Analogously, for the second term:

$\int_{0}^{\infty} \int_{\Gamma_{\frac{1}{2}-\beta}}\left(\frac{r}{s}\right)^{-z} \tilde{g}_{\mathrm{sing}, j}^{\alpha_{j}}(z ; \varphi) \psi_{j}(s) d z \frac{d s}{s}+\left(\pi-\left(\varphi-\alpha_{j}\right)\right) \psi_{j}\left(v_{j}\right) \in r^{\beta} \mathcal{H}^{\infty}\left(X_{\alpha_{j}}^{\wedge}\right)$.

We have to be careful with the signs. For example, the orientation of normal vector field matters. Moreover, we have to remind ourselves of the compatibility conditions, which imply $\psi_{j}\left(v_{j}\right)=\psi_{j+1}\left(v_{j}\right)$. Adding these two terms leads us to the limit $\delta^{-} \psi\left(v_{j}\right)$ as desired:

$$
\frac{1}{2 \pi}\left(\mathrm{op}_{\mathrm{M}}\left[\bar{g}_{\mathrm{sing}, j}\right] \hat{\phi}_{j} \psi\right)-\left(-\frac{\alpha_{j}}{\pi}\right) \psi_{j}\left(v_{j}\right) \in r^{\beta} \mathcal{H}^{\infty}\left(X_{\alpha_{j}}^{\wedge}\right) .
$$

For the limit $r \rightarrow 0$ from the exterior, we can proceed analogously by rotating the coordinate system by the angle $\alpha_{j}$, changing signs for the Mellin symbols due to the relative orientation of the normal vector fields, and using the angle $2 \pi-\alpha_{j}$ instead of $\alpha_{j}$. The corresponding asymptotics are

$$
\frac{1}{2 \pi}\left(\mathrm{op}_{\mathrm{M}}\left[\bar{g}_{\mathrm{sing}, j}\right] \hat{\phi}_{j} \psi\right)-\left(\frac{2 \pi-\alpha_{j}}{\pi}\right) \psi_{j}\left(v_{j}\right) \in r^{\beta} \mathcal{H}^{\infty}\left(X_{2 \pi-\alpha_{j}}^{\wedge}\right) .
$$


In both cases, $\beta>1 / 2$, so the remainder functions which are (locally) in $r^{\beta} \mathcal{H}^{\infty}\left(X_{\theta}^{\wedge}\right), \theta \in\left\{\alpha_{j}, 2 \pi-\alpha_{j}\right\}$, both vanish as $r \rightarrow 0$.

$$
\gamma_{0}^{ \pm} \mathrm{Dl}_{\mathrm{M}}(\lambda)=\frac{1}{2 \pi} \sum_{j=1}^{N} t^{2 k} \hat{\phi}_{j}^{\prime} \mathrm{op}_{\mathrm{M}}^{\gamma}\left[\hat{g}_{\log , j}\right] \phi_{j}
$$

The calculation of the Mellin symbols is done in a straightforward way:

$$
\hat{g}_{\log , j}(\lambda, z):=\left[\begin{array}{cc}
g(0, \lambda, z) & g\left(-\alpha_{j}, \lambda, z\right) \\
g\left(\alpha_{j}, \lambda, z\right) & g(0, \lambda, z)
\end{array}\right] .
$$

The pseudodifferential symbols in the interior of the edges are again calculated in the familiar fashion:

$$
\gamma_{0}^{ \pm} \mathrm{Dl}_{\psi}(\lambda)=\frac{1}{2 \pi} \sum_{j=1}^{N} \theta_{j}^{\prime} \mathrm{op}_{\psi}\left[g_{j}^{\psi}\right] \theta_{j},
$$

where $g_{j}^{\psi} \in S^{0}\left(I_{j} \times I_{j} \times \mathbb{R}\right)$. 


\section{Chapter 7}

\section{Construction of the Parametrix and Proof of the Main Theorem}

As the title of this penultimate chapter suggests, we will perform and apply a parametrix construction for the boundary value of the single layer potential, $\mathrm{C}_{01}$. However, one observes that the singular nature of the polygonal boundary comes into play. One important observation is the appearence of the Green operators. The original proof of Melrose [Mel84] utilizes standard pseudodifferential calculus for smooth manifolds. Due to the presence of the singularities in the corner however, an alternative approach has to be pursued. Instead of using the standard Laplacian, we will construct a parametrix as an element of the algebra $\mathcal{C}^{1}(\partial \Omega, \mathbf{g})$.

\subsection{Ellipticity and Parametrix Construction}

Theorem 7.1. The operator $\mathrm{C}_{01}(\lambda)$ is elliptic. In particular, there exists a parametrix $\mathrm{Q} \in \mathcal{C}^{1}(\partial \Omega, \mathbf{g}), \mathbf{g}=(1, \gamma, \Theta)$, such that

$$
\mathrm{QC}_{01}(\lambda)=1+\mathscr{R}(\lambda),
$$

where $\mathscr{R}(\lambda): H^{s}(\partial \Omega) \rightarrow H^{s+2}(\partial \Omega)$ is an entire family of compact operators.

Remark 7.2. Notice that with regards to Boutet de Monvel's algebra, the parametrix corresponds to an operator of the form

$$
\mathcal{A}_{\text {para }}=\left[\begin{array}{ll}
0 & 0 \\
0 & \mathrm{Q}
\end{array}\right] \in \mathcal{B}^{1}(\Omega) .
$$

Proof. Recall that the Mellin symbol at the vertex $v_{j}$ for the operator $C_{01}(\lambda)$ is given by the product $\hat{h}_{j}(\varphi, \lambda, z)=\hat{h}_{j, 0}(\varphi, z) \tilde{h}(\lambda, z)$, where the first factor is given by

$$
\hat{h}_{j, 0}(z)=\pi\left[\begin{array}{cc}
\cot \pi(z-1) & \frac{\cos \left(\left(\pi+\alpha_{j}\right)(z-1)\right)}{\sin (\pi z)} \\
\frac{\cos \left(\left(\pi-\alpha_{j}\right)(z-1)\right)}{\sin (\pi z)} & \cot \pi(z-1),
\end{array}\right] .
$$


Notice that the off-diagonal functions are of class $M_{P}^{-\infty}$. The second factor can be expanded as

$$
\tilde{h}(\lambda, z)=\frac{1}{z+1} \frac{1}{z} \cdot\left[1+\sum_{k=1}^{\infty}(-1)^{k} \lambda^{2 k} \frac{(2 k) !}{2^{2 k}(k !)^{2}} \prod_{j=2}^{2 k} \frac{1}{z+j}\right] \cdot \mathbb{1}_{2 \times 2} .
$$

In order to construct the parametrix, we therefore focus on inverting the factor $h_{0}$. We start with calculating the determinant,

$$
\operatorname{det} h_{j, 0}(z)=\pi^{2} \frac{\sin ^{2}\left(\alpha_{j} z\right)}{\sin ^{2}(\pi z)},
$$

and conclude that $h_{j, 0}$ is invertible as long as $\Gamma_{\gamma}$ for the weight $\gamma$ does not cross the poles or roots of the determinant. The potential locations for those are easy to determine:

$$
z_{\text {inv }} \in \mathbb{Z} \quad \text { or } \quad z_{\text {inv }} \in \frac{\pi}{\alpha_{j}} \mathbb{Z} .
$$

Notice that nominator and denominator in (7.3) can cancel each other out for $z \neq 0$. This is, as will see in the example in section 7.2, the case for $\alpha_{j}=\pi / 2$ and $z \in 2 \mathbb{Z}$. Calculating the inverse matrix yields:

$$
\hat{h}_{j, 0}(z)^{-1}=\frac{1}{\pi} \frac{\sin ^{2}(\pi z)}{\sin ^{2}\left(\alpha_{j} z\right)}\left[\begin{array}{cc}
\cot (\pi z) & -\frac{\cos \left(\left(\pi+\alpha_{j}\right) z\right)}{\sin (\pi z)} \\
-\frac{\cos \left(\left(\pi-\alpha_{j}\right) z\right)}{\sin (\pi z)} & \cot (\pi z)
\end{array}\right] .
$$

Note that the Mellin asymptotic type for the inverse matrix is a subset of

$$
R_{j} \subseteq(\mathbb{Z} \times\{1\}) \dot{+}\left\{\left(\frac{\pi}{\alpha_{j}} j, 1\right) \mid j \in \mathbb{Z} \backslash\{0\}\right\} .
$$

Set $\hat{q}_{j}(z):=\hat{h}_{j, 0}^{-1}(z)$ and define the parametrix by

$$
\mathrm{Q}:=\sum_{j=1}^{N} t^{-1} \hat{\phi}_{j}^{\prime} \mathrm{op}_{\mathrm{M}}^{\gamma}\left[\hat{q}_{j}\right] \hat{\phi}_{j}+\sum_{j=1}^{N} \theta_{j}^{\prime} \mathrm{op}_{\psi}\left[q_{j}\right] \theta_{j}
$$

By the standard theory of Mellin pseudodifferential operators, the parametrix is a continuous mapping

$$
\mathrm{Q}: \mathcal{H}^{s, \gamma}(\partial \Omega) \rightarrow \mathcal{H}^{s-1, \gamma-1}(\partial \Omega),
$$

with norm $\|Q\| \leq \sum_{j=1}^{N} \sum_{m, n=1,2} c\left(q_{i}^{m, n}\right)$, where

$$
c\left(q_{j}\right):=\sup _{z \in \Gamma_{\beta}}\left\|\langle\mathfrak{I m}(z)\rangle^{s-1} q_{j}(z)\langle\mathfrak{I m}(z)\rangle^{-s}\right\|_{\mathcal{L}\left(L^{2}\right)}<\infty .
$$

Furthermore, it induces asymptotics

$$
\mathrm{Q}: \mathcal{H}^{s, \gamma}(\partial \Omega) \rightarrow \mathcal{H}_{R_{j}}^{s-1, \gamma-1}(\partial \Omega) .
$$

The application of the parametrix is essentially given by Theorem 4.10. Since we have not given a proof of that theorem, we outline the technicalities here. In order to technically simplify the proof, we assume that the edges $\partial \Omega_{j}$ of the boundary consist of straight lines. Edges which are smoothly curved away from 
the vertices, are covered by the standard theory of pseudodifferential calculus. Write

$$
\mathrm{T}_{\log }=\sum_{j=1}^{N} \hat{\phi}_{j}^{\prime} \mathrm{op}_{\mathrm{M}}\left[\hat{h}_{\log , j}\right] \hat{\phi}_{j},
$$

where $\hat{h}_{\log , j}, j=1, \ldots, N$, is the family of $2 \times 2$-matrix valued Mellin symbols. Application of the parametrix yields, using $\hat{\phi}_{j}^{\prime} \hat{\phi}_{j}=\hat{\phi}_{j}$,

$$
\mathrm{QT}_{\log }=\sum_{j=1}^{N} \hat{\phi}_{j}^{\prime} \mathrm{op}_{\mathrm{M}}\left[\hat{q}_{j}\right] \hat{\phi}_{j} \mathrm{op}_{\mathrm{M}}\left[\hat{h}_{\log , j}\right] \hat{\phi}_{j} .
$$

Using $\hat{\phi}_{j}=1-\left(1-\hat{\phi}_{j}\right)$, where 1 is the identity on $\mathcal{H}^{s, \gamma}(\partial \Omega)$, and Lemma 4.6 ,

$$
\mathrm{QT}_{\log }=\sum_{j=1}^{N} \hat{\phi}_{j}^{\prime} \mathrm{op}_{\mathrm{M}}\left[\hat{q}_{j} \hat{h}_{\log , j}\right] \hat{\phi}_{j}-\underbrace{\sum_{j=1}^{N} \hat{\phi}_{j}^{\prime} \mathrm{op}_{\mathrm{M}}\left[\hat{g}_{j}\right]\left(1-\hat{\phi}_{j}\right) \mathrm{op}_{\mathrm{M}}\left[\hat{h}_{j}\right] \hat{\phi}_{j}}_{\mathrm{S}:=} .
$$

But $\hat{q}_{j} \hat{h}_{\log , j}=\tilde{h} \cdot \mathbb{1}_{2 \times 2}$ for $j=1, \ldots, N$. Finally, we set

$$
\mathscr{R}(\lambda):=\sum_{j=1}^{N} \hat{\phi}_{j}^{\prime} \mathrm{op}_{\mathrm{M}}[\tilde{h}(\lambda)] \hat{\phi}_{j}+\mathrm{S}
$$

with

$$
\tilde{h}(\lambda, z)=\frac{1}{z+1} \frac{1}{z} \cdot\left[\sum_{k=1}^{\infty}(-1)^{k} \lambda^{2 k} \frac{(2 k) !}{2^{2 k}(k !)^{2}} \prod_{j=2}^{2 k} \frac{1}{z+j}\right] \cdot \mathbb{1}_{2 \times 2} .
$$

One can easily see that $h^{\prime}(\lambda, z)$ converges and is holomorphic in the half-plane $\{z \mid-2<\mathfrak{R e}(z)\}$. As a result, the Mellin asymptotic type of $\tilde{h}$ induces asymptotics such that

$$
\mathscr{R}(\lambda): H^{s}(\partial \Omega) \rightarrow H^{s+2}(\partial \Omega) .
$$

As a preparation for the proof of the main theorem in chapter ??, we will need a specific estimate for the composition $\mathrm{QT}_{\log , k}, k \in \mathbb{N}_{0}$ :

Proposition 7.3. For each $k \in \mathbb{N}_{0}$ :

$$
\left\|\mathrm{QT}_{\log , k}\right\|_{s, \gamma, s+2 k, \gamma+2 k} \leq(2 k !) .
$$

Proof.

$$
\mathrm{QT}_{\log , k}: \mathcal{H}^{s, \gamma}(\partial \Omega) \rightarrow \mathcal{H}^{s+2 k, \gamma+2 k}(\partial \Omega),
$$

We know by standard theory that $\mathrm{T}_{\log , k}$ is a continuous operator $\mathcal{H}^{s, \gamma}(\partial \Omega) \rightarrow$ $\mathcal{H}^{s+2 k+1, \gamma+2 k+1}(\partial \Omega)$. Let $s \in \mathbb{N}_{0}, \gamma \geq 0$ real numbers. For $g \in \mathcal{H}^{s, \gamma}\left(\Omega^{c}\right)$, consider the integral operator

$$
F g(x):=\int_{\Omega^{c}} \ln (|x-y|)|x-y|^{2 k} g(y) d y, \quad x \in \Omega^{c},
$$


which defined a function in the interior of the obstacle, $F g \in \mathcal{H}^{s+2 k+\frac{3}{2}, \gamma+2 k+\frac{3}{2}}\left(\Omega^{\mathrm{c}}\right)$.

$$
\|F g\|_{m, \gamma}^{2}=\sum_{|\alpha| \leq m} \int_{\Omega^{c}} r^{2(|\alpha|-\gamma-m)}\left|D^{\alpha} F g(x)\right|^{2} d x,
$$

with $r=|x|, m \in \mathbb{N}_{0}$, and differentiating yields the norm $\|F g\|_{m+2 k+1, \gamma} \leq$ $(2 k) !\|g\|_{m, \gamma}$. Using complex interpolation, we can extend the result to

$$
\|F g\|_{m+2 k+\frac{3}{2}, \gamma} \leq c(2 k) !\|g\|_{m, \gamma}, \quad c>0 .
$$

The factorial can be found by using the identity

$$
\frac{d^{n}}{d x^{n}}\left(\ln (|x|) x^{2 k}\right)=x^{2 k-n}(2 k)_{n}(\ln (|x|)+\psi(2 k+1)+\psi(2 k+1-n)),
$$

where $(k)_{n}=k(k+1) \cdots(k+n-1)$ is the Pochhammer symbol, and $\psi(k)$ being the Digamma function. Setting $n=2 k+1$ yields the result. Now, $\|u\|_{s, \gamma}=\inf \left\{\|U\|_{s+\frac{1}{2}, \gamma+\frac{1}{2}}|U|_{\partial \Omega}=u\right\}$, which leads to

$$
\left\|\gamma_{0}^{-} F g\right\|_{s+2 k, \gamma+2 k} \leq(2 k) !\|g\|_{s, \gamma} .
$$

On the other hand, we know that the extension operator $E: \mathcal{H}^{s, \gamma}(\partial \Omega) \rightarrow$ $\mathcal{H}^{s+\frac{1}{2}, \gamma+\frac{1}{2}}(\Omega)$ is continuous.

$$
\left\|\gamma_{0}^{-} F E g^{\prime}\right\|_{s+2 k, \gamma+2 k} \leq(2 k) !\left\|E g^{\prime}\right\|_{s, \gamma} \leq(2 k) !\left\|g^{\prime}\right\|_{s, \gamma} .
$$

for a $g^{\prime} \in \mathcal{H}^{s, \gamma}(\partial \Omega)$. But $\gamma_{0}^{-} F E g^{\prime}=\mathrm{T}_{\mathrm{log}, \mathrm{k}} g^{\prime}$.

\subsection{Example: Square as a Scattering Object}

In order to illustrate the parametrix construction we will study an example which is inspired by the triple junction graph in [OP04] in which the authors study a vertex of degree 3. In the case of a square which is a closed graph of degree 2 , all four angles $\alpha_{1}, \ldots, \alpha_{4}$ are equal to $\pi / 2$. This enables us to diagonalize the principle conormal symbol (interior symbols are unnecessary) and it therefore yields a simple expression. An obstacle shaped like a square enables us to diagonalize the principle conormal symbol since both $\alpha=\pi / 2$ and $\pi-\alpha=\pi / 2$.

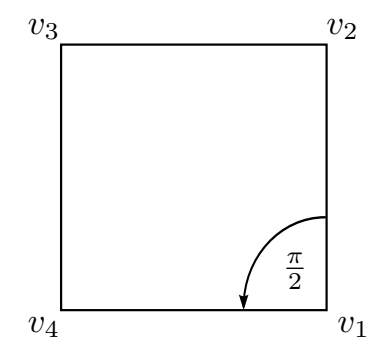

Figure 7.1: An obstacle shaped as a square 
Since all the four edges are straight lines, we may set the interior operators to zero and distribute their contributions to the Mellin operators. Consider the leading operators at the vertex $v_{j}$ :

$$
\mathcal{H}^{s, \gamma}\left(\partial \Omega_{j+1}\right) \oplus \mathcal{H}^{s, \gamma}\left(\partial \Omega_{j}\right) \rightarrow \mathcal{H}^{s+1, \gamma+1}\left(\partial \Omega_{j+1}\right) \oplus \mathcal{H}^{s+1, \gamma+1}\left(\partial \Omega_{j}\right)
$$

In the particular case of a rectangular obstacle, a square like in our example, we may diagonalize the above mapping by using a transformation of the form

$$
\hat{U}_{v_{j}}:\left[\begin{array}{c}
f_{j+1} \\
f_{j}
\end{array}\right] \mapsto \frac{1}{\sqrt{2}}\left[\begin{array}{c}
f_{j+1}-i f_{j} \\
f_{j+1}+i f_{j}
\end{array}\right] .
$$

Notice that we are making use of the identification $\phi \mathcal{H}^{s, \gamma}\left(\partial \Omega_{j}\right) \cong \phi \mathcal{H}^{s, \gamma}\left(\mathbb{R}_{+}\right)$ for a cut-off function $\phi$, and pay attention to the compatibility conditions. This results into the diagonal matrix

$$
\left[\begin{array}{cc}
\hat{\phi}_{j}^{j+1, \prime} \mathrm{T}_{0} \hat{\phi}_{j}^{j+1}+\tilde{\hat{\phi}}_{j}^{j+1} \mathrm{~T}_{0} \hat{\phi}_{j}^{j} & 0 \\
0 & i\left[\hat{\phi}_{j}^{j,} \mathrm{~T}_{0} \hat{\phi}_{j}^{j}-\tilde{\hat{\phi}}_{j}^{j} \mathrm{~T}_{0} \hat{\phi}_{j}^{j+1}\right]
\end{array}\right] .
$$

The conormal symbol is then given by (using $\alpha_{j}=\pi / 2, j=1,2,3,4$ ):

$$
\sigma_{\mathrm{M}, \mathrm{j}}^{-1}\left(\mathrm{~T}_{0}\right)(z)=\frac{\pi}{z-1} \tau^{-1}\left[\begin{array}{cc}
\frac{\cos (\pi z)+\cos \left(\frac{\pi}{2} z\right)}{\sin (\pi z)} & 0 \\
0 & i \frac{\cos (\pi z)-\cos \left(\frac{3}{2} \pi z\right)}{\sin (\pi z)}
\end{array}\right]
$$

Notice that $\tau^{-1}$ denotes the shift to the right by 1 in the complex plane.

$$
\sigma_{\mathrm{M}, \mathrm{j}}^{-1}\left(\mathrm{~T}_{0}\right)(z)=\frac{2 \pi}{z-1} \tau^{-1}\left[\begin{array}{cc}
\frac{\cos \left(\frac{3 \pi}{4} z\right) \cos \left(\frac{\pi}{4} z\right)}{\sin (\pi z)} & 0 \\
0 & i \frac{\sin \left(\frac{5 \pi}{4} z\right) \sin \left(\frac{\pi}{4} z\right)}{\sin (\pi z)}
\end{array}\right]
$$

The symbol is elliptic for weight $\gamma \notin \mathbb{Z}$. Now set

$$
\hat{G}(z):=\left[\begin{array}{cc}
\frac{\cos \left(\frac{3 \pi}{4} z\right) \cos \left(\frac{\pi}{4} z\right)}{\sin (\pi z)} & 0 \\
0 & i \frac{\sin \left(\frac{5 \pi}{4} z\right) \sin \left(\frac{\pi}{4} z\right)}{\sin (\pi z)}
\end{array}\right] .
$$

Notice that the nominators have roots in . We define the parametrix by setting $q(z):=\frac{1}{2 \pi} z G(z)^{-1}$ and

$$
\mathrm{Q}:=\sum_{j=1}^{4} \hat{\phi}_{j}^{\prime} t \mathrm{op}_{\mathrm{M}}^{\gamma}\left[\hat{q}_{j}\right] \hat{\phi}_{j} \in \mathcal{C}^{1}(\partial \Omega, \mathbf{g})
$$

with $\mathbf{g}=(1, \gamma, \Theta), \Theta=(-N, 0]$. 


\subsection{Proof of the Inequality}

Theorem 7.4 (Main theorem). There is a constant $C>0$ such that

$$
N_{m}^{ \pm}(r) \leq C r^{2}, \quad m \in \mathbb{Z}
$$

The proof of the main theorem consists of a series of lemmas which culminate in the following result:

Lemma 7.5. There exists a holomorphic function on the Riemann surface of the logarithm $d: \Lambda \rightarrow \mathbb{C}$ which satisfies the two properties:

1. The zeros of $d$ coincide with the poles of the scattering matrix both in location and multiplicity. I.e. if $\lambda_{j}$ is a scattering pole of multiplicity $k \geq 0$, then $d(\lambda)=0$ with the same multiplicity and vice versa.

2. $|d(\lambda)| \leq C e^{c|\lambda|^{2}}, \lambda \in \Lambda_{m}^{ \pm}$for each $m \in \mathbb{Z}$.

Applying this inequality to Jensen's formula (Theorem A.2.1) proves the main theorem. The first step is to apply Weyl's convexity estimate to the operator $\mathbb{1}+\mathscr{R}(\lambda)$ :

$$
|d(\lambda)| \leq \prod_{j=1}^{\infty}\left(1+\chi_{j}(\mathscr{R}(\lambda))\right)
$$

Then, we have to estimate the individual factors on the right hand side, i.e. $\chi_{j}(\mathscr{R}(\lambda)), j \in \mathbb{N}$. This is done by using the inequalities (A.7) and (A.8):

$$
\chi_{j}(\mathscr{R}(\lambda)) \leq \chi_{j}\left(\mathrm{Q} \mathrm{T}_{\log }(\lambda)\right)+\chi_{j}\left(\mathrm{QT}_{\mathrm{reg}}(\lambda)\right)
$$

The two terms on the right hand side are estimated by

$$
\chi_{j}\left(\mathrm{Q} \mathrm{T}_{\log }(\lambda)\right) \leq \sum_{k \geq 1} \frac{1}{(k !)^{2}}\left(\frac{\lambda}{2}\right)^{2 k} \chi_{j}\left(\mathrm{Q}_{\log , k}\right)
$$

and

$$
\chi_{j}\left(\mathrm{QT}_{\mathrm{reg}}(\lambda)\right) \leq A_{j}+B_{j}+C_{j}
$$

where

$$
\begin{aligned}
A_{j} & :=\kappa \sum_{k \geq 1} \frac{1}{(k !)^{2}}\left(\frac{\lambda}{2}\right)^{2 k} \chi_{j}\left(\mathrm{QT}_{\mathrm{reg}, k}\right), \\
B_{j} & :=-\frac{1}{2} \sum_{k \geq 1} a_{k} \frac{1}{(k !)^{2}}\left(\frac{\lambda}{2}\right)^{2 k} \chi_{j}\left(\mathrm{Q} \mathrm{T}_{\mathrm{reg}, k}\right), \\
C_{j} & :=\ln (\lambda) \sum_{k \geq 1} \frac{1}{(k !)^{2}}\left(\frac{\lambda}{2}\right)^{2 k} \chi_{j}\left(\mathrm{QT}_{\mathrm{reg}, k}\right) .
\end{aligned}
$$

It is therefore of crucial importance to estimate the numbers $\chi_{j}\left(\mathrm{QT}_{\log , k}\right)$ and $\chi_{j}\left(\mathrm{Q} \mathrm{T}_{\mathrm{reg}, k}\right)$ for $j \geq 1$. 
Theorem 7.6. The singular values satisfy the following estimate:

$$
\begin{aligned}
& \chi_{j}\left(\mathrm{QT}_{\log , k}\right) \leq c^{2 k}, \\
& \chi_{j}\left(\mathrm{QT}_{\log , k}\right) \leq c^{2 k}(2 k) ! j^{-2 k}
\end{aligned}
$$

for a constant $c>0$.

$$
\begin{aligned}
& \chi_{j}\left(\mathrm{QT}_{\mathrm{reg}, k}\right) \leq c^{2 k}, \\
& \chi_{j}\left(\mathrm{QT}_{\mathrm{reg}, k}\right) \leq c^{2 k}(2 k) ! j^{-2 k} .
\end{aligned}
$$

The proof of this assertion is based on Carl's inequality (Theorem A.5.1). Before we can continue with the proof, we first need to take a glance at the definition of entropy numbers:

Definition 7.7. Let $T: A \rightarrow B$ be a compact, linear operator between two complex Banach spaces $A$ and $B$. Let $\mathbb{B}_{A} \subset A$ and $\mathbb{B}_{B} \subset B$ be the unit balls, respectively. Then we define the $j^{\text {th }}$ entropy number of $T, j \in \mathbb{N}_{0}$, as

$$
\epsilon_{j}(T):=\inf \left\{\epsilon>0 \mid T\left(\mathbb{B}_{A}\right) \subseteq \bigcup_{m=0}^{2^{j-1}}\left(p_{m}+\epsilon \mathbb{B}_{B}\right), p_{j} \in B\right\} .
$$

Definition 7.8. Denote the compact embeddings between Sobolev spaces on $\partial \Omega$ for $s^{\prime}>s \geq 0$ by

$$
\iota_{s}^{s^{\prime}}: H^{s^{\prime}}(\partial \Omega) \hookrightarrow H^{s}(\partial \Omega) .
$$

Lemma 7.9. There exists a constants $c>0$, independent of the positive integers $j$ and $k$, such that

$$
\epsilon_{j}\left(\nu_{0}^{2 k}\right) \leq c j^{-2 k}
$$

for all $j, k \in \mathbb{N}_{0}$.

Since for $f \in H^{2 k}(\partial \Omega)$ the restrictions $f_{j}=\left.f\right|_{\partial \Omega_{j}}$ satisfy the compatibility conditions (3.9) at the vertices $v_{j}$, we may identify $f$ with a Sobolev function in $H^{2 k}(I), I \subset \mathbb{R}$ a suitable interval. The proof follows the arguments of [ET96, Theorem 3.3.2], but is very long and technical. One can verify that the constant $c>0$ is independent of $s^{\prime}>0$.

Remark 7.10. It is clear that the above argument is a rather weak link. However, a formal analysis of Edmund's and Triebel's proof requires more effort beyond of which we are capable in this thesis.

Remark 7.11. Notice that there is a lift operator $l_{2}: H^{s+2}(\partial \Omega) \rightarrow H^{s}(\partial \Omega)$ on the scale of Sobolev spaces. Although its usefulness has diminished during the course of this thesis, its proof of existence is shown in Lemma A.6.1 nonetheless.

Proof of Theorem 7.6. The inequalities without factorials are standard $L^{2}$ estimates. We therefore focus on the other two ones. Since, for $k \in \mathbb{N}_{0}, \mathrm{QT}_{k, \log }$ : $H^{s}(\partial \Omega) \rightarrow H^{s+2 k}(\partial \Omega)$, considered as $H^{s}(\partial \Omega) \rightarrow H^{s}(\partial \Omega)$, is a compact operator, we may apply Carl's inequality (Theorem A.5.1). That is, for $j \in \mathbb{N}_{0}$ we may estimate the $j^{\text {th }}$ entropy number as:

$$
\chi_{j}\left(\mathrm{QT}_{k, \log }\right) \leq \sqrt{2} \cdot \epsilon_{j}\left(\nu_{0}^{2 k}\right)\left\|\mathrm{Q} \mathrm{T}_{k, \log }\right\|_{0,2 k} .
$$


Due to Lemma 7.9, we know that

$$
\epsilon_{j}\left(\iota_{0}^{2 k}\right) \leq c j^{-2 k} .
$$

By Proposition 7.3, we have

$$
\left\|\mathrm{QT}_{\log , k}\right\|_{s, s+2 k} \leq(2 k) ! .
$$

The estimate (7.6) follows immediately.

Going back to the estimate (??), our next goal is to find an upper estimate for the singular numbers $\chi_{j}(\mathscr{R}(\lambda)), j \in \mathbb{N}_{0}$ :

Lemma 7.12. There exists a constant $C>0$ such that either

$$
\chi_{j}(\mathscr{R}(\lambda)) \leq C e^{C|\lambda|^{2}} \quad \text { for } j<C|\lambda|,
$$

or

$$
\chi_{j}(\mathscr{R}(\lambda)) \leq C|\lambda|^{2} j^{-1} \quad \text { for } j>C|\lambda| .
$$

Proof. Using both inequalities (A.7) and (A.8) yields

$$
\chi_{j}(\mathscr{R}(\lambda)) \leq \sum_{k \geq 1} \frac{1}{(k !)^{2}}\left(\frac{|\lambda|}{2}\right)^{2 k} \chi_{j}\left(\mathrm{QT}_{\log , k}\right)+A_{j}+B_{j}+C_{j},
$$

where $A_{j}, B_{j}$, and $C_{j}$ have been defined above. We only consider the first term with the sum.

Case $\mathbf{j} \leq \mathbf{c}|\lambda|$ : Using Theorem 7.6, inequality (7.6),

$$
\begin{aligned}
\sum_{k \geq 1} \frac{1}{(k !)^{2}}\left(\frac{|\lambda|}{2}\right)^{2 k} c^{2 k} & =\sum_{k \geq 1} \frac{1}{k !}\left(\frac{|\lambda|}{2}\right)^{2 k} c^{2 k} \\
& \leq \sum_{k \geq 1} \frac{1}{k !}\left(c|\lambda|^{2}\right)^{k} \\
& \leq e^{c|\lambda|^{2}} .
\end{aligned}
$$

Case $\mathbf{j}>\mathbf{c}|\lambda|$ : We make use of and the geometric series. Using the estimate (7.7) yields

$$
\sum_{k \geq 1} \frac{1}{(k !)^{2}}\left(\frac{|\lambda|}{2}\right)^{2 k} \chi_{j}\left(\mathrm{QT}_{\log , k}\right) \leq \sum_{k \geq 1} \frac{(2 k) !}{2^{2 k}(k !)^{2}}|\lambda|^{2 k} c^{2 k} j^{-2 k} .
$$

One can easily see that $2^{2 k}(k !)^{2} \geq(2 k)$ !. Hence, we can conclude

$$
\sum_{k \geq 1} \frac{1}{(k !)^{2}}\left(\frac{|\lambda|}{2}\right)^{2 k} \chi_{j}\left(\mathrm{QT}_{\log , k}\right) \leq \sum_{k \geq 1}\left(\frac{c|\lambda|}{j}\right)^{2 k} .
$$

Due to the condition $j>c|\lambda|$, this series is bounded by twice the first term. Estimating the other terms is done analogously.

Having proven the necessary lemmas, we may proceed to prove the main inequality: 
Proof. Now, our function is given by

$$
d(\lambda)=\operatorname{det}(\mathbb{1}+\mathscr{R}(\lambda)) .
$$

Weyl's convexity estimate (Theorem A.4.3) yields:

$$
\begin{aligned}
\left|d_{\mathscr{R}}(\lambda)\right| & \leq \prod_{j=1}^{\infty}\left(1+\chi_{j}(\mathscr{R}(\lambda))\right) \\
& =\underbrace{\prod_{j<C|\lambda|}\left(1+\chi_{j}(\mathscr{R}(\lambda))\right)}_{\text {I }} \underbrace{\prod_{j>C|\lambda|}\left(1+\chi_{j}(\mathscr{R}(\lambda))\right)}_{\text {II }} .
\end{aligned}
$$

Using Lemma ??, we may estimate the first factor, I, by inequality (??), i.e.

$$
\prod_{j<C|\lambda|}\left(1+\chi_{j}(\mathscr{R}(\lambda))\right) \leq \prod_{j<C|\lambda|}\left(1+C e^{C|\lambda|^{2}}\right) \leq C^{\prime} e^{C|\lambda|^{2}},
$$

and the second factor, II, by inequality (??):

$$
\prod_{j>C|\lambda|}\left(1+\chi_{j}(\mathscr{R}(\lambda))\right) \leq \prod_{j>C|\lambda|}\left(1+\frac{C|\lambda|^{2}}{j}\right)<\infty .
$$

Combining these two yields our sought-after result, namely

$$
|d(\lambda)| \leq C^{\prime \prime} e^{C|\lambda|^{2}}, \lambda \in \Lambda
$$

We are now in a position to apply Jensen's formula (Theorem A.2.1) and finalize the proof of the main theorem:

Proof of the main theorem. Inserting (7.13) into Jensen's formula, equation (A.6), yields

$$
N_{0}^{+}(r) \leq \frac{1}{\pi} \int_{0}^{\pi} \ln \left|d\left(r e^{i \theta}\right)\right| d \theta-\ln |d(0)| .
$$

Now with $\left|d\left(r e^{i \theta}\right)\right| \leq C e^{C r^{2}}$ and $|d(0)|=C$, we can calculate

$$
N_{0}^{+}(r) \leq C^{\prime} r^{2},
$$

which is the desired upper estimate. 


\section{Chapter 8}

\section{Outlook: Scattering Theory in Three Dimensions}

As we have indicated in the introduction, the scattering theory for the three dimensional problem is simpler, the elliptic theory more involved. The scattering theory can be followed along the lines of the monograph [LP67] with some easy modifications due to the non-smooth nature of the obstacle [LP78].

For the elliptic theory, consider only a single corner: Let $X$ be the base manifold. Since we are dealing with a polyhedron, $X$ decomposes into $N$ one dimensional segments $X=X_{1} \cup X_{2} \cup \ldots \cup X_{N}$ (A cube would only have a base manfold consiting of three segments, for example). In the corner, we would have to deal with operator-valued symbols of the form

$$
z \mapsto \sum_{j=1}^{N} \int_{X_{j}} a(\xi, \eta ; z) f_{j}(\eta) d s_{j}(\eta)
$$

where $\xi, \eta \in X, f_{j} \in \mathcal{H}^{s, \gamma}\left(X_{j}\right)$, and $d s$ is the surface measure on the cone base $X$.

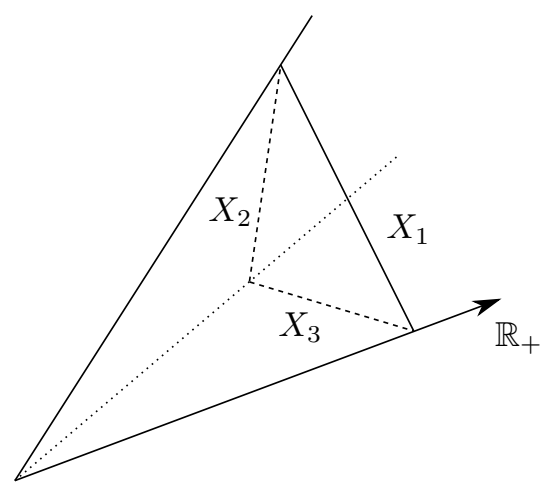

Figure 8.1: Corner of a polyhedral obstacle in three dimensions 
The fundamental solution of wave equation in three dimensions is given by

$$
r_{0}(x, y ; \lambda)=\frac{1}{4 \pi} \frac{e^{i \lambda|x-y|}}{|x-y|} \text { for } x \neq y, \text { and } \lambda \in \mathbb{C} .
$$

The term of leading order as $x \rightarrow y$ of course

$$
a_{1}(x)=\frac{1}{4 \pi} \frac{1}{|x|}, \quad x \in \mathbb{R}^{3} \backslash\{0\} .
$$

It is well-known [Sch89] that the Mellin transform of the fundamental solution of the Laplacian given by

$$
z \mapsto \frac{1}{4 \sin (\pi z)} P_{z-1}(-\xi \cdot \eta), \quad \xi, \eta \in X
$$

where $P_{\mu}=P_{\mu}^{0}$ denotes the Legendre function of first kind and (complex) degree $\mu \in \mathbb{C}:{ }^{1}$

$$
P_{z-1 / 2}(\cos \theta)=\frac{2}{\pi} \int_{0}^{\theta}(2 \cos s-2 \cos \theta)^{-\frac{1}{2}} \cos (s z) d s
$$

One can see that for $\xi \rightarrow \eta$ and $z \in \mathbb{C}$ fixed, that

$$
P_{z-1}(-\xi \cdot \eta) \sim-\ln |\varphi-\psi|+\ldots
$$

This is indeed the qualitative behaviour which we are expecting.

One of the complexities in three dimensions, aside of higher notational efforts, is that we have to deal with the edges. That is, we would need to consider integral operators of the form

$$
\mathbb{R} \ni \sigma \mapsto \int_{\mathbb{R}_{+}} \frac{1}{\sqrt{\sigma^{2}+\left(r-r^{\prime}\right)^{2}}} f\left(r^{\prime}\right) d r^{\prime},
$$

where $f \in C_{c}^{\infty}\left(\mathbb{R}_{+}\right)$, where $\sigma \in \mathbb{R}$ parametrized the edge. A brute force approach by calculating the Mellin transform of the kernel does not seem to be fruitful, so the question arises whether one should continue to proceed via calculations or via abstract theory.

The formulation of the three dimensional theory would almost certainly involve elements of edge calculus [Sch98].

Other points of concern are the modifications of the jump relations. We have seen in Theorem 6.10 that the jump relations for the double layer potential in two dimensions have to be modified for the singular case. Are there analogous modifications in the three dimensional case? This will certainly be the case, but this time we will need to modify the relations in the corners of the polyhedron as well as along the edges. The modifications along an edge $E$ would perhaps be of the form $\alpha_{E}(\sigma) / \pi$, where $\alpha_{E}(\sigma)$ is the (interior) contact angle between the two faces meeting in $E$, and $\sigma$ is again a parametrization of the edge. The form of the modifying factor in a corner $V$ will look different from the two dimensional case and is a matter of calculating the appropriate asymptotics. All in all, the three dimensional problem seems to be a challenging problem.

${ }^{1}$ Legendre functions are a generalization of Legendre polynomials 


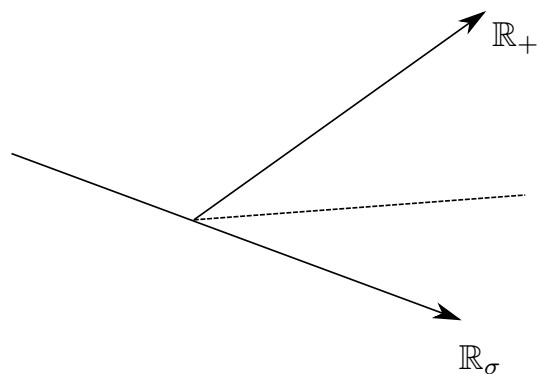

Figure 8.2: Edge of a polyhedral obstacle in three dimensions 


\section{Appendix}

\section{A.1 Singularities in Boundary Value Problems}

\section{A.1.1 Green's Identity}

This subsection contains definitions and results for elliptic boundary value problems in a more general context. They can all be found in [Dau88].

Let $\Omega \subset \mathbb{R}^{n}$ be a polyhedral domain.

Definition A.1.1. A d-face of $\Omega, d \in\{1, \ldots, n\}, \partial^{d, i} \Omega, i=1, \ldots, I_{d}$ denoted by $\partial^{\prime} \Omega, n_{x}=d$. Each $\partial^{d, i} \Omega$ is a domain in a $n-d$ dimensional smooth manifold $M^{d, i}$. The set $\partial^{d, i} \Omega$ is called the canonical set of faces of $\Omega$.

Definition A.1.2. An admissible set of faces $\left\{\partial^{d, i} \Omega \mid d, i\right\}$ is a partition of $\partial \Omega$ such that

1. $\partial^{d, i} \Omega$ is a Lipshitz polyhedral domain in a smooth $(n-d)$ dimensional manifold $M^{d, i}$.

2. For each $x \in \partial^{d^{\prime}, i^{\prime}} \Omega, d^{\prime} \geq 2$, there is a smooth chart $\chi_{x}$ sending a neighborhood of $x$ in $\bar{\Omega}$ onto a neighborhood of 0 in a cone $\overline{C_{x}}$, and, for any $(d, i)$ such that $x \in \overline{\partial \Omega}$ sending a neighborhood of $x$ in onto a neighborhood of 0 in a polyhedral cone.

For an admissible set $\left\{\partial^{d, i} \Omega\right\}$ of $\Omega$. In each point $x \in \overline{\partial^{d, i} \Omega},(d, i)$ fixed, there are local coordinates $(\nu, \tau)$ such that

$$
\begin{array}{cl}
\nu=\left(\nu_{1}, \ldots, \nu_{d}\right) & \text { are normal to } M^{(d, i)}, \\
\tau=\left(\tau_{1}, \ldots, \tau_{n-d}\right) & \text { are tangential to } M^{(d, i)} .
\end{array}
$$

Denote the normal derivatives by $\partial_{\nu}$ and the tangential derivatives by $\partial_{\tau}$.

Definition A.1.3. The family $\left\{B^{d, i, \alpha}\left(x, \partial_{x}\right)\left|\alpha \in \mathbb{N}^{d},\right| \alpha \mid \leq l\right\}$ is called a $l$-order normal system of $n^{\text {th }}$ order on $\partial^{d, i} \Omega$, if

1. $B^{d, i, \alpha}$ are operators with order $|\alpha|$ and smooth coefficients on a neighborhood of $\partial^{i, d} \Omega$ in $M^{i, d}$.

2. For each $x \in \overline{\partial^{d, i} \Omega}$ and each $j=0, \ldots, l$, the principle parts of $\left\{B^{d, i, \alpha}\left(x_{0}, \partial_{\nu}, 0\right) \mid\right.$ $|\alpha|=j\}$ form a basis of the homogeneous degree $j$ operators with constant coefficients on $\mathbb{R}^{n}$, and, for $(d, i)$ such that $x \in \overline{\partial^{d, i} \Omega}$, sending a neighborhood of $x$ in $\overline{\partial^{d, i} \Omega}$ onto a neighborhood of 0 in a polyhedral cone $\overline{\partial^{d, i} C_{x}} \in \mathcal{C}_{n-d}$. The $\partial^{d, i} C_{x}$ are a partition of the boundary of $C_{x}$. 
For $l=1$, this is the usual definition of a normal system.

Theorem A.1.4. Let $\Omega$ be a polyhedral domain and $\left\{\partial^{d, i} \Omega\right\}$ an admissible set of faces. Let $m \in \mathbb{N}$. For each $(d, i)$, let $\left\{B^{d, i, \alpha} \mid \alpha\right\}$ be a $(m-d)$-order normal system on $\partial \Omega$. Let a be a integrodifferential form with $C^{\infty}(\bar{\Omega})$-coefficients and let $L$ be the $2 m$-order operator associated to $a$. Then there exists operators $C^{d, i, \alpha}$ with order $2 m-|\alpha|-d$ and $C^{\infty}\left(M^{d, i}\right)$ coefficients such that the following Green's formula holds for any $u \in H^{2 m}(\Omega)$ and $v \in H^{m}(\Omega)$ :

$$
a(u, v)=\int_{\Omega} L u \bar{v} d y+\sum_{1 \leq d \leq n} \sum_{1 \leq i \leq I_{d}} \sum_{|\alpha| \leq m-d} \int_{\partial^{d, i} \Omega} C^{d, i, \alpha} u B^{d, i, \alpha} \bar{v} d s(y)
$$

If $m-d<0$, the system is empty. If $d=n, \partial^{n, i} \Omega$ reduces to a point $x_{0}$ and

$$
\int_{\partial^{d, i} \Omega} f d y=f\left(x_{0}\right) \text {. }
$$

Corollary A.1.5. Consider the gradient form

$$
a(u, v)=\int_{\Omega} \nabla u \nabla \bar{v} d y
$$

As $m=1$, only the 1-faces can be equipped with a normal system with order 0 . The Green's formula may then be written as

$$
a(u, v)=-\int_{\Omega} \Delta u \bar{v} d y+\sum_{1 \leq i \leq 1} \int_{\partial^{1, i} \Omega} \partial_{\nu} u \bar{v} d s(y) .
$$

Corollary A.1.6.

$$
\int_{\Omega}[\Delta u \bar{v}-u \Delta \bar{v}] d y=\sum_{1 \leq i \leq I_{1}} \int_{\partial^{1, i} \Omega}\left[\partial_{\nu} u \bar{v}-u \partial_{\nu} \bar{v}\right] d s(y) .
$$

\section{A.1.2 Regularity of Solutions of Cone-Degenerate BVPs}

In contrast to the case of a domain $\Omega$ with smooth boundary, Poisson's equation

$$
\Delta u=f \in L^{2}(\Omega)
$$

with homogeneous Dirichlet boundary condition does not simply have a solution $u \in H^{2}(\Omega)$. Rather, the solution is a sum $u=u_{R}+u_{\text {sing }}$ of a regular term $u_{R} \in H^{2}(\Omega)$ and a singular term $u_{\text {sing }} \in H^{2-\delta}(\Omega), 0<\delta<2$. The latter consists of explicit terms which are localized in the vicinity of the domain's corners and have the form

$$
S_{j, 1}(r, \vartheta)=r^{\frac{\pi}{\alpha_{j}}} \sin \left(\pi \frac{\vartheta}{\alpha_{j}}\right),
$$

where $\alpha_{j}$ is the interior angle at the vertex $v_{j} .{ }^{2}$ Of course, the theory may be extended to different boundary conditions, i.e. homogeneous and inhomogeneous Dirichlet and Neumann conditions. Moreover, one may apply different boundary conditions to different edges. However, these considerations are not important for the problem and their further study is left to the reader [Gri92]. Summarizing this discussion, we obtain the following result.

\footnotetext{
${ }^{2}$ Note, that we are considering the Laplace equation in the interior of the domain at the moment, whereas our scattering problem deals with the exterior of the domain.
} 
Theorem A.1.7. Consider the Poisson equation (A.4) on a polygonal domain with straight edges and homogeneous Dirichlet boundary equations. Then there exists an unique solution $u \in H^{2-\delta}(\Omega), \delta=\max \left\{\pi / \alpha_{j} \mid j=1, \ldots, N\right\}$ of the problem. The solution is of the form $\lambda_{j, m}=m \pi / \alpha_{j}$.

$$
u=u_{R}+\sum_{j=1}^{N}\left(\sum_{0<\lambda_{j, m}<1} c_{j, m} S_{j, m}\right),
$$

where $u_{R} \in H^{2}(\Omega)$ is the regular part, and $c_{j, m} \in \mathbb{C}$.

\section{A.2 Jensen's Formula}

Jensen's formula relates the number of zeros of an analytic function $h$ to its growth properties:

Theorem A.2.1 (Jensen's formula). Let $h$ be a analytic function in a region in the complex plane which contains the closed disk $\mathbb{D}_{r}(0)$ of radius $r>0$ about the origin. Let $\left\{z_{k}\right\}_{k=1}^{N}$ be the zeros of $h$ in the interior of $\mathbb{D}_{r}(0)$, counted according to their multiplicity. Then Jensen's formula states

$$
\log |h(0)|=\sum_{k=1}^{N} \log \left(\frac{\left|z_{k}\right|}{r}\right)+\frac{1}{2 \pi} \int_{0}^{2 \pi} \log \left|h\left(r e^{i \theta}\right)\right| d \theta .
$$

Corollary A.2.2. Let $N_{h}(r)$ be the number of zeros of $h$ in the interior of the disc $\mathbb{D}_{r}(0)$. Then this number can be estimated by

$$
N_{h}(r) \leq \frac{1}{2 \pi \log (2)} \int_{0}^{2 \pi} \log \frac{\left|h\left(2 r e^{i \theta}\right)\right|}{|h(0)|} d \theta .
$$

Proof. Of the $N_{h}(r)$ zeros in $\mathbb{D}_{r}(0)$,consider only the first $N_{h}(r / 2)$ zeros which are contained in the interior of $\mathbb{D}_{\frac{r}{2}}(0)$ and satisfy

$$
\left|z_{k}\right|<\frac{r}{2}, \quad k=1, \ldots, N_{h}(r / 2)
$$

This means we can estimate

$$
\log 2 \leq \log \left(\frac{r}{\left|z_{k}\right|}\right), \quad k=1, \ldots, N_{h}(r / 2),
$$

and for the rest of the elements in the annulus $\mathbb{D}_{r}(0) \backslash \mathbb{D}_{\frac{r}{2}}(0)$ :

$$
0 \leq \log \left(\frac{r}{\left|z_{k}\right|}\right), \quad k=N_{h}(r / 2)+1, \ldots, N_{h}(r),
$$

This again implies

$$
N_{h}(r / 2) \cdot \log (2) \leq \sum_{k=1}^{N_{h}(r / 2)} \log (2) \leq \sum_{k=1}^{N_{h}(r)} \log \left(\frac{r}{\left|z_{k}\right|}\right) .
$$

Inserting the integral, we have

$$
N_{h}(r / 2) \leq \frac{1}{2 \pi \log (2)} \int_{0}^{2 \pi} \log \frac{\left|h\left(r e^{i \theta}\right)\right|}{|h(0)|} d \theta .
$$

Substituting $r$ with twice the radius, i.e. $2 r$, yields the desired result. 


\section{A.3 Analytic Fredholm Theorem}

Theorem A.3.1. Let $D \subset \mathbb{C}$ be a connected open subset, $G(z)$ a compact, operator-valued, holomorphic function of $z \in D$. Suppose that $\mathbb{1}+G(z)$ is invertible at some point $z_{0} \in D$. Then $\mathbb{1}+G(z)$ is invertible everywhere on $D$ except at most for a discrete set $D \subset U$, and $(\mathbb{1}+G(z))^{-1}$ is meromorphic on $U$.

\section{A.4 Trace Class Operators}

Let $\mathcal{H}$ be a Hilbert space, $T: \mathcal{H} \rightarrow \mathcal{H}$ a compact operator. Denote the eigenvalues of $T$ by $\lambda_{k}(T)$. If $T$ is self-adjoint, then the operator has a complete set of eigenvalues with only zero as accumulation point. If $T$ is not self-adjoint, then we may consider $|T|:=\sqrt{T^{*} T}, T^{*}$ being the adjoint of $T$.

Definition A.4.1 (Singular values). The singular values of the operator $T$ are eigenvalues its modulus, $|T|$, ordered in decreasing order. I.e., we have $\chi_{k}(T):=\lambda_{k}(|T|)$ for all $k \in \mathbb{N}$ and

$$
\chi_{1}(A) \geq \chi_{2}(A) \geq \ldots \rightarrow 0
$$

Definition A.4.2 (Fredholm determinant). Let $T$ be of trace class. We define the Fredholm determinant $\operatorname{det}(\mathbb{1}+T)$ by

$$
\operatorname{det}(\mathbb{1}+T):=\prod_{k=1}^{\infty}\left(1+\lambda_{k}(T)\right) .
$$

The product is convergent.

Theorem A.4.3 (Weyl's convexity estimate). Let $T$ be an operator of trace class.

$$
|\operatorname{det}(\mathbb{1}+T)| \leq \prod_{j=1}^{\infty}\left(1+\chi_{j}(T)\right)=\operatorname{det}(1+|T|) \leq e^{t r|T|}
$$

Theorem A.4.4. Let $S: \mathcal{H} \rightarrow \mathcal{H}$ be bounded.

$$
\chi_{j}(S T) \leq\|S\| \chi_{j}(B)
$$

If $S$ is compact, then

$$
\begin{aligned}
\chi_{i+j-1}(S+T) & \leq \chi_{i}(S)+\chi_{j}(T), \\
\chi_{i+j}(S T) & \leq \chi_{i}(S) \chi_{j}(T) .
\end{aligned}
$$

\section{A.5 Carl's Inequality}

Theorem A.5.1 (Carl's inequality). Let $T: A \rightarrow A$ be a compact linear operator in a complex Banach space $A$. Let $\left(\chi_{k}(T)\right)_{k \in \mathbb{N}}$ be the sequence of eigenvalues of $|T|$ as above. Then:

$$
\chi_{k}(T) \leq \sqrt{2} e_{k}(T) \quad \text { for all } k \in \mathbb{N} .
$$


Corollary A.5.2. Let $S: H^{s}(\Omega) \rightarrow H^{s^{\prime}}(\Omega)$ be a bounded linear operator, $s>s^{\prime}, \Omega \subset \mathbb{R}^{n}$ a domain. Denote $k^{\text {th }}$ entropy number of the compact embedding $\iota_{s}^{s^{\prime}}: H^{s^{\prime}}(\Omega) \rightarrow H^{s}(\Omega)$ by $\epsilon_{k}\left(\iota_{s}^{s^{\prime}}\right)$. Let $S^{\prime}:=\iota_{s}^{s^{\prime}} S$ with singular values $\left(\chi_{k}\left(S^{\prime}\right)\right)_{k \in \mathbb{N}}$ as above. Then

$$
\chi_{k}\left(S^{\prime}\right) \leq \sqrt{2} \epsilon_{k}\left(\iota_{s}^{s^{\prime}}\right)\|S\|_{s, s^{\prime}} \quad \text { for all } k \in \mathbb{N},
$$

where $\|-\|_{s, s^{\prime}}$ denotes the operator norm in $L\left(H^{s}(\Omega), H^{s^{\prime}}(\Omega)\right)$.

\section{A.6 A Technical Lemma}

Lemma A.6.1. Consider the scale of Sobolev spaces $H^{s}(\partial \Omega), s \geq 0$. The operator

$$
T=\left(\mathbb{1}-\partial_{\tau}^{2}\right): H^{s+2}(\partial \Omega) \rightarrow H^{s}(\partial \Omega)
$$

is a positive and self-adjoint isomorphism with $\|T f\|_{s} \leq 2\|f\|_{s+2}$.

Proof. Consider the operator $-\Delta_{\partial \Omega}$ on $\partial \Omega$. On $\partial \Omega_{j}$, assuming parametrization by arc length, we have $-\Delta_{\Gamma}=-\partial_{t}^{2}$. Consider the maximal operator $T=1-\Delta_{\partial \Omega}$ with maximal domain $\mathcal{D}\left(\mathrm{T}_{\max }\right)=\prod_{j=1}^{N} H^{2}\left(\partial \Omega_{j}\right)$, and set

$$
w(f, g, t):=\operatorname{det}\left[\begin{array}{cc}
f(t) & g(t) \\
\partial_{\tau} f(t) & \partial_{\tau} g(t)
\end{array}\right], \quad t \in \partial \Omega_{j} .
$$

Recall that the scalar product on $\partial \Omega$ is given by

$$
\langle f, g\rangle_{\partial \Omega}:=\sum_{j=1}^{N} \int_{\partial \Omega_{j}} \overline{f_{j}}(t) g_{j}(t) d s_{j}(t) .
$$

Now consider

$$
\begin{aligned}
\sum_{j=1}^{N}\left(\left\langle f_{j}, \partial_{\tau}^{2} g_{j}\right\rangle_{\partial \Omega_{j}}-\left\langle\partial_{\tau}^{2} f_{j}, g_{j}\right\rangle_{\partial \Omega_{j}}\right) & =\sum_{j=1}^{N}\left(w\left(\overline{f_{j}}, g_{j}, l_{j}\right)-w\left(\overline{f_{j}}, g_{j}, 0\right)\right) \\
& =\sum_{j=1}^{N}\left(w\left(\overline{f_{j-1}}, g_{j-1}, l_{j-1}\right)-w\left(\overline{f_{j}}, g_{j}, 0\right)\right),
\end{aligned}
$$

where we have rearranged the sum in the last line. We need to choose adequate boundary conditions at the endpoints of the intervals in order to define a selfadjoint operator. The minimal choice would be to demand $f_{j}, g_{j} \in H_{0}^{2}\left(\partial \Omega^{1, j}\right)$ for all $j$. One other possible choice is to set

$$
\left[\begin{array}{c}
f_{j-1}\left(l_{j-1}\right) \\
\partial_{\tau} f_{j-1}\left(l_{j-1}\right)
\end{array}\right]=\alpha\left[\begin{array}{c}
f_{j}(0) \\
\partial_{\tau} f_{j}(0)
\end{array}\right], \quad \alpha \in \mathbb{C} \text { with }|\alpha|=1 .
$$

This choice leads the column vectors of the Wronski determinant to be linearly dependent such that and the right hand side of (A.10) will vanish. Since we demand compatibility conditions, set $\alpha=1 . T$ is therefore symmetric. 
That $T$ is strictly positive follows immediately by partial integration,

$$
\langle f, T f\rangle=\sum_{j=1}^{N} \int_{0}^{l_{j}}\left(\left|\partial_{\tau} f_{j}(t)\right|^{2}+\left|f_{j}(t)\right|^{2}\right) d s_{j}(t)>0
$$

for any non-zero $f \in \mathcal{D}(T)$. The injectivity of $T$ follows immediately from its positivity. The estimate can be seen by a calculation with respect to the norm

$$
\|f\|_{m}=\sum_{j=0}^{N} \sum_{k=0}^{m} \int_{\partial \Omega_{j}}\left|\partial_{\tau}^{k} f(t)\right|^{2} d t, \quad m \in \mathbb{N}_{0},
$$

and a subsequent estimate. Again, complex interpolation yields the result. 


\section{Acknowledgements}

First and foremost, I'd like to thank the Research Training Group 1493 Mathematical Structures in Modern Quantum Physics for its financial support by their scholarship program. Its trust and confidence in me was very surprising, and I truly hope that I was able to keep up with the high expectations imposed on me. I still hope that I will prove myself worthy in the years after this thesis, either by my own contributions or by inspiring a new generation, in the very least case friends, family, and, one day, perhaps my own children.

I'd like to thank my thesis advisor, Prof. Ingo Witt, for his patience and for supervising my work.

I am very indebted to Prof. Dorothea Bahns for her kind support during the late stages of this thesis. She contributed both by giving me the confidence to continue my work and asking intelligent and useful questions.

Special thanks also goes to Professors Dorothee Haroske and Hans Triebel for their extensive efforts in trying to teach me the theory of entropy numbers.

I'd also like to say thanks to the members of our working group, but especially to Huong and Thang for their kind hospitality when cooking Vietnamese cuisine, and Xiaowen and Jiguang for our numerous discussions in the offices about the differences and similarities between the Chinese and Japanese languages.

Many thanks also go to my mother and my brother Markus who always supported my drive for knowledge and offered support whenever I was starting to loose my faith. Thank you, Elena, for your encouragement and love. 


\section{Bibliography}

[Bar99] Antônio Sá Barreto. "Lower bounds for the number of resonances in even dimensional potential scattering". In: Journal of Functional Analysis 169.1 (1999), pp. 314-323.

[CH05] Tanya Christiansen and Peter D. Hislop. "The resonance counting function for Schrödinger operators with generic potentials". In: Math. Research Letters 12.6 (2005), pp. 821-826.

[CH10] T.J. Christiansen and P.D. Hislop. "Maximal Order of Growth for the Resonance Counting Functions for Generic Potentials in Even Dimensions". In: Indiana Univ. Math. J. 59.2 (2010), pp. 621-660.

[CH14a] T.J. Christiansen and P. Hislop. "Lower bounds for resonance counting functions for obstacle scattering in even dimensions". In: arXiv preprint math-ph/1409.7608 (2014).

[CH14b] T.J. Christiansen and P.D. Hislop. "Some remarks on resonances in even-dimensional euclidean scattering". In: Transactions of the American Mathematical Society 368.2 (2014), pp. 1361-1385.

[Chr02] Tanya Christiansen. "Some upper bounds on the number of resonances for manifolds with infinite cylindrical ends". In: Annales Henri Poincaré. Vol. 3. 5. Springer. 2002, pp. 895-920.

[Chr06] T.J. Christiansen. "Schrödinger operators with complex-valued potentials and no resonances". In: Duke Mathematical Journal 133.2 (2006), pp. 313-323.

[Chr15] T.J. Christiansen. "A sharp lower bound for a resonance-counting function in even dimensions". In: arXiv preprint math-ph/1510.04952 (2015).

[Dau88] Monique Dauge. Elliptic Boundary Value Problems in Corner Domains - Smoothness and Asymptotics of Solutions. Vol. 1341. Lecture Notes in Mathematics. Berlin: Springer, 1988.

[Dud99] Roland Duduchava. "The Green Formula and Layer Potentials". In: Integral Equations and Operator Theory 41.2 (June 1999), pp. 127178.

[EB97] Yuri V. Egorov and Schulze Bert-Wolfgang. Pseudo-Differential Operators, Singularities, Applications. Operator Theory, Advances and Applications, Vol. 93. Basel: Birkhäuser, 1997.

[ET96] D. E. Edmunds and Hans Triebel. Function Space, Entropy Number, and Differential Operators. Cambridge University Press, 1996. 
[GR07] I.S. Gradshteyn and I.M. Ryzhik. Table of integrals, series, and products. Translated from the Russian. English. 7th ed. Amsterdam: Elsevier/Academic Press, 2007.

[Gri85] Pierre Grisvard. Elliptic Problems in Nonsmooth Domains. Pitman Publishing, 1985.

[Gri92] Pierre Grisvard. Singularities in Boundary Value Problems. Recherches en mathématiques appliquées. Masson, 1992.

[Gru09] Gerd Grubb. Distributions and operators. Springer, 2009.

[His12] Peter D Hislop. "Fundamentals of scattering theory and resonances in quantum mechanics". en. In: Cubo (Temuco) 14 (Oct. 2012), pp. 01-39.

[Int86] Ahmed Intissar. "A polynomial bound on the number of the scattering poles for a potential in even dimensional spaces $\mathbb{R}^{n}$." English. In: Commun. Partial Differ. Equations 11 (1986), pp. 367-396.

[Kre99] Rainer Kress. Linear integral equations. Applied mathematical sciences. New York: Springer, 1999.

[LP64] Peter D. Lax and Ralph S. Phillips. "Scattering theory". In: Bull. Amer. Math. Soc. 70 (1 1964), pp. 130-142.

[LP67] Peter D. Lax and Ralph S. Phillips. Scattering Theory. New York: Academic Press, 1967.

[LP72] Peter D. Lax and Ralph S. Phillips. "Scattering Theory for the Acoustic Equation in an Even Number of Space Dimensions". In: Indiana Univ. Math. J. 22 (1972), pp. 101-134.

[LP78] Peter D. Lax and Ralph S. Phillips. "Scattering Theory for Domains with Non-Smooth Boundaries". In: Arch. Rational. Mech. Anal. 68 (2 1978), pp. 93-98.

[Mel83] Richard Melrose. "Polynomial bounds on the number of scattering poles". In: J. Funct. Anal. 53 (1983), pp. 287-303.

[Mel84] Richard B. Melrose. "Polynomial bound on the distribution of poles in scattering by an obstacle". eng. In: Journées équations aux dérivées partielles (1984), pp. 1-8.

[Olv74] F. W. J. Olver. Asymptotics and Special Functions. Academic Press, 1974.

[OP04] Petri Ola and Lassi Päivärinta. "Mellin operators and pseudodifferential operators on graphs". In: Waves in Random Media 14.1 (2004), S129-S142.

[RS78] Michael Reed and Barry Simon. Methods of modern mathematical physics. III. , Scattering Theory. London: Academic Press, 1978.

[RS89] S Rempel and BW Schulze. Asymptotics for Eliptic Mixed Boundary Problems. Akademie-Verlag, 1989.

[Sch89] Hermann Schmitz. "Über das Singuläre Verhalten der Lösungen von Integralgleichungen auf Flächen mit Ecken". dissertation. Universität Stuttgart, 1989. 
[Sch91] B-W Schulze. Pseudo-differential operators on manifolds with singularities. Elsevier, 1991.

[Sch95] Bert-Wolfgang Schulze. Pseudo-differential boundary value problems, conical singularities, and asymptotics. Berlin: Akademie Verlag, 1995.

[Sch98] B.-W. Schulze. Boundary Value Problens and Singular Peudo-differential Operators. Wiley Ser. Pure Appl. Math. J. Wiley, 1998.

[SS94] Elmar Schrohe and Bert-Wolfgang Schulze. "Boundary Value Problems in Boutet de Monvel's algebra for manifolds with conical singularities I". In: Boundary Value Problems, Schrödinger Operators, Deformation Quantization. Advances in Partial Differential Equations. Akademie Verlag, 1994, pp. 97-209.

[SS95] Elmar Schrohe and Bert-Wolfgang Schulze. "Boundary Value Problems in Boutet de Monvel's algebra for manifolds with conical singularities II". In: Pseudo-Differential Calculus and Mathematical Physics. Advances in Partial Differential Equations. Akademie Verlag, 1995, pp. 70-205.

[Tay13] M. Taylor. Partial Differential Equations II: Qualitative Studies of Linear Equations. Applied Mathematical Sciences. Springer New York, 2013.

[Vod91a] Georgi Vodev. "Polynomial bounds on the number of scattering poles for metric perturbations of the Laplacian in $\mathbb{R}^{n}, n \geq 3$, odd". In: Osaka. J. Math. 28 (1991), pp. 441-449.

[Vod91b] Georgi Vodev. "Sharp polynomial bounds on the number of scattering poles for metric perturbations of the Laplacian in $\mathbb{R}^{n}$ ". English. In: Mathematische Annalen 291.1 (1991), pp. 39-49.

[Vod92] Georgi Vodev. "Sharp bounds on the number of scattering poles for perturbations of the Laplacian". In: Comm. Math. Phys. 146.1 (1992), pp. 205-216.

[Wat66] G.N. Watson. Treatise on the Theory of Bessel Functions. Cambridge University Press, 1966.

[Wil75] Calvin H. Wilcox. Scattering Theory for the d'Alembert Equation in Exterior Domains. Lecture Notes in Mathematics, No. 442. Berlin: Springer-Verlag, 1975.

[Zwo89] Maciej Zworski. "Sharp polynomial bounds on the number of scattering poles". In: Duke Math. J. 59.2 (Oct. 1989), pp. 311-323.

[Zwo94] M. Zworski. "Counting scattering poles". In: Spectral and scattering theory. Lectures in Pure and Appl. Math. 161. New York: Dekker, 1994, pp. 301-331. 